\title{
Extremal black holes, nilpotent orbits and the true fake superpotential
}

\author{
Guillaume Bossard, ${ }^{a}$ Yann Michel $^{b}$ and Boris Pioline ${ }^{b}$ \\ ${ }^{a}$ AEI, Max-Planck-Institut für Gravitationsphysik, \\ Am Mühlenberg 1, D-14476 Potsdam, Germany \\ ${ }^{b}$ Laboratoire de Physique Théorique et Hautes Energies, ${ }^{1}$ \\ Université Pierre et Marie Curie - Paris 6, \\ 4 place Jussieu, F-75252 Paris cedex 05, France \\ E-mail: bossard@aei.mpg.de, ymichelpro@gmail.com, \\ pioline@lpthe.jussieu.fr
}

ABSTRACT: Dimensional reduction along time offers a powerful way to study stationary solutions of 4D symmetric supergravity models via group-theoretical methods. We apply this approach systematically to extremal, BPS and non-BPS, spherically symmetric black holes, and obtain their "fake superpotential" $W$. The latter provides first order equations for the radial problem, governs the mass and entropy formula and gives the semi-classical approximation to the radial wave function. To achieve this goal, we note that the Noether charge for the radial evolution must lie in a certain Lagrangian submanifold of a nilpotent orbit of the 3D continuous duality group, and construct a suitable parametrization of this Lagrangian. For general non-BPS extremal black holes in $\mathcal{N}=8$ supergravity, $W$ is obtained by solving a non-standard diagonalization problem, which reduces to a sextic polynomial in $W^{2}$ whose coefficients are $\mathrm{SU}(8)$ invariant functions of the central charges. By consistent truncation we obtain $W$ for other supergravity models with a symmetric moduli space. In particular, for the one-modulus $S^{3}$ model, $W^{2}$ is given explicitely as the root of a cubic polynomial. The STU model is investigated in detail and the nilpotency of the Noether charge is checked on explicit solutions.

KeYwords: Supersymmetry and Duality, Black Holes in String Theory, Black Holes

ARXIV EPRINT: 0908.1742

\footnotetext{
${ }^{1}$ Unité mixte de recherche du CNRS UMR 7589
} 


\section{Contents}

1 Introduction $\quad 2$

1.1 Radial evolution and geodesic motion 2

1.2 Extremal solutions and fake superpotential 4

1.3 Fake superpotential and radial wave function 5

$\begin{array}{ll}1.4 & \text { Extremal black holes and nilpotent orbits }\end{array}$

$\begin{array}{lll}1.5 & \text { Strategy and main results } & 7\end{array}$

$\begin{array}{llr}1.6 & \text { Outline } & 9\end{array}$

2 Extremal black holes in $\mathcal{N}=8$ supergravity 9

2.1 Generalities on $\mathcal{N}=8$ supergravity $\quad 9$

2.2 Spherically symmetric, weakly extremal solutions 11

$\begin{array}{lll}2.3 & 1 / 8-\text { BPS black holes } & 14\end{array}$

$\begin{array}{lll}2.4 & \text { Non-BPS extremal black holes } & 18\end{array}$

3 Truncations of maximal supergravity and their extensions $\quad 24$

$\begin{array}{ll}3.1 \text { Magic } \mathcal{N}=2 \text { supergravity } & 25\end{array}$

$\begin{array}{ll}3.2 & \text { Axion-dilaton } \mathcal{N}=2 \text { supergravity }\end{array}$

$\begin{array}{lll}3.3 \mathcal{N}=4 \text { supergravity } & 31\end{array}$

3.4 Axion-dilaton gravity $\quad 32$

4 Extremal black holes in the $S T U$ model $\quad 34$

4.1 Moduli spaces in $D=4$ and $D=3 \quad 34$

$\begin{array}{lll}4.2 & \text { Near horizon solutions } & 40\end{array}$

4.3 Extremal $Z_{*}=0$ solutions $\quad 41$

4.4 Extremal $Z_{*} \neq 0$ solutions 43

$\begin{array}{ll}\text { A Nilpotent orbits } & 44\end{array}$

A.1 Generalities 44

A.2 Nilpotent orbits of $E_{8(8)} \quad 46$

A.3 Nilpotent orbits of $\mathrm{SO}(4,4) \quad 50$

B Solving the non-BPS diagonalization problem $\quad 54$

B.1 General case $\quad 54$

B.2 Near $\varphi=\pi / 4 \quad 57$

B.3 At $\rho_{2}=\rho_{3}-S^{2} T$ model $\quad 58$

B.4 At $\rho_{1}=\rho_{2}=\rho_{3}-S^{3}$ model $\quad 58$

B.5 Near $\varphi=0 \quad 60$ 
$\begin{array}{lr}\text { C Explicit solutions } & 60\end{array}$

$\begin{array}{lll}\text { C.1 D0-D4, BPS } & 60\end{array}$

C.2 D0-D4, non-BPS $Z_{*}=0 \quad 61$

$\begin{array}{lll}\text { C.3 } & \text { D0-D4, non-BPS } Z \neq 0 & 62\end{array}$

\section{Introduction}

In trying to extend our string-theoretic understanding of black holes away from the supersymmetric regime, extremality is often a key simplifying assumption. Firstly, it eliminates Hawking radiation and ensures that the solution is semi-classically stable. Secondly, it implies the existence of an AdS region which may admit a dual conformal field theory description. Thirdly, it guarantees that the near-horizon solution is entirely determined by the conserved charges measurable at spatial infinity, and therefore insensitive (away from lines of marginal stability) to variations to the moduli at infinity. This attractor behavior, first discovered for supersymmetric (BPS) black holes [1, 2], holds for all extremal solutions [3-5], and is arguably responsible for the validity of certain weakly coupled description of non-BPS black hole micro-states [6].

However, while the most general BPS solution is known explicitly [7, 8], our ability to construct non-BPS extremal solutions is quite limited. Early solutions were found in $[9,10]$ by an astute embedding of the Reissner-Nordström solution in $\mathcal{N}=8$ supergravity, while some solutions were studied numerically in [11]. More recently, it was shown how to deduce first-order equations for non-BPS extremal solutions from a "fake superpotential" [12-15], and some solutions were obtained. Unfortunately, this strategy has suffered from the lack of a general method to construct the fake superpotential. A generic 5-parameter seed solution was obtained and analyzed in [14, 16-19]. Using dimensional reduction along the time direction, non-BPS solutions of the one-modulus $\mathcal{N}=2$ supergravity model were obtained in [20] via the determination of their nilpotent Noether charge. The nilpotent orbits $^{1}$ associated to extremal black holes have been constructed in [25, 26].

In this paper, we build upon the insights of $[12,20,25]$ and give a systematic method to construct the fake superpotential for non-BPS extremal solutions in supergravity models with a symmetric moduli space. In particular, we derive the fake superpotential for extremal non-BPS black holes in $\mathcal{N}=8$ supergravity in full generality, and in magic $\mathcal{N}=2$ supergravity models by consistent truncation. Before giving the outline of the paper, we start by briefly reviewing the dimensional reduction, fake superpotential and nilpotent orbit techniques which underlie our approach.

\subsection{Radial evolution and geodesic motion}

Stationary solutions in $D=4$ supergravity are efficiently studied by reduction along the time direction [21, 27-29] (see e.g. [30] for a review). After dualizing the one-forms in three

\footnotetext{
${ }^{1}$ The relation between extremal black holes and nilpotent orbits was first uncovered in the BPS case in [21], generalized to 5D black holes in [22], and has been further developed since in [23, 24].
} 
dimensions and restricting to weakly extremal ${ }^{2}$ solutions, one obtains a non-linear sigma model with pseudo-Riemannian target space

$$
\mathcal{M}_{3}^{*} \sim \mathbb{R}^{+} \times \mathcal{M}_{4} \times T \times S^{1},
$$

where

- $\mathbb{R}^{+}$is parametrized by the scale function $U$ in the metric ansatz

$$
d s^{2}=-e^{2 U}(d t+\omega)^{2}+e^{-2 U}\left(d r^{2}+r^{2}\left(d \theta^{2}+\sin ^{2} \theta d \phi^{2}\right)\right)
$$

- $\mathcal{M}_{4}$ is the moduli space of massless scalar fields in 4 dimensions, with coordinates $\phi^{i}$ and metric $g_{i j}$

- $T$ is a $2 n_{V}$ - dimensional symplectic torus parametrized by the Wilson lines $\zeta^{\Lambda}, \tilde{\zeta}_{\Lambda}$ of $A^{\Lambda}$ and its magnetic dual $A_{\Lambda}$ around the time direction, and

- $S^{1}$ is the fibre of a circle bundle over $T$ parametrized by the NUT potential $\sigma$ dual to the off-diagonal metric one-form $\omega$. Its first Chern class is proportional to the canonical symplectic form $d \zeta^{\Lambda} \wedge d \tilde{\zeta}_{\Lambda}$.

We shall denote the coordinates $U, \phi^{i}, \zeta^{\Lambda}, \tilde{\zeta}_{\Lambda}, \sigma$ on $\mathcal{M}_{3}^{*}$ collectively as $\phi^{\mu}$, and $U, \phi^{i}$ as $\phi^{a}$. As indicated by the $\sim$ sign, the metric on $T \times S^{1}$ varies over the base $\mathbb{R}^{+} \times \mathcal{M}_{4},{ }^{3}$ being positive definite along $S^{1}$ and negative definite along $T$, while the metric on the base is $\frac{1}{2} d U^{2}+g_{i j} d \phi^{i} d \phi^{j} \equiv g_{a b} d \phi^{a} d \phi^{b}$, positive definite. The negative signature along $T$ can be traced to the negative signature of the time direction along which the dimensional reduction is carried out. In the context of $\mathcal{N}=2$ supergravity, $\mathcal{M}_{4}$ is special Kähler, and $\mathcal{M}_{3}^{*}$, the "c*-map" of $\mathcal{M}_{4}$, is related to the "c-map" of [31] by analytic continuation $(\zeta, \tilde{\zeta}, \sigma) \mapsto$ $(i \zeta, i \tilde{\zeta},-\sigma)$. Under this dimensional reduction, stationary solutions of $4 \mathrm{D}$ supergravity become harmonic maps from $\mathbb{R}^{3}$ to $\mathcal{M}_{3}^{*}$, with pointwise vanishing Lagrangian density (this latter condition follows from the restriction to weakly extremal solutions).

Assuming in addition spherical symmetry, the supergravity equations of motion become equivalent to light-like geodesic motion on $\mathcal{M}_{3}^{*}$, with the affine parameter $\tau$ identified as the inverse radial distance $\tau=1 / r$. The conserved Noether charges along the twisted torus $T \times S^{1}$ are identified as the electric, magnetic and NUT charges $q_{\Lambda}, p^{\Lambda}, k$, respectively. Static solutions have zero NUT charge $k=0$. In this case the Hamiltonian for light-like geodesic motion on $\mathcal{M}_{3}^{*}$ becomes independent of $\zeta^{\Lambda}, \tilde{\zeta}_{\Lambda}$, and reduces to the Hamiltonian for the motion of a fiducial particle on $\mathbb{R}^{+} \times \mathcal{M}_{4}$ subject to the potential $V$,

$$
H=\frac{1}{2} p_{a} g^{a b} p_{b}+V\left(p, q ; \phi^{a}\right) \equiv 0 .
$$

\footnotetext{
${ }^{2}$ We define weak extremality as the condition that the three-dimensional spacial slices be flat. In order to be extremal, such a solution must also be smooth.

${ }^{3}$ Whereas this fibration over $\mathbb{R}^{+} \times \mathcal{M}_{4}$ is globally defined on the Riemannian target space $\mathcal{M}_{3}$ which appears in the space-like reduction, it only holds on a dense open set of $\mathcal{M}_{3}^{*}$ homeomorphic to $\mathcal{M}_{3}$. Nevertheless, the complement of this open set has support at $U=-\infty$, and this subtlety appears to be irrelevant for the purposes of this paper.
} 
Here $p_{a}=g_{a b} \dot{\phi}^{b}$ is the momentum conjugate to $\phi^{a}$, the dot denotes the derivative with respect to $\tau$, and $V \equiv-e^{2 U} V_{\mathrm{BH}}$ encodes the (negative definite) kinetic energy along $T$. The latter depends quadratically on the charges $p, q$, and is proportional to $V_{\mathrm{BH}}$, which is sometimes called the "black hole potential".

\subsection{Extremal solutions and fake superpotential}

While the condition that the spatial slices be flat is necessary for extremality, it is by no means sufficient. In order that the solution be smooth, one must fine-tune the boundary conditions at spatial infinity so that the particle reaches the top of the potential hill in infinite proper time and with zero velocity, $p_{a}(\tau=\infty)=0$. For fixed electromagnetic charges, this fine-tuning holds only on a Lagrangian subspace of the phase space $\left(\phi^{a}, p_{a}\right)$ of initial conditions at $\tau=0$.

There can be different ways of performing this fine-tuning. In supergravity models with extended supersymmetry, one may impose the existence of Killing spinors to obtain first order equations which relate the momentum $p_{a}$ to the coordinate $\phi^{a}$, and guarantee that the second order equations of motion are obeyed. The resulting solution then preserves some fraction of supersymmetry and, if smooth, will also be extremal. This is most familiar in the framework of $D=4, \mathcal{N}=2$ supergravity, where BPS black holes satisfy the "attractor flow" equations

$$
p_{a}=-\partial_{\phi^{a}} \mathcal{S}_{\mathrm{BPS}},
$$

i.e. follow the gradient flow of the potential

$$
\mathcal{S}_{\mathrm{BPS}}\left(\phi^{a}\right)=e^{U} W_{\mathrm{BPS}}, \quad W_{\mathrm{BPS}}=\left|Z_{p, q}\left(\phi^{i}\right)\right| .
$$

The potential $\mathcal{S}_{\mathrm{BPS}}$ and "superpotential" $W_{\mathrm{BPS}}$ satisfy

$$
V=-g^{a b} \partial_{a} \mathcal{S}_{\mathrm{BPS}} \partial_{b} \mathcal{S}_{\mathrm{BPS}}=-e^{2 U}\left(W_{\mathrm{BPS}}^{2}+2 g^{i j} \partial_{i} W_{\mathrm{BPS}} \partial_{j} W_{\mathrm{BPS}}\right)
$$

such that on solutions of (1.4), the positive kinetic energy compensates the potential term $V\left(\phi_{a}\right)<0$, ensuring that the spatial slices $d s_{3}^{2}$ are flat. Moreover, they guarantee that a maximum of $V$ (or minimum of $V_{\mathrm{BH}}$ ) is reached at zero momentum, provided this extremum occurs at a regular attractor point $\left|Z_{*}\right|>0$ [32]. The restriction of (1.4) at $\tau=0$ define the "BPS" component of the Lagrangian subspace of extremal solutions, and corresponds to BPS black holes with ADM mass and Bekenstein-Hawking entropy given by

$$
2 G M=W_{\mathrm{BPS}}(\tau=0), \quad S_{\mathrm{BH}}=\pi W_{\mathrm{BPS}}^{2}(\tau=\infty) .
$$

There may however exist other disconnected components of the Lagrangian subspace of extremal solutions corresponding to non-BPS black holes. As shown in [12, 13], some non-BPS solutions can be obtained in a similar way as the BPS ones, provided there exists another function $\mathcal{S}\left(\phi^{a}\right)=e^{U} W\left(\phi^{i}\right)$, where $W\left(\phi^{i}\right)$ is dubbed the "fake superpotential", such that the potential $V$ can be written as in (1.6),

$$
V=-e^{2 U}\left(W^{2}+2 g^{i j} \partial_{i} W \partial_{j} W\right)=-g^{a b} \partial_{a} \mathcal{S} \partial_{b} \mathcal{S} .
$$


The first order equations

$$
p_{a}=-\partial_{\phi^{a}} \mathcal{S}\left(\phi^{a}\right),
$$

then imply, just as in the BPS case, that the second order equations of motion are satisfied, that the kinetic and potential energy compensate each other, and that the solutions reach a critical point of the potential at zero velocity. The first order equations (1.9) at $\tau=0$ therefore provide another component of the Lagrangian subspace of extremal solutions, and correspond to extremal non-BPS black holes with mass and Bekenstein-Hawking entropy given by (1.7) where $W_{\mathrm{BPS}}$ is replaced by $W$. In contrast to the BPS case, the critical point of $W$ is not guaranteed to be an isolated maximum, but could exhibit flat directions or even saddle behavior; in the presence of flat directions, some of the scalars at the horizon are determined uniquely by the conserved charges, although the entropy will be independent of the asymptotic value of the scalars [4]. Using this method, non-BPS extremal black holes for the $S T U$ model were obtained in [12] in the axion-free case. ${ }^{4}$ Unfortunately, there has been no systematic way of computing $W$ without solving for the full problem (although, in some cases, one may engineer different fake superpotentials $W$ for the same potential $V$ using discrete symmetries). The purpose of this paper is to give a method to determine $W$ a priori for symmetric supergravity models.

\subsection{Fake superpotential and radial wave function}

As a side remark, we note that the potential $\mathcal{S}\left(\phi^{a}\right)$ may be identified, by virtue of (1.9), as the generating function of the Lagrangian subspace of the "small phase space" $T_{*}\left(\mathbb{R}^{+} \times \mathcal{M}_{4}\right)$ corresponding to smooth extremal solutions with fixed values of the electromagnetic charges (see [34] for a related discussion). By construction, $\mathcal{S}$ solves the Hamilton-Jacobi equation ${ }^{5}$ associated to the Hamiltonian (4.13)

$$
H\left(\partial_{\phi^{a}} \mathcal{S}, \phi^{a}\right)=\partial_{t} \mathcal{S}=0
$$

Equivalently, both the first order equations (1.9) and the relations ${ }^{6}$

$$
p^{\Lambda}=-\frac{\sqrt{2}}{2}\left(p_{\tilde{\zeta}_{\Lambda}}-\zeta^{\Lambda} p_{\sigma}\right), \quad q_{\Lambda}=-\frac{\sqrt{2}}{2}\left(p_{\zeta^{\Lambda}}+\tilde{\zeta}_{\Lambda} p_{\sigma}\right), \quad k=-2 p_{\sigma}
$$

between the charges $q_{\Lambda}, p^{\Lambda}, k$ and the canonical momenta $p_{\zeta^{\Lambda}}, p_{\tilde{\zeta}_{\Lambda}}, p_{\sigma}$ can be derived from the generating function on the "large phase space" $T_{*}\left(\mathcal{M}_{3}^{*}\right)$

$$
\tilde{\mathcal{S}}\left(\phi^{\mu}\right)=-4 \mathcal{S}\left(\phi^{a}\right)+\sqrt{2}\left(q_{\Lambda} \zeta^{\Lambda}+p^{\Lambda} \tilde{\zeta}_{\Lambda}\right)
$$

\footnotetext{
${ }^{4} \mathrm{~A}$ fake superpotential for non-zero axion was postulated in [33], but it depends explicitely on the flat directions and its status is unclear to us.

${ }^{5}$ Hamilton's principle function is usually a function of the position variables at time $t$, canonical momenta at initial time 0 , and time $t$ itself. The electric and magnetic charges $p^{\Lambda}, q_{\Lambda}$ in (1.12) can be regarded as the values of the canonical momenta at $t=0$, while the absence of explicit time dependence is a consequence of weak extremality, $H=0$.

${ }^{6}$ The numerical factors in the forthcoming relations are convention-dependent, and have been chosen consistently with the coordinates used for the $S T U$ model in section 4.
} 
via $p_{\phi^{\mu}}=\partial_{\phi^{\mu}} \tilde{\mathcal{S}}$. Upon quantization of the radial evolution of the scalars by replacing $p_{i}=\frac{i}{\hbar} \partial_{\phi^{i}}[21,35]$, this generating function determines the semi-classical form of the radial wave function, i.e.

$$
\Psi\left(\phi^{a}\right) \sim \exp \left(\frac{i}{\hbar} \tilde{\mathcal{S}}\left(\phi^{a}\right)\right) \sim \exp \left(\frac{i}{\hbar} e^{U} W\left(\phi^{i}\right)+i \sqrt{2}\left(q_{\Lambda} \zeta^{\Lambda}+p^{\Lambda} \tilde{\zeta}_{\Lambda}\right)\right)
$$

For BPS black holes with superpotential (1.5), one recovers the semi-classical BPS wave function found in $[35,36]$. In addition to the usefulness of $W$ for determining the mass, entropy and fine-tuning at infinity, this relation to the radial wave function provides extra incentive to study the fake superpotential for non-BPS extremal black holes.

\subsection{Extremal black holes and nilpotent orbits}

In this note, we focus on the special case of $\mathcal{N} \geq 2$ supergravity theories with a symmetric moduli space, where group theoretical methods can be used to bear on this problem. In these cases, both $\mathcal{M}_{4}$ and $\mathcal{M}_{3}^{*}$ are symmetric spaces,

$$
\mathcal{M}_{4}=K_{4} \backslash G_{4}, \quad \mathcal{M}_{3}^{*}=K_{3}^{*} \backslash G_{3} .
$$

Here $K_{4}$ is the maximal compact subgroup of the continuous 4 D duality group $G_{4}$, while $K_{3}^{*}$ is a non-compact real form of the maximal compact subgroup of the continuous 3D duality group $G_{3}$. The latter acts isometrically on $\mathcal{M}_{3}^{*}$ by right-multiplication, and yields a conserved Noether charge $Q$ valued in the Lie algebra ${ }^{7} \mathfrak{g}_{3}$. Those include not only the conserved charges for translations along the twisted torus $T \times S_{1}$, i.e. the electromagnetic and NUT charges, but also additional charges corresponding to Ehlers and Harrison transformations, as well as $4 \mathrm{D}$ duality rotations. The geodesic motion on $\mathcal{M}_{3}^{*}$ is integrable, and in fact all geodesics on $\mathcal{M}_{3}^{*}$ can be obtained by exponentiating a generator $-P_{0} \tau \in \mathfrak{g}_{3} \ominus \mathfrak{k}_{3}^{*}$, where $P_{0}$ determines the momentum along the trajectory. $P_{0}$ is conjugate to the Noether charge $Q$ via the coset representative $\mathcal{V}$ in $G_{3}$,

$$
P \equiv-\left.\left(\dot{\mathcal{V}} \mathcal{V}^{-1}\right)\right|_{\mathfrak{g} \ominus \mathfrak{k}^{*}}=\mathcal{V} Q \mathcal{V}^{-1}
$$

Extremal solutions correspond to special geodesics which reach the boundary $U=-\infty$ in infinite proper time [27]. As already mentioned, it is necessary but not sufficient that the geodesic be light-like. For BPS black holes, it was observed in [21] that the Noether charge must satisfy $[\operatorname{ad}(Q)]^{5}=0$, i.e. belong to a nilpotent orbit of degree 5 (See appendix A for a summary of useful facts about nilpotent orbits). More precisely, it was shown that the Noether charge defines a 5-grading ${ }^{8}$ of the Lie algebra $\mathfrak{g}_{3}=\mathfrak{g}^{(-4)} \oplus \mathfrak{g}^{(-2)} \oplus \mathfrak{g}^{(0)} \oplus \mathfrak{g}^{(2)} \oplus \mathfrak{g}^{(4)}$ where the top spaces $\mathfrak{g}^{(4)}$ is one-dimensional. Upon quantization, the BPS phase space becomes the Hilbert space of the quaternionic discrete series of $G_{3}$ [35], closely related to the quasiconformal realization [37].

\footnotetext{
${ }^{7} Q$ is more naturally valued in the dual Lie algebra $\mathfrak{g}_{3}^{*}$, but we can identify the two using the Killing form.

${ }^{8}$ To match standard conventions in the mathematics literature on nilpotent orbits, we rescale the Cartan generator $\mathbf{h}$ by a factor of 2 compared to [21], such that the 5-grading becomes an even 9-grading; the original 5 -grading with charges ranging from -2 to 2 corresponds to the minimal orbit.
} 
For what concerns extremal non-BPS black holes, it was later shown in the special case of the one-modulus $S^{3}$ model that extremality requires the condition $\left[Q_{\mid 7}\right]^{3}=0$, where 7 denotes the 7 -dimensional representation of the 3D duality group $G_{2(2)}[20]$. This condition is equivalent to $[\operatorname{ad}(Q)]^{5}=0$ in this particular case. $G_{2(2)}$ admits two distinct nilpotent orbits of degree 5 with the same dimension 10, corresponding to extremal BPS and non-BPS black holes, respectively.

More recently, the supersymmetry and extremality conditions on the Noether charge for symmetric supergravity models were re-analyzed in [24]. It was shown in all cases where $G_{3}$ is simple that extremality requires ${ }^{9}$ to $\left[Q_{\mid \mathbf{R}}\right]^{3}=0$, where $\mathbf{R}$ denotes the "fundamental representation" of $G_{3}$ : for example the spinor representation if $G_{3}$ is an orthogonal group $\mathrm{SO}(2+m, 2+n)$ or $S O^{*}(2 m+4)$. The only exception is for $G_{3}=E_{8(8)}$ or $E_{8(-24)}$, where the condition becomes $\left[Q_{\mid \mathbf{3 8 7 5}}\right]^{5}=0$, with $\mathbf{3 8 7 5}$ being the 3875 -dimensional irreducible representation appearing in the symmetric tensor product of two adjoints.

More precisely, any generic extremal spherically symmetric black hole (i.e. with a nonzero horizon area) is characterized by a nilpotent Noether charge $Q$ which lies inside the grade-two component $\mathfrak{r}_{4}^{(2)}$ of $\mathfrak{g}_{3}$ with respect to the 5 -grading (more appropriately, even 9 -grading) which arises in the reduction from 4 to 3 dimensions:

$$
\mathfrak{g}_{3} \cong \mathbf{1}^{(-4)} \oplus \mathfrak{r}_{4}^{(-2)} \oplus\left(\mathfrak{g l}_{1} \oplus \mathfrak{g}_{4}\right)^{(0)} \oplus \mathfrak{l}_{4}^{(2)} \oplus \mathbf{1}^{(4)}
$$

The nilpotent orbit $\mathcal{O}_{G_{3}}$ of $Q \in \mathfrak{g}_{3}$ under $G_{3}$ is characterized by the isotropy subgroup of $Q$ in $G_{4}$. For extremal black holes, this isotropy subgroup coincides with the isotropy subgroup of the electromagnetic charges in the four-dimensional duality group $G_{4}$ computed in $[38,39]$. On the other hand, the momentum $P_{0}$ is valued in the coset $\mathfrak{g}_{3} \ominus \mathfrak{k}_{3}^{*}$, and therefore defines a $K_{3}^{*}$-orbit $\mathcal{O}_{K_{3}^{*}}$ inside $\mathfrak{g}_{3} \ominus \mathfrak{k}_{3}^{*}$. As explained in [25], $\mathcal{O}_{K_{3}^{*}}$ is a Lagrangian submanifold of $\mathcal{O}_{G_{3}}$ equipped with its canonical Kirillov-Kostant symplectic form. Parametrizing this Lagrangian will be a key step towards computing the fake superpotential.

\subsection{Strategy and main results}

Since the coset component of the Maurer-Cartan form is conjugate to the Noether charge via $P=\mathcal{V} Q \mathcal{V}^{-1}$, it defines a representative $\mathbf{e} \equiv P$ of the corresponding nilpotent orbit inside the coset component $\mathfrak{g}_{3} \ominus \mathfrak{k}^{*}$, and therefore defines a $K^{*}$-orbit inside this coset. A general fact about nilpotent elements is that one can always find another nilpotent element $\mathbf{f}$ and a semi-simple generator $\mathbf{h}$ such that the triplet $(\mathbf{e}, \mathbf{f}, \mathbf{h})$ defines an $\mathfrak{s l}_{2}$ subalgebra of $\mathfrak{g}_{3}$, i.e.

$$
[\mathbf{e}, \mathbf{f}]=\mathbf{h}, \quad[\mathbf{h}, \mathbf{e}]=2 \mathbf{e}, \quad[\mathbf{h}, \mathbf{f}]=-2 \mathbf{f} .
$$

The eigenspaces of $\mathbf{h}$ furnish a graded decomposition of $\mathfrak{g}$ which uniquely characterizes the complex nilpotent $G_{\mathbb{C}}$ orbit [40]. Extremal solutions are such that the $K^{*}$-orbit of $P$ is characterized by a graded decomposition of $\mathfrak{k}^{*}$ of the same form as (1.16) [25],

$$
\mathfrak{k}^{*} \cong \mathfrak{k}^{(-4)} \oplus \mathfrak{k}^{(-2)} \oplus \mathfrak{g l}_{1} \oplus \mathfrak{k}^{(0)} \oplus \mathfrak{k}^{(2)} \oplus \mathfrak{k}^{(4)}
$$

\footnotetext{
${ }^{9} \mathrm{An}$ important assumption in [24] is that all extremal solutions can be obtained as limits of non-extremal black hole solutions. Irrespective of this, the condition $\left[Q_{\mid \mathbf{R}}\right]^{3}=0$ must be supplemented by a condition on $P$ as discussed at the end of section A.1.
} 
As we shall show explicitly in the framework of $\mathcal{N}=8$ supergravity, for static solutions (i.e. with zero NUT charge) the semi-simple element $\mathbf{h}$ associated to the nilpotent element $P$ can be computed in terms of the central charges $Z_{i j}$ alone, and more generally, in terms of the central and matter charges which we write collectively $Z_{I} .{ }^{10}$ Decomposing $P \in \mathfrak{g} \ominus \mathfrak{k}^{*}$ with respect to the Ehlers $\mathrm{U}(1)$ and the four-dimensional R-symmetry group $K_{4}$,

$$
P=-\dot{U} \mathbf{H}+e^{U} Z_{I} \mathbf{L}^{I}-e_{i}^{j} \dot{\phi}^{i} \mathbf{G}_{j} \in \mathbb{C} \oplus \mathfrak{l}_{4} \oplus\left(\mathfrak{g}_{4} \ominus \mathfrak{k}_{4}\right),
$$

where $e_{i}{ }^{j}$ is a vielbein for the metric $g_{i j}$, one may recast the middle equation in (1.17) into a system of first order differential equations of the form

$$
\dot{U}=-e^{U} W, \quad g_{i j} \dot{\phi}^{j}=-e^{U} W_{i},
$$

where $W$ and $W_{i}$ depend on the moduli $\phi^{i}$ and electromagnetic charges $Q_{I}$ through the charges $Z_{I}$ only; moreover, we shall prove that

$$
W_{i}=\partial_{\phi^{i}} W .
$$

Thus, extremal solutions attached to the given nilpotent orbit satisfy a gradient flow under the fake superpotential $W$. In particular, it follows from the nilpotency of $P$ that

$$
\operatorname{Tr} P^{2}=0=e^{2 U}\left(W^{2}-Z_{I} Z^{I}+2 g^{i j} W_{i} W_{j}\right)=0,
$$

and therefore that (1.8) is obeyed.

Applying this strategy to $\mathcal{N}=8$ supergravity with $G_{3}=E_{8(8)}$, we are able to determine the fake superpotentials for both BPS and non-BPS extremal black holes, and express them in terms of the $\mathrm{SU}(8)$ invariant combinations of the central charges:

- In the BPS case, we find that $W$ is the modulus of the largest skew eigenvalue of the central charge matrix (in particular, $W^{2}$ is largest root of a quartic polynomial whose coefficients are polynomials in $Z_{i j}$ ). This reproduces the result of [13].

- In the non-BPS case, we find that $W=2 \varrho$, where $\varrho$ is obtained from the non-standard diagonalization problem (2.68). This problem is solved in appendix $\mathrm{B}$, where $W^{2}$ is expressed as a particular root of an irreducible sextic polynomial. ${ }^{11}$ This polynomial becomes reducible at particular values of the central charges, at which points $W$ can be computed in closed form. In particular, on the semi-line $\operatorname{Pfaff}(Z) \in \mathbb{R}_{\text {- we recover }}$ the result of [13].

Our expression for the fake superpotential in fact extends straightforwardly to all theories with a symmetric scalar manifold whose isometry group acts faithfully on the electromagnetic charges [27], as discussed in section 3 below. In particular, we obtain the fake superpotential for all magic $\mathcal{N}=2$ supergravity models, and in fact for all supergravity theories with $\mathcal{N} \geq 2$ with a symmetric moduli space. In the one-modulus case, $W$ takes a completely explicit form given in (3.33) below.

\footnotetext{
${ }^{10}$ Here $Z_{I}$ are the scalar field dependent linear combinations of the electromagnetic charges, transforming in a complex representation of $K_{4}$ and such that $V_{\mathrm{BH}}=Z_{I} Z^{I}$.

${ }^{11} \mathrm{By}$ irreducible, we mean as a polynomial with coefficients defined as rational functions of the $\mathrm{SU}(8)$ invariant polynomials in $Z_{i j}$, or more formally, within the field extension of $\mathbb{Q}$ generated by these invariants.
} 


\subsection{Outline}

In section 2, we apply the above strategy and find the complete fake superpotential for BPS and non-BPS extremal black holes in $\mathcal{N}=8$ supergravity. In section 3 , we extend these results to $\mathcal{N}=4$ and symmetric $\mathcal{N}=2$ supergravity models. In section 4 , we analyze the $S T U$ model in more detail, rephrase the BPS and non-BPS, $Z_{*}=0$ solutions in terms of the para-quaternionic geometry of $\mathcal{M}_{3}^{*}$, and check the nilpotency of the Noether charge on explicit solutions. Appendix A contains a detailed discussion of the real nilpotent orbits of $E_{8(8)}$ and $\mathrm{SO}(4,4)$, relevant for maximal supergravity and the STU model. In appendix B we discuss how to evaluate the fake superpotential for non-BPS, $Z \neq 0$ black holes at various loci in the space of central charges corresponding to consistent truncations. Appendix C records some extremal solutions of the STU model, which provide a useful testing bench for our analysis.

Note added: the fake superpotential (3.33) for the $S^{3}$ model was derived independently in [57], which appeared on arXiv after the present work had been completed.

\section{Extremal black holes in $\mathcal{N}=8$ supergravity}

In this section, we parametrize the $\operatorname{Spin}^{*}(16)$ orbits of generic extremal spherically symmetric black holes of $\mathcal{N}=8$ supergravity, as Lagrangian submanifolds of certain nilpotent orbits of $E_{8(8)}$ in $\mathfrak{e}_{8(8)} \ominus \mathfrak{s o}^{*}(16)$. For static solutions, this parametrization determines $P$ in terms of the central charges and allows us to identify the fake superpotential. We reproduce the known result for the fake superpotential for 1/8-BPS black holes (2.42), and obtain a new expression (2.78) for the fake superpotential for non-BPS extremal black holes, valid everywhere in moduli space.

\subsection{Generalities on $\mathcal{N}=8$ supergravity}

We first set up our notations for $\mathcal{N}=8$ supergravity in 4 dimensions. The massless scalar fields take values in the symmetric space [42]

$$
\mathcal{M}_{4} \cong \mathrm{SU} \backslash E_{7(7)},
$$

where $\mathrm{SU}$ is the quotient of $\mathrm{SU}(8)$ by the $\mathbb{Z}_{2}$ centre leaving invariant the representations of even rank. According to the conventions of [43] (up to normalization factors), we write the coset representative $v$ as

$$
v \hat{=}\left(\begin{array}{cc}
u_{i j}{ }^{I J} & v_{i j K L} \\
v^{k l I J} & u^{k l} K L
\end{array}\right),
$$

where little Latin letters are associated to the $\mathrm{SU}(8)$ gauge symmetry, whereas capital Latin letters refer to the global SU $\subset E_{7(7)}$. They both run from 1 to 8 , and raising or lowering indices corresponds to complex conjugation (e.g. $\Phi^{I J}=\left(\Phi_{I J}\right)^{*}$ and $\left.Z^{i j}=\left(Z_{i j}\right)^{*}\right)$. The invariant metric on $\mathcal{M}_{4}$ can be written as

$$
d s_{\mathcal{M}_{4}}^{2}=\frac{1}{24} V_{i j k l} V^{i j k l}
$$


where

$$
V_{i j k l}=u_{i j}^{I J} d v_{k l I J}-v_{i j I J} d u_{k l}^{I J}
$$

is the $\mathrm{SU} \backslash E_{7(7)}$ vielbein, which is automatically a complex self-dual antisymmetric tensor by property of the $\mathfrak{e}_{7(7)}$ Lie algebra. In the symmetric gauge, $v$ can be written in terms of a complex self-dual tensor $\phi_{i j k l}=\frac{1}{24} \varepsilon_{i j k l m n p q} \phi^{m n p q}$,

$$
v \hat{=} \exp \left(\begin{array}{cc}
0 & \phi_{i j k l} \\
\phi^{i j k l} & 0
\end{array}\right)=\left(\begin{array}{ll}
\cosh (\phi)_{i j}{ }^{k l} & \sinh (\phi)_{i j k l} \\
\overline{\sinh }(\phi)^{i j k l} & \overline{\cosh }(\phi)^{i j}{ }_{k l}
\end{array}\right)
$$

The hyperbolic functions in this expression are Taylor series in $\phi_{i j k l}$ with $\mathrm{SU}(8)$ covariant contractions [43],

$$
\begin{aligned}
\cosh (\phi)_{i j}{ }^{k l} & =\delta_{i j}^{k l}+\frac{1}{2} \phi_{i j p q} \phi^{p q k l}+\mathcal{O}\left(\phi^{4}\right), \\
\sinh (\phi)_{i j k l} & =\phi_{i j k l}+\frac{1}{6} \phi_{m n[i j} \phi^{m n p q} \phi_{k l] p q}+\mathcal{O}\left(\phi^{5}\right) .
\end{aligned}
$$

The $\mathrm{SU} \backslash E_{7(7)}$ vielbein is then given by

$$
V_{i j k l}=\operatorname{Esh}_{\phi}(d \phi)_{i j k l},
$$

where $\operatorname{Esh}_{\phi}$ is the linear operator

$$
\operatorname{Esh}_{\phi}(X)_{i j k l} \equiv \frac{\sinh \sqrt{\mathrm{A}_{\phi}}}{\sqrt{\mathrm{A}_{\phi}}}(X)_{i j k l}=X_{i j k l}+\frac{1}{6} \mathrm{~A}_{\phi}(X)_{i j k l}+\frac{1}{120} \mathrm{~A}_{\phi}^{2}(X)_{i j k l}+\mathcal{O}\left(\phi^{6}\right) .
$$

Here

$$
\mathrm{A}_{\phi}(X)_{i j k l} \equiv 2 \phi_{m n[i j} \phi^{m n p q} X_{k l] p q}-2 \phi_{m n[i j} \phi_{k l] p q} X^{m n p q} .
$$

is defined such that

$$
\left[\operatorname{ad}\left(\begin{array}{cc}
0 & \phi_{m n p q} \\
\phi^{m n p q} & 0
\end{array}\right)\right]^{2}\left(\begin{array}{cc}
0 & X_{i j k l} \\
X^{i j k l} & 0
\end{array}\right)=\left(\begin{array}{cc}
0 & \mathrm{~A}_{\phi}(X)_{i j k l} \\
\mathrm{~A}_{\phi}(X)^{i j k l} & 0
\end{array}\right),
$$

The central charge of $\mathcal{N}=8$ supergravity is a complex antisymmetric tensor $Z_{i j}$. It can always be rotated via a suitable $\mathrm{SU}(8)$ transformation into the form

$$
R_{i}^{k} R_{j}^{l} Z_{k l} \hat{=} \frac{1}{2} e^{i \varphi}\left(\begin{array}{cc}
0 & 1 \\
-1 & 0
\end{array}\right) \otimes\left(\begin{array}{cccc}
\rho_{0} & 0 & 0 & 0 \\
0 & \rho_{1} & 0 & 0 \\
0 & 0 & \rho_{2} & 0 \\
0 & 0 & 0 & \rho_{3}
\end{array}\right)
$$

such that $\rho_{0} \geq \rho_{1} \geq \rho_{2} \geq \rho_{3}$ are positive real numbers. $\varphi$ is defined (modulo $\frac{\pi}{2}$ ) as the $\mathrm{SU}(8)$ invariant function

$$
\varphi=\frac{1}{4} \arg [\operatorname{Pfaff}(Z)], \quad \operatorname{Pfaff}(Z) \equiv \frac{1}{16 \cdot 4 !} \varepsilon^{i j k l m n p q} Z_{i j} Z_{k l} Z_{m n} Z_{p q},
$$


and the four $\mathrm{SU}(8)$ invariant functions $\rho_{0}{ }^{2}, \rho_{1}{ }^{2}, \rho_{2}{ }^{2}, \rho_{3}{ }^{2}$ are the four roots of the polynomial

$$
\begin{aligned}
\lambda^{4} & -2 Z_{i j} Z^{i j} \lambda^{3}+\left(2\left(Z_{i j} Z^{i j}\right)^{2}-4 Z_{i j} Z^{j k} Z_{k l} Z^{l i}\right) \lambda^{2} \\
& -\left(\frac{4}{3}\left(Z_{i j} Z^{i j}\right)^{3}-8 Z_{i j} Z^{i j} Z_{k l} Z^{l p} Z_{p q} Z^{q k}-\frac{32}{3} Z_{i j} Z^{j k} Z_{k l} Z^{l p} Z_{p q} Z^{q i}\right) \lambda+16|\operatorname{Pfaff}(Z)|^{2} \\
& =\left(\lambda-\rho_{0}{ }^{2}\right)\left(\lambda-\rho_{1}^{2}\right)\left(\lambda-\rho_{2}{ }^{2}\right)\left(\lambda-\rho_{3}{ }^{2}\right)
\end{aligned}
$$

The $E_{7(7)}$ quartic invariant [42]

$$
\diamond(Z)=16\left(Z_{i j} Z^{j k} Z_{k l} Z^{l i}-\frac{1}{4}\left(Z_{i j} Z^{i j}\right)^{2}+4(\operatorname{Pfaff}(Z)+\overline{\operatorname{Pfaff}(Z)})\right)
$$

can be expressed in terms of the $\mathrm{SU}(8)$ invariants as

$$
\diamond(Z)=\sum_{n=0}^{3} \rho_{n}{ }^{4}-2 \sum_{m>n} \rho_{m}{ }^{2} \rho_{n}{ }^{2}+8 \rho_{0} \rho_{1} \rho_{2} \rho_{3} \cos (4 \varphi) .
$$

Being $E_{7(7)}$ invariant, it is a function of the electromagnetic charges alone and independent of the moduli. In (2.68) below, we shall define a different parametrization of the central charge $Z_{i j}$, which plays the same role for non-BPS black holes as (2.11) for BPS ones.

\subsection{Spherically symmetric, weakly extremal solutions}

As explained in [27], the dimensional reduction of $\mathcal{N}=8$ supergravity along the time direction leads to a non-linear sigma model on

$$
\mathcal{M}_{3}^{*} \cong \operatorname{Spin}_{\mathrm{c}}^{*}(16) \backslash E_{8(8)},
$$

where $\operatorname{Spin}_{\mathrm{c}}^{*}(16)$ is the quotient of $\operatorname{Spin}^{*}(16)$ by the $\mathbb{Z}_{2}$ subgroup that acts trivially in the chiral Weyl representation. To parametrize this space in a way suited to the dimensional reduction, recall that the Lie algebra $\mathfrak{e}_{8(8)}$ admits the real five-graded decomposition

$$
\mathfrak{e}_{8(8)} \cong \mathbf{1}^{(-2)} \oplus \mathbf{5 6}^{(-1)} \oplus\left(\mathfrak{g l}_{1} \oplus \mathfrak{e}_{7(7)}\right)^{(0)} \oplus \mathbf{5 6}^{(1)} \oplus \mathbf{1}^{(2)}
$$

such that $\mathfrak{e}_{7(7)}$ is the Lie algebra of the four-dimensional duality group, and $\mathfrak{s l}_{2} \cong \mathbf{1}^{(-2)} \oplus$ $\mathfrak{g l}_{1}{ }^{(0)} \oplus \mathbf{1}^{(2)}$ the Lie algebra of the Ehlers duality group for stationary solutions. We write the generators of $\mathfrak{e}_{7(7)} \cong \mathfrak{s u}(8) \oplus \mathbf{7 0}$ as $\mathbf{G}_{I}{ }^{J}, \mathbf{G}_{I J K L}$ and the ones of $\mathfrak{s l}_{2} \cong \mathbf{1}^{(-2)} \oplus \mathfrak{g l}_{1}^{\left({ }^{(0)}\right.} \oplus \mathbf{1}^{(2)}$ as $\mathbf{F}, \mathbf{H}, \mathbf{E}$, respectively. The generators of grade 1 and -1 will be written as $\mathbf{E}_{I J}, \mathbf{E}^{I J}$ and $\mathbf{F}_{I J}, \mathbf{F}^{I J}$, such that they only appear in $\mathfrak{e}_{8(8)}$ through the combinations

$$
X_{I J} \mathbf{E}^{I J}-X^{I J} \mathbf{E}_{I J}, \quad Y_{I J} \mathbf{F}^{I J}-Y^{I J} \mathbf{F}_{I J}
$$

The negative weight part of the the five-graded decomposition (2.17)

$$
\mathfrak{p} \cong \mathbf{1}^{(-2)} \oplus \mathbf{5} \mathbf{6}^{(-1)} \oplus\left(\mathfrak{g l}_{1} \oplus \mathfrak{e}_{7(7)}\right)^{(0)}
$$

defines the Lie algebra of a maximal parabolic subgroup $\mathfrak{P} \subset E_{8(8)}$, also known as the Heisenberg parabolic. $\mathrm{SU} \backslash \mathfrak{P}$ is isomorphic to the Riemannian symmetric space $\mathcal{M}_{3} \cong$ 
$\operatorname{Spin}_{\mathrm{c}}(16) \backslash E_{8(8)}$ by the Iwasawa decomposition, and to a dense subset of the pseudoRiemannian symmetric space $\mathcal{M}_{3}^{*}$. A generic element of $\mathrm{SU} \backslash \mathfrak{P}$ may be parametrized as

$$
\mathcal{V}=\operatorname{Ad}(v) \exp (U \mathbf{H}) \exp \left(\sigma \mathbf{F}+\Phi_{I J} \mathbf{F}^{I J}-\Phi^{I J} \mathbf{F}_{I J}\right)
$$

where $U$ is identified as the scale factor in the metric ansatz (1.2), $v$ is the coset representative in (2.1), $\sigma$ the NUT scalar, and $\Phi^{I J}$ are linear combinations of the Wilson lines $\zeta, \tilde{\zeta}$ transforming as an antisymmetric complex tensor of $\mathrm{SU} \subset E_{7(7)}$. The associated Maurer-Cartan form decomposes into its coset and $\mathfrak{s o}^{*}(16)$ components according to

$$
-\dot{\mathcal{V}} \mathcal{V}^{-1}=B+P \quad, \quad B \in \mathfrak{s o}^{*}(16), \quad P \in \mathfrak{e}_{8(8)} \ominus \mathfrak{s o}^{*}(16)
$$

A straightforward computation gives

$$
\begin{aligned}
-P= & \dot{U} \mathbf{H}+\frac{1}{2} e^{-2 U}\left(\dot{\sigma}+\frac{i}{2}\left(\Phi^{I J} \dot{\Phi}_{I J}-\Phi_{I J} \dot{\Phi}^{I J}\right)\right)(\mathbf{F}+\mathbf{E}) \\
& +\frac{1}{2} e^{-U}\left(\left(u_{i j}{ }^{I J} \dot{\Phi}_{I J}-v_{i j I J} \dot{\Phi}^{I J}\right)\left(\mathbf{F}^{i j}-\mathbf{E}^{i j}\right)-\left(u_{I J}^{i j} \dot{\Phi}^{I J}-v^{i j I J} \dot{\Phi}_{I J}\right)\left(\mathbf{F}_{i j}-\mathbf{E}_{i j}\right)\right) \\
& +\frac{1}{24}\left(u_{i j}{ }^{I J} \dot{v}_{k l I J}-v_{i j I J} \dot{u}_{k l}^{I J}\right) \mathbf{G}^{i j k l}
\end{aligned}
$$

where the $\mathfrak{e}_{8(8)}$ generators with lowercase indices $i, j, \cdots$ satisfy the same commutations rules as the ones with capital indices, and are related to the latter via a fixed vielbein $v$, chosen to be the vielbein $v_{0}$ at spatial infinity.

The equations of motion for weakly extremal spherically symmetric solutions (including those with non-zero NUT charge) then take the manifestly $E_{8(8)}$ invariant form

$$
\operatorname{Tr} P^{2}=0, \quad \frac{\partial}{\partial \tau}\left(\mathcal{V}^{-1} P \mathcal{V}\right)=0
$$

Defining the $\mathfrak{e}_{8(8)}$-valued Noether charge as

$$
Q \equiv \mathcal{V}^{-1} P \mathcal{V}
$$

we can characterize spherically symmetric weakly extremal black holes by the constant value of $Q$, subject to $\operatorname{Tr} Q^{2}=0$, and the asymptotic value of the scalars fields $v_{0} \in E_{7(7)}$ at spatial infinity (i.e. at $\tau=0$ ). The condition of smoothness of the metric puts additional restrictions on the Noether charge discussed in the next subsection. For our purposes it will be more convenient to characterize the solutions instead in terms of the value $P_{0}$ of $P$ at $\tau=0$.

$P_{0}$ transforms as a Majorana-Weyl spinor under $\operatorname{Spin}_{\mathrm{c}}^{*}(16)$. It can be conveniently parametrized using a fermionic oscillator basis [24],

$$
\begin{aligned}
\left|P_{0}\right\rangle & =\left(W+Z_{i j} a^{i} a^{j}+\Sigma_{i j k l} a^{i} a^{j} a^{k} a^{l}+\frac{1}{6 !} \varepsilon_{i j k l m n p q} Z^{p q} a^{i} \cdots a^{n}+\frac{1}{8 !} \varepsilon_{i j k l m n p q} \bar{W} a^{i} \cdots a^{q}\right)|0\rangle \\
& =(1+\star)\left(W+Z_{i j} a^{i} a^{j}+\frac{1}{2} \Sigma_{i j k l} a^{i} a^{j} a^{k} a^{l}\right)|0\rangle
\end{aligned}
$$

where $\star$ is the anti-involution defining the chiral Majorana-Weyl representation of $\operatorname{Spin}^{*}(16), W=M+i k$ where $M$ is the mass and $k$ the NUT charge, $Z_{i j}$ are the supersymmetric central charges and $\Sigma_{i j k l}$ are the "scalar charges". 
We will focus on the case of static solutions, such that $k=0$ and the equations of the electromagnetic fields reduce to ${ }^{12}$

$$
\frac{\partial}{\partial \tau} e^{-2 U}\left(\dot{\Phi}_{I J}-2 v_{i j I J}\left(u_{K L}^{i j} \dot{\Phi}^{K L}-v^{i j K L} \dot{\Phi}_{K L}\right)\right)=0
$$

These integrals of motion define the complex electromagnetic charges $Q_{I J} \in \mathbf{2 8}_{\mathbb{C}}$. This allows to replace the 'electromagnetic component' of $P$ by the central charges $Z(v)_{i j}$, as follows

$$
-e^{-U}\left(u_{i j}{ }^{I J} \dot{\Phi}_{I J}-v_{i j I J} \dot{\Phi}^{I J}\right)=e^{U}\left(u_{i j}{ }^{I J} Q_{I J}+v_{i j I J} Q^{I J}\right)=e^{U} Z(v)_{i j}
$$

Within the fermionic oscillator basis, $P$ then reduces to the following Majorana-Weyl spinor

$$
|P\rangle=(1+\star)\left(-\dot{U}+e^{U} Z(v)_{i j} a^{i} a^{j}-\frac{1}{24}\left(u_{i j}^{I J} \dot{v}_{k l I J}-v_{i j I J} \dot{u}_{k l}{ }^{I J}\right) a^{i} a^{j} a^{k} a^{l}\right)|0\rangle
$$

Smoothness of the metric requires $Q$, and therefore $P=\mathcal{V} Q \mathcal{V}^{-1}$, to be nilpotent of degree five when evaluated in the $\mathbf{3 8 7 5}$ representation of $\mathfrak{e}_{8(8)}$ [24],

$$
\left[P_{\mid \mathbf{3 8 7 5}}\right]^{5}=0 \text {. }
$$

This condition is invariant under the adjoint action $P \rightarrow g^{-1} P g$ where $g \in E_{8(8)}$, and therefore defines an adjoint orbit of $E_{8(8)}$. Moreover, the $\operatorname{Spin}_{\mathrm{c}}^{*}(16) \subset E_{8(8)}$ orbit of $P$ defines a Lagrangian subspace of the adjoint orbit, for the Kirillov-Kostant symplectic form induced by the Killing norm [24]. The adjoint orbits of elements of $\mathfrak{e}_{8(8)}$ satisfying the nilpotency conditions (2.29) are in one-to-one correspondence with the $\operatorname{Spin}_{\mathrm{c}}^{*}(16)$ orbits of spherically symmetric black holes satisfying $v_{0}=\mathbb{1}$. Other solutions with general value of $v_{0}$ can be obtained by acting further with $E_{7(7)}$. The stratification of the moduli space of extremal black holes solutions with $v_{0}=\mathbb{1}$ is identical to that of the corresponding $E_{8(8)}$ nilpotent orbits [24, 44], and is displayed in figure 1.

There are two $E_{8(8)}$ orbits associated to the nilpotency condition (2.29), whose union is dense in the space of solutions of this equation. They both lie in a single $E_{8}(\mathbb{C})$ orbit, labelled by the weighted Dynkin diagram $\left[\begin{array}{l}0.80002 \\ \text {, }\end{array}{ }^{13}\right.$ associated to the same five graded decomposition as in (2.17), up to a rescaling of the grading generator by two,

$$
\mathfrak{e}_{8(8)} \cong \mathbf{1}^{(-4)} \oplus \mathbf{5 6}^{(-2)} \oplus\left(\mathfrak{g l}_{1} \oplus \mathfrak{e}_{7(7)}\right)^{(0)} \oplus \mathbf{5 6}^{(2)} \oplus \mathbf{1}^{(4)}
$$

A representative $\mathbf{E}$ of the nilpotent orbit $[0000002]$ is a generic element of the grade two component $\mathbf{5 6}^{(2)}$. There are two classes of such elements which are distinguished by their isotropy subgroup inside $E_{7(7)}$, respectively $E_{6(2)}$ and $E_{6(6)}[38,41] .{ }^{14}$ Each of these two

\footnotetext{
${ }^{12}$ Where we used the identities $u^{i j}{ }_{I J} u_{i j}{ }^{K L}-v_{i j I J} v^{i j K L}=\delta_{I J}^{K L}$ and $u^{i j}{ }_{I J} v_{i j K L}=v_{i j I J} u_{K L}^{i j}$ [43].

${ }^{13}$ In general, a complex nilpotent orbit of $G_{\mathbb{C}}$ is uniquely labelled by a weighted Dynkin diagram of $G$, which records the coordinates of the Cartan generator of the $\operatorname{SL}(2, \mathbb{C})$ triplet defining the orbit. Real orbits are generally uniquely labelled by a pair of weighted Dynkin diagrams for $G$ and its maximal compact subgroup. See e.g. [40] for a thorough introduction to nilpotent orbits.

${ }^{14}$ Note however that in the case of physical interest, the graded decomposition (2.30) is defined with respect to a $\mathfrak{g l}_{1}$ subalgebra of $\mathfrak{s o}^{*}(16)$ such that the corresponding component $\mathfrak{e}_{7(7)}^{(0)}$ is not the four-dimensional duality group. Nevertheless, one checks that the isotropy subgroup of the corresponding electromagnetic charges $Z_{i j}$ defining $P$ are also left invariant by the same subgroups of $E_{7(7)}$.
} 


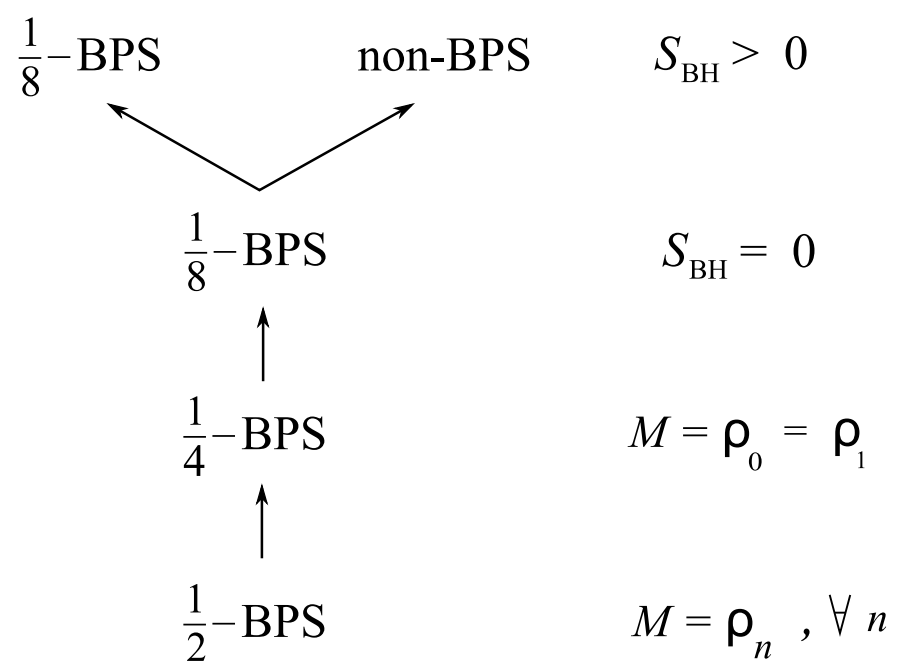

Figure 1. Stratification of the moduli space of extremal solutions in $\mathcal{N}=8$ supergravity.

$E_{8(8)}$ orbits does not contain a unique $\operatorname{Spin}_{\mathrm{c}}^{*}(16)$ orbit. Nonetheless, there is one single $\operatorname{Spin}_{\mathrm{c}}^{*}(16)$ orbit of regular spherically symmetric black holes in each $E_{8(8)}$ orbit [24]. As for the $E_{8(8)}$ orbits, we can label them by the associated $\mathfrak{s o}^{*}(16)$ weighted Dynkin diagrams. An $\mathfrak{s o}^{*}(16)$ weighted Dynkin diagram defines the coordinates of a $\mathfrak{g l}_{1}$ generator $\mathbf{h}$ of a chosen Cartan subalgebra of $\mathfrak{s o}^{*}(16)$ which defines a corresponding graded decomposition of $\mathfrak{s o}^{*}(16)$ and its Majorana-Weyl representation $\mathbf{1 2 8}_{+}$such that a representative of the orbit lies in the component of grade two of $\mathbf{1 2 8}_{+}$, in particular

$$
\mathbf{h}\left|P_{0}\right\rangle=2\left|P_{0}\right\rangle \text {. }
$$

The two orbits of $\operatorname{Spin}_{\mathrm{c}}^{*}(16)$ associated to generic extremal black holes (i.e. black holes with a non-vanishing horizon area) are labelled by $[.0800020]$ and $[.2000000]$, respectively. The aim of this section is to solve explicitly eq. (2.31) at all values of $\tau$, re-express it as a system of first order differential equations for $U$ and $v$, and read off the fake superpotential for both BPS and non-BPS extremal black holes.

\section{$2.3 \quad 1 / 8-B P S$ black holes}

For a generic 1/8-BPS spherically symmetric black hole, $P_{0}$ lies in the $\operatorname{Spin}^{*}(16)$ orbit [24]

$$
\frac{\operatorname{Spin}^{*}(16)}{(\mathrm{SU}(2) \times \mathrm{SU}(6)) \ltimes\left((\mathbf{2} \otimes \mathbf{6})^{(2)} \oplus \mathbb{R}^{(4)}\right)} \subset \frac{E_{8(8)}}{E_{6(2)} \ltimes\left((\mathbb{R} \oplus \mathbf{2 7})^{(2)} \oplus \mathbb{R}^{(4)}\right)}
$$

labelled by the weighted Dynkin diagram $[.0800020]$, associated to the five graded decomposition of $\mathfrak{s o}^{*}(16)$

$$
\mathfrak{s o}^{*}(16) \cong \mathbf{1}^{(-4)} \oplus(\mathbf{2} \otimes \mathbf{1 2})_{\mathbb{R}}^{(-2)} \oplus\left(\mathfrak{g l}_{1} \oplus \mathfrak{s u}(2) \oplus \mathfrak{s o}^{*}(12)\right)^{(0)} \oplus(\mathbf{2} \otimes \mathbf{1 2})_{\mathbb{R}}^{(2)} \oplus \mathbf{1}^{(4)}
$$

In the symmetric gauge (in which $\mathcal{V}=\exp \left(-P_{0} \tau\right)$ ), $P=P_{0}$. Since $P$ transforms as a Majorana-Weyl spinor with respect to $\operatorname{Spin}^{*}(16)$, it follows that $P$ lies in the same $\operatorname{Spin}^{*}(16)$ orbit for any value of $\tau$ in the parabolic gauge. 
The generator $\mathbf{h}_{\frac{1}{8}}$ defining the grading (2.33) through $\mathfrak{g l}_{1} \equiv \mathbb{R} \mathbf{h}_{\frac{1}{8}}$ associated to $1 / 8$ BPS solutions can be identified as follows. Let $\omega_{i j}$ be an antisymmetric tensor of rank two of SU(8) such that $I_{i}^{j} \equiv \omega_{i k} \omega^{j k}$ is a projector onto the two-dimensional subspace $\mathbb{C}^{2} \subset \mathbb{C}^{8}$ of the preserved Killing spinors at infinity (i.e. at $\tau \rightarrow 0$ ),

$$
\epsilon_{\alpha}^{i}+\varepsilon_{\alpha \beta} \omega^{i j} \epsilon_{j}^{\beta}=0 \quad \Rightarrow \quad \epsilon_{\alpha}^{i}=I_{j}^{i} \epsilon_{\alpha}^{j} .
$$

Here we have written the Killing spinor as a complex $\mathrm{SU}(2)$ spinor valued in the fundamental of $\mathrm{SU}(8)$. It transforms as a real $(\mathbf{2}, \mathbf{1 6})$ under $\mathrm{SU}(2) \times S O^{*}(16)$, where the real structure is given by the product of the pseudo-real structures on both factors. It can be then checked that the generator $\mathbf{h}_{\frac{1}{8}}$,

$$
\mathbf{h}_{\frac{1}{8}} \equiv \omega_{i j} a^{i} a^{j}-\omega^{i j} a_{i} a_{j}
$$

generates the 5-grading (2.33).

Solutions to (2.31) are $\operatorname{Spin}^{*}(12)$ Majorana-Weyl spinors of the form

$$
\left|P_{0}\right\rangle=(1+\star)\left(1+\frac{1}{2} \omega_{i j} a^{i} a^{j}\right)\left(W+Z_{i j}^{\prime} a^{i} a^{j}\right)|0\rangle
$$

where $W=M+i k$ and $Z_{i j}^{\prime}$ is the component of the central charge $Z_{i j}$ at $\tau=0$ orthogonal to $\omega_{i j}$ (i.e. $I_{i}^{k} Z_{j k}^{\prime}=0$ ). The data $\omega_{i j} \subset(\mathrm{SU}(2) \times \mathrm{SU}(6)) \backslash \mathrm{SU}(8), W \in \mathbb{C}$ and $Z_{i j}^{\prime} \in \bigwedge^{2} \mathbb{C}^{6}$ provide a complete parametrization of the $\operatorname{Spin}^{*}(16)$ orbit (2.32). To see this, note that the only generators of $\mathfrak{s o}^{*}(16)$ that act non-trivially on a representative of the grade two component $\mathbf{3 2}^{(2)}$ associated to a $\mathfrak{g l}_{1}$ generator (2.35) are in the subalgebra of negative grade

$$
\mathbf{1}^{(-4)} \oplus(\mathbf{2} \otimes \mathbf{1 2})_{\mathbb{R}}^{(-2)} \oplus\left(\mathfrak{g l}_{1} \oplus \mathfrak{s o}^{*}(12)\right)^{(0)} \subset \mathfrak{s o}^{*}(16) .
$$

By construction, the component of grade zero leaves invariant $\mathbf{h}_{\frac{1}{8}}$ and acts transitively on the components $W, Z_{i j}^{\prime}$ associated to regular black holes. The generators of strictly negative grade can be redefined through the addition of generators of strictly positive grade such that they correspond precisely to the generators of $\mathfrak{s u}(8)$ that act non-trivially on $\omega_{i j}(\mathfrak{s u}(8) \cong \mathfrak{s u}(2) \oplus \mathfrak{s u}(6) \oplus \mathbf{1} \oplus(\mathbf{2} \otimes \mathbf{6}))$.

Comparing the general form of $|P\rangle(2.28)$ to the general representative of the relevant nilpotent orbit (2.36), we conclude that in the static case (i.e. $k=0$ ), the field dependent $\mathbf{h}(\phi)$ generator can be defined at any value of $\tau$ uniquely from the the central charge. Using the decomposition (2.11) of the central charge $Z_{i j}$,

$$
\begin{aligned}
Z_{i j} & =\frac{1}{2} e^{i \varphi(\phi)} R_{i}{ }^{k}(\phi) R_{j}{ }^{l}(\phi)\left(\rho_{0}(\phi) \omega_{i j}^{o}+\rho_{1}(\phi) \omega_{i j}^{1}+\rho_{2}(\phi) \omega_{i j}^{2}+\rho_{3}(\phi) \omega_{i j}^{3}\right) \\
& =\frac{1}{2} e^{i \varphi(\phi)} R_{i}{ }^{k}(\phi) R_{j}{ }^{l}(\phi) \rho_{0}(\phi) \omega_{i j}^{0}+Z_{i j}^{\prime}
\end{aligned}
$$

where $\rho_{0}(\phi) \geq \rho_{n}(\phi)$, the semi-simple generator $\mathbf{h}(\phi)$ can be written

$$
\mathbf{h}_{\frac{1}{8}}(\phi) \equiv \omega_{i j}(\phi) a^{i} a^{j}-\omega^{i j}(\phi) a_{i} a_{j}, \quad \omega_{i j}(\phi) \equiv e^{i \varphi(\phi)} R_{i}{ }^{k}(\phi) R_{j}{ }^{l}(\phi) \omega_{i j}^{0},
$$

while

$$
|P\rangle=(1+\star)\left(1+\frac{1}{2} \omega_{i j}(\phi) a^{i} a^{j}\right) e^{U}\left(\rho_{0}(\phi)+Z_{i j}^{\prime}(\phi) a^{i} a^{j}\right)|0\rangle
$$


In the remainder, we shall refrain from writing the dependence on $\phi$ explicitely.

Comparing (2.40) and (2.28), we see that the scalar fields $U$ and $v$ satisfy the first order equations

$$
\begin{aligned}
\dot{U} & =-e^{U} \rho_{0} \\
u_{i j}{ }^{I J} \dot{v}_{k l I J}-v_{i j I J} \dot{u}_{k l}^{I J} & =-6 e^{U}\left(\omega_{[i j} Z_{k l]}+\frac{1}{24} \varepsilon_{i j k l m n p q} \omega^{m n} Z^{p q}\right)
\end{aligned}
$$

The first equation in (2.41) identifies the fake superpotential for BPS black holes as

$$
W=\rho_{0} .
$$

In order to verify that (2.41) is indeed the gradient flow of $W$, one may use the second equation in (2.39) to write $W=\omega^{i j} Z_{i j}$. One then computes (in symmetric gauge, but the proof is easily generalized to any other parametrisation of $\mathcal{M}_{4}$ )

$$
\begin{aligned}
d W= & \omega^{i j}\left(d u_{i j}{ }^{I J} Q_{I J}+d v_{i j I J} Q^{I J}\right)+\omega^{0 k l} d\left(e^{-i \varphi} R_{k}^{i} R^{j}{ }_{l}\right) Z_{i j} \\
= & \omega^{i j}\left(u_{i j}{ }^{I J} d v_{k l I J}-v_{i j I J} d u_{k l}{ }^{I J}\right) Z^{k l}+\omega^{i j}\left(u^{k l}{ }_{I J} d u_{i j}{ }^{I J}-v^{k l I J} d v_{i j I J}\right) Z_{k l} \\
& -i \omega^{i j} Z_{i j} d \varphi-2 \omega^{i k} R^{j}{ }_{l} d R_{i}{ }^{l} Z_{j k} \\
= & \operatorname{Esh}_{\phi}(d \phi)_{i j k l} \omega^{i j} Z^{k l}-i W d \varphi \\
& +\omega^{i k}\left[\frac{2}{3}\left(u^{j l}{ }_{I J} d u_{i l}{ }^{I J}-v^{j l I J} d v_{i l I J}\right)-2 R^{j}{ }_{l} d R_{i}{ }^{l}\right] Z_{j k},
\end{aligned}
$$

where we used the fact that the right-hand-side in the second line is an element of $\mathfrak{s u}(8)$. $\omega_{i j}$ is invariant under an $\mathfrak{s u}(2) \oplus \mathfrak{s u}(6) \subset \mathfrak{s u}(8)$. Moreover, the action of any generator of $\mathfrak{s u}(8)$ on $\omega_{i j}$ gives an antisymmetric tensor with at least one index in the image of the projector $I_{i}^{j} \equiv \omega_{i k} \omega^{j k}$. Using the explicit form of $Z_{i j}$ (2.38), it then follows that the very last line of (2.43) can be rewritten as

$$
\begin{aligned}
\omega^{i k}\left[\frac{2}{3}\left(u^{j l}{ }_{I J} d u_{i l}{ }^{I J}-v^{j l I J} d v_{i l I J}\right)\right. & \left.-2 R^{j}{ }_{l} d R_{i}{ }^{l}\right] \\
& =Z_{j k}^{i}\left[\frac{1}{3}\left(u^{j k}{ }_{I J} d u_{i k}{ }^{I J}-v^{j k I J} d v_{i k I J}\right)-R^{j}{ }_{k} d R_{i}{ }^{k}\right] W
\end{aligned}
$$

Besides, using the fact that $W$ is real, we compute in the same way that

$$
\begin{aligned}
d W= & \omega_{i j}\left(d u^{i j}{ }_{I J} Q^{I J}+d v^{i j I J} Q_{I J}\right)+\omega_{k l} d\left(e^{i \varphi} R_{i}{ }^{k} R_{j}{ }^{l}\right) Z_{i j} \\
= & \operatorname{Esh}_{\phi}(d \phi)^{i j k l} \omega_{i j} Z_{k l}+i W d \varphi \\
& +I_{i}^{j}\left[\frac{1}{3}\left(u_{j k}{ }^{I J} d u^{i k}{ }_{I J}-v_{j k I J} d v^{i k I J}\right)-R_{j}{ }^{k} d R_{k}^{i}\right] W
\end{aligned}
$$

Adding the two expressions of $d W$ and canceling terms, we finally obtain

$$
d W=\frac{1}{2} \operatorname{Esh}_{\phi}(d \phi)_{i j k l}\left(\omega^{i j} Z^{k l}+\frac{1}{24} \varepsilon^{i j k l m n p q} \omega_{m n} Z_{p q}\right) .
$$


It follows that the equations (2.41) can be expressed as

$$
\dot{U}=-e^{U} W, \quad \dot{\phi}_{i j k l}=-12 e^{U} \operatorname{Esh}_{\phi}^{-2}\left(\frac{\partial W}{\partial \phi}\right){ }_{i j k l}
$$

as expected. Thus, the radial evolution of the radius $U$ and scalars $v$ for $1 / 8$-BPS solutions follows the gradient flow of $W$ given in (2.42). We have therefore reproduced the result of [13] using the technology of nilpotent orbits.

Substituting (2.47) back into (2.28), one can rewrite the momentum vector as

$$
|P\rangle=e^{U}(1+\star)\left(W+Z_{i j} a^{i} a^{j}+\frac{1}{2} \operatorname{Esh}_{\phi}^{-1}\left(\frac{\partial W}{\partial \phi}\right){ }_{i j k l} a^{i} a^{j} a^{k} a^{l}\right)|0\rangle
$$

By virtue of the attractor mechanism, the scalar fields at the horizon lie at an extremum of $W$. Thus, $P$ reduces to

$$
|P\rangle \sim e^{U}(1+\star)\left(W_{*}+Z_{i j *} a^{i} a^{j}\right)|0\rangle
$$

as $\tau$ goes to infinity. From (2.36) we conclude that $Z_{i j *}^{\prime}=0$ at the horizon, and therefore

$$
\rho_{1}\left(Z_{i j *}\right)=\rho_{2}\left(Z_{i j *}\right)=\rho_{3}\left(Z_{i j *}\right)=0 .
$$

Since the $E_{7(7)}$ invariant $\diamond(Z)$ depends only on the electromagnetic charges, it is constant along the flow, and equals to $\rho_{0}\left(Z_{i j *}\right)^{4}$ at the horizon. Therefore, the value of $W$ at the horizon can be rewritten as

$$
W_{*}=\sqrt[4]{\diamond\left(Q_{I J}\right)}
$$

Using (1.7), one recovers the famous formula for the entropy of BPS black holes in $\mathcal{N}=8$ supergravity [45].

Finally, we note that the two-form $\omega_{i j}$ associated to the central charge is invariant under $\mathrm{SU}(2) \times \mathrm{SU}(6) \subset \mathrm{SU}(8)$ and $E_{6(2)} \subset E_{7(7)}$, respectively. Therefore the "fake superpotential" $W$ admits flat directions homeomorphic to the symmetric space

$$
\mathcal{M}_{\mathrm{BPS}} \cong(\mathrm{SU}(2) \times \mathrm{SU}(6)) \backslash E_{6(2)} \subset \mathcal{M}_{4}
$$

which are not determined by the attractor mechanism, but depend on the asymptotic value of the scalars $[39,46]$. In order to see this in our formalism, note from $(2.36)$ that in terms of representations of the $\mathrm{SU}(2) \times \mathrm{SU}(6)$ isotropy subgroup of $\omega_{i j}$, the components of $u_{i j}{ }^{I J} d v_{k l I J}-v_{i j I J} d u_{k l}^{I J}$ are only non-zero along the component $\mathbf{1 5}_{\mathbb{C}} \subset \mathbf{7 0}$, and are always zero along $(\mathbf{2} \otimes \mathbf{2 0})_{\mathbb{R}} \subset \mathbf{7 0}$. One therefore understands from the decomposition

$$
\begin{array}{rlrl}
\mathfrak{e}_{7(7)} & \cong \mathfrak{u}(1) \oplus \quad \mathfrak{e}_{6(2)} & \oplus \quad \mathbf{2 7} \\
& \cong \mathfrak{u}(1) \oplus \mathfrak{s u}(2) \oplus \mathfrak{s u}(6) \oplus(\mathbf{2} \otimes \mathbf{2 0})_{\mathbb{R}} \oplus \mathbf{2} \otimes \mathbf{6} \oplus \mathbf{1 5}
\end{array}
$$

that the 40 flat directions generate a $\mathcal{M}_{\mathrm{BPs}}$ subspace. 


\subsection{Non-BPS extremal black holes}

Non-supersymmetric extremal spherically symmetric black holes can be treated in much the same way. In this case, the Noether charge $Q$ (and therefore the momentum $P$ ) lies in a $\operatorname{Spin}_{\mathrm{c}}^{*}(16)$ orbit $\left[._{2000000}\right]$ of nilpotent elements of $\mathfrak{e}_{8(8)} \ominus \mathfrak{s o}^{*}(16)$, which is itself a Lagrangian submanifold of the $E_{8(8)}$ orbit [0080002] [24]

$$
\frac{\operatorname{Spin}^{*}(16)}{U S p(8) \ltimes \mathbf{2 7}} \subset \frac{E_{8(8)}}{E_{6(6)} \ltimes\left((\mathbf{1} \oplus \mathbf{2 7} \oplus \overline{\mathbf{2 7}})^{(2)} \oplus \mathbf{1}^{(4)}\right)} .
$$

The associated graded decompositions of $\mathfrak{e}_{8(8)}$ and $\mathfrak{s o}^{*}(16)$ can be read off the weighted Dynkin diagram,

$$
\begin{aligned}
& \mathfrak{s o}^{*}(16) \cong \quad \mathbf{2 8}^{(-2)} \oplus\left(\mathfrak{g l}_{1} \oplus \mathfrak{s u}^{*}(8)\right)^{(0)} \oplus \overline{\mathbf{2 8}}^{(2)} \\
& \mathfrak{e}_{8(8)} \ominus \mathfrak{s o}^{*}(16) \cong \mathbf{1}^{(-4)} \oplus \overline{\mathbf{2 8}}^{(-2)} \oplus \quad \mathbf{7 0}^{(0)} \quad \oplus \mathbf{2 8}^{(2)} \oplus \mathbf{1}^{(4)} \cong \mathbf{1 2 8}
\end{aligned}
$$

This is the same 5 -grading as the one relevant for the minimal nilpotent orbit with weighted Dynkin diagrams $([.100000],[0080001])$ associated to 1/2-BPS black holes, after rescaling the generator $\mathbf{h}$ by two (see appendix A). An orbit representative is a generic element of $\mathbf{2 8}^{(2)}$, whose stabilizer defines a $U S p(8) \subset S U^{*}(8)^{(0)}$ subgroup (equivalently, a generic element of the $\mathbf{5 6}^{(2)}$ whose stabilizer defines an $E_{6(6)} \subset E_{7(7)}^{(0)}$ subgroup). Its associated semi-simple generator takes the form

$$
\mathbf{h}_{0} \equiv \frac{1}{2}\left(e^{i \alpha} \Omega_{i j} a^{i} a^{j}-e^{-i \alpha} \Omega^{i j} a_{i} a_{j}\right),
$$

where the complex two-form $\Omega_{i j}$ satisfies ${ }^{15}$

$$
\Omega_{[i j} \Omega_{k l]}+\frac{1}{24} \varepsilon_{i j k l m n p q} \Omega^{m n} \Omega^{p q}=0, \quad \Omega_{i k} \Omega^{j k}=\delta_{i}^{j} .
$$

Indeed, using the identities

$$
\left[\mathbf{h}_{0}, a^{i} \pm e^{-i \alpha} \Omega^{i j} a_{j}\right]= \pm\left(a^{i} \pm e^{-i \alpha} \Omega^{i j} a_{j}\right)
$$

one obtains the generators of degree \pm 2 of $\mathfrak{s o}^{*}(16)$ as the 'squares' of $a^{i} \pm e^{-i \alpha} \Omega^{i j} a_{j}$, respectively, and the $\mathfrak{s u}^{*}(8)$ generators as their commutators. One can then check that the elements of $\mathbf{1}^{(4)}$ are of the form

$$
\left|\mathscr{C}_{0}^{(4)}\right\rangle=i N e^{-2 i \alpha} e^{\frac{1}{2} e^{i \alpha} \Omega_{i j} a^{i} a^{j}}|0\rangle
$$

where $N$ is real. The elements of grade two $\mathbf{h}_{0}\left|\mathscr{C}^{(2)}\right\rangle=2\left|\mathscr{C}^{(2)}\right\rangle$ can be computed to be of the form [25]

$$
\left|\mathscr{C}_{0}^{(2)}\right\rangle=(1+\star)\left(1+\frac{1}{4} e^{i \alpha} \Omega_{i j} a^{i} a^{j}\right)\left(e^{-2 i \alpha} M+e^{-i \alpha} \Xi_{i j} a^{i} a^{j}\right)|0\rangle,
$$

where $\Xi_{i j}$ satisfies

$$
\Xi_{i j}=\Omega_{i k} \Omega_{j l} \Xi^{k l}, \quad \Omega^{i j} \Xi_{i j}=0 .
$$

\footnotetext{
${ }^{15}$ Equivalently, $\rho_{0}\left(\Omega_{i j}\right)=\rho_{1}\left(\Omega_{i j}\right)=\rho_{2}\left(\Omega_{i j}\right)=\rho_{3}\left(\Omega_{i j}\right)=1$ and $\varphi\left(\Omega_{i j}\right)=\frac{\pi}{4}$.
} 
These conditions state that $\Xi_{i j}$ is an element of the real $\mathbf{2 7}$ representation of the $U S p(8)$ subgroup of $\mathrm{SU}(8)$ that leaves invariant $\Omega_{i j}$. Such elements $\left|\mathscr{C}_{0}^{(2)}\right\rangle$ correspond to special values of the central charges in the asymptotic region, whose overall phase $\varphi(Z)$ is determined by the value of the NUT charge. The most general charge with strictly negative $E_{7(7)}$ invariant $\diamond(Z)<0$ can be obtained with a linear combination of $\left|\mathscr{C}_{0}^{(2)}\right\rangle$ and $\left|\mathscr{C}_{0}^{(4)}\right\rangle$, associated to the $\mathfrak{g l}_{1}$ generator

$$
\mathbf{h} \equiv \frac{1}{2}\left(e^{i \alpha}(1-i \lambda) \Omega_{i j} a^{i} a^{j}-e^{-i \alpha}(1+i \lambda) \Omega^{i j} a_{i} a_{j}\right)+i \lambda\left(a^{i} a_{i}-4\right)
$$

In this case the general element of grade two $\left|\mathscr{C}^{(2)}\right\rangle$ reads

$$
\begin{gathered}
\left|\mathscr{C}^{(2)}\right\rangle=N(1+\star)\left(e^{-2 i \alpha}(1+i \lambda)+e^{-i \alpha} \frac{1+2 i \lambda}{4} \Omega_{i j} a^{i} a^{j}+\frac{i \lambda}{16} \Omega_{i j} \Omega_{k l} a^{i} a^{j} a^{k} a^{l}\right. \\
\left.+N^{-1}\left(1+\frac{1}{4} e^{i \alpha} \Omega_{i j} a^{i} a^{j}\right) e^{-i \alpha} \Xi_{i j} a^{i} a^{j}\right)|0\rangle
\end{gathered}
$$

with NUT charge

$$
k=N(\lambda \cos 2 \alpha-\sin 2 \alpha)
$$

The parameters $\Omega_{i j} \in U S p(8) \backslash \mathrm{SU}(8), \alpha, \lambda, N$ and $\Xi_{i j} \in \mathbf{2 7}$ give a complete parametrization of the $\operatorname{Spin}^{*}(16)$ orbit (2.54). To see this, we note that the elements of $\mathfrak{s o}^{*}(16)$ that act non-trivially on a generic element of grade two of (2.55) combine into representations of its $U S p(8) \subset S U^{*}(8)$ isotropy subgroup as

$$
(\mathbf{1} \oplus \mathbf{2 7})^{(-2)} \oplus\left(\mathfrak{g l}_{1} \oplus \mathbf{2 7}\right)^{(0)} \oplus \mathbf{1}^{(2)} \subset \mathfrak{s o}^{*}(16)
$$

The component of grade zero leaves $\mathbf{h}$ invariant by definition, and acts transitively on the set of parameters $N, \Xi_{i j}$ defining regular black holes. The generators of $\mathfrak{s u}(8)$ that act non-trivially on $\Omega_{i j}$ are given by linear combinations in $\mathbf{2 7 ^ { ( - 2 ) }} \oplus \mathbf{2 7 ^ { ( 2 ) }}$, while the remaining nilpotent generators $\mathbf{1}^{(-2)} \oplus \mathbf{1}^{(2)}$ act by shifting the two parameters $\alpha$ and $\lambda$.

Since we are interested in static solutions only, we must cancel the NUT charge (2.64) by choosing $\lambda=\tan (2 \alpha)$ (with $-\frac{\pi}{2}<\alpha<\frac{\pi}{2}$ ). The asymptotic value of $P$ thus takes the form

$$
\left|P_{0}\right\rangle=(1+\star)\left(1+\frac{1}{4} e^{i \alpha} \Omega_{i j} a^{i} a^{j}\right)\left(M\left(1+\frac{i}{4} e^{-i \alpha} \sin (2 \alpha) \Omega_{k l} a^{k} a^{l}\right)+e^{-i \alpha} \Xi_{i j} a^{i} a^{j}\right)|0\rangle
$$

where $M=\cos ^{-1}(2 \alpha) N$ is the physical mass. The asymptotic central charge $Z_{i j}$ is readily obtained from the part of (2.66) bilinear in the oscillators,

$$
Z_{i j}=\frac{1}{2}\left(e^{i \alpha}+i e^{-i \alpha} \sin 2 \alpha\right) \varrho \Omega_{i j}+e^{-i \alpha} \Xi_{i j}
$$


where $\varrho \equiv M / 2$. Due to the conditions (2.61), the complex two-form $Z_{i j}$ can brought by an $\mathrm{SU}(8)$ rotation into the skew-diagonal form

$$
\begin{aligned}
& \tilde{R}_{i}^{k} \tilde{R}_{j}^{l} Z_{k l} \hat{=} \frac{e^{\frac{i \pi}{4}}}{2}\left(\begin{array}{cc}
0 & 1 \\
-1 & 0
\end{array}\right) \otimes\left[\left(e^{i \alpha}+i e^{-i \alpha} \sin 2 \alpha\right)\left(\begin{array}{cccc}
\varrho & 0 & 0 & 0 \\
0 & \varrho & 0 & 0 \\
0 & 0 & \varrho & 0 \\
0 & 0 & 0 & \varrho
\end{array}\right)\right. \\
& \left.+e^{-i \alpha}\left(\begin{array}{cccc}
\xi_{1}+\xi_{2}+\xi_{3} & 0 & 0 & 0 \\
0 & -\xi_{1} & 0 & 0 \\
0 & 0 & -\xi_{2} & 0 \\
0 & 0 & 0 & -\xi_{3}
\end{array}\right)\right]
\end{aligned}
$$

where $\varrho>0$ and $\xi_{1}, \xi_{2}, \xi_{3} \in \mathbb{R}$ can be chosen such that

$$
\xi_{1}+\xi_{2}+\xi_{3} \geq-\xi_{1} \geq-\xi_{2} \geq-\xi_{3} .
$$

The condition of non-saturation of the BPS bound $M>\rho_{n}$ further requires that

$$
\varrho \cos 2 \alpha>\xi_{1}+\xi_{2}+\xi_{3} \geq-\xi_{1} \geq-\xi_{2} \geq-\xi_{3} .
$$

In the parametrization (2.68), the $E_{7(7)}$ quartic invariant (2.14) factorizes into

$$
\diamond(Z)=-16 \cos ^{2}(2 \alpha)\left(\varrho \cos (2 \alpha)-\xi_{1}-\xi_{2}-\xi_{3}\right) \prod_{n=1}^{3}\left(\varrho \cos (2 \alpha)+\xi_{n}\right),
$$

and is therefore strictly negative inside the tetrahedron (2.70). It vanishes whenever $M=$ $\rho_{m}$ for any $m$, i.e. when the black hole becomes BPS with vanishing horizon area. The quantities $\alpha, \varrho, \xi_{1}, \xi_{2}$ and $\xi_{3}$ define a basis of the $\mathrm{SU}(8)$ invariant functions of $Z_{i j}$ well suited for non-BPS solutions, alternative to the basis of functions $\rho_{n}, \varphi$ adapted to the BPS case. The diagonalization problem (2.68) is central to the determination of the fake superpotential for non-BPS solutions, and is reduced in appendix B to the solution of a sextic polynomial.

Comparing the general form of $|P\rangle$ (2.28) to the general representative of the relevant nilpotent orbit (2.66), we conclude that in the static case (i.e. $k=0$ ), the field dependent $\mathbf{h}$ generator is determined in terms of the central charges $Z_{i j}$. Using the decomposition (2.67),

$$
Z_{i j}=\frac{1}{2} e^{-i \alpha(\phi)} \tilde{R}_{i}^{k}(\phi) \tilde{R}_{j}^{l}(\phi)\left(\left(e^{2 i \alpha(\phi)}+i \sin (2 \alpha(\phi))\right) \varrho(\phi) \Omega_{k l}\right)+e^{-i \alpha(\phi)} \Xi_{i j}(\phi)
$$

where

$$
\Xi_{i j}(\phi)=\frac{1}{2} \tilde{R}_{i}^{k}(\phi) \tilde{R}_{j}^{l}(\phi)\left(\xi_{1}(\phi) \varpi_{k l}^{1}+\xi_{2}(\phi) \varpi_{k l}^{2}+\xi_{3}(\phi) \varpi_{k l}^{3}\right)
$$

with $\xi_{n}(\phi)$ taking values in the tetrahedron (2.70), the $\mathbf{h}$ generator is defined by

$$
\mathbf{h}=\frac{1}{2}\left(\hat{\Omega}_{i j}(\phi) a^{i} a^{j}-\hat{\Omega}^{i j}(\phi) a_{i} a_{j}\right)+i \tan (2 \alpha(\phi))\left(a^{i} a_{i}-4\right)
$$


with

$$
\hat{\Omega}_{i j}(\phi)=\frac{e^{-i \alpha(\phi)}}{\cos (2 \alpha(\phi))} \tilde{R}_{i}^{k}(\phi) \tilde{R}_{j}^{l}(\phi) \Omega_{k l}
$$

Indeed, one computes that the general solution to the equation $\mathbf{h}|P\rangle=2|P\rangle$ is

$$
|P\rangle=(1+\star)\left(1+\frac{1}{4} e^{2 i \alpha} \cos (2 \alpha) \hat{\Omega}_{i j} a^{i} a^{j}\right) e^{U}\left(2 \varrho\left(1+\frac{i}{8} \sin (4 \alpha) \hat{\Omega}_{k l} a^{k} a^{l}\right)+e^{-i \alpha} \Xi_{i j} a^{i} a^{j}\right)|0\rangle
$$

The non-BPS nilpotent orbit therefore determines the scalar fields $U$ and $v$ through the first order equations

$$
\begin{aligned}
\dot{U} & =-2 e^{U} \varrho \\
u_{i j}{ }^{I J} \dot{v}_{k l I J}-v_{i j I J} \dot{u}_{k l}^{I J} & =-6 e^{U}\left(\hat{\Omega}_{[i j} Z_{k l]}+\frac{1}{24} \varepsilon_{i j k l m n q q} \hat{\Omega}^{m n} Z^{p q}\right)
\end{aligned}
$$

The first equation identifies the fake superpotential for extremal non-BPS black holes as

$$
W=2 \varrho,
$$

where $\varrho$ is the diagonal component of the central charge $Z_{i j}$ in the non-standard diagonalization problem (2.68). In particular, $W$ is larger than the modulus of any of the eigenvalues $\rho_{m}$ of the central charge matrix, as required for a non-BPS solution. Equation (2.78) is one of the main results in this paper.

In order to check that the second line in (2.77) describes the gradient flow of $W$, we rewrite $W=\frac{1}{2} \operatorname{Re}\left[\hat{\Omega}^{i j} Z_{i j}\right]$, and compute $d W$ following the same steps as in (2.43). In this way we obtain

$$
\begin{aligned}
d W= & \frac{1}{4} \operatorname{Esh}_{\phi}(d \phi)_{i j k l}\left(\hat{\Omega}^{i j} Z^{k l}+\frac{1}{24} \varepsilon^{i j k l m n p q} \hat{\Omega}_{m n} Z_{p q}\right) \\
& +\frac{1}{2} \operatorname{Re}\left(\tilde{R}^{i}{ }_{k} \tilde{R}^{j}{ }_{l} \Omega^{k l} Z_{i j} d \frac{e^{i \alpha}}{\cos 2 \alpha}\right) \\
& +\operatorname{Re}\left(\hat{\Omega}^{i k}\left[\frac{1}{3}\left(u^{j l}{ }_{I J} d u_{i l}{ }^{I J}-v^{j l I J} d v_{i l I J}\right)-\tilde{R}^{j} d \tilde{R}_{i}{ }^{l}\right] Z_{j k}\right)
\end{aligned}
$$

One computes easily that

$$
\tilde{R}_{k}^{i} \tilde{R}_{l}^{j} \Omega^{k l} d \frac{e^{i \alpha}}{\cos 2 \alpha}=i(1-2 i \tan 2 \alpha) \hat{\Omega}^{i j} d \alpha
$$

and using the explicit form of the central charge (2.72) one computes that

$$
\frac{1}{2} \hat{\Omega}^{i j} Z_{i j}=2(1+2 i \tan (2 \alpha)) \varrho
$$

such that the second line in (2.43) cancels. The anti-hermiticity of the $\mathfrak{s u}(8)$ generators implies that

$$
\begin{aligned}
\hat{\Omega}^{i[k}\left[\frac { 1 } { 3 } \left(u_{I J}^{j] l} d u_{i l}^{I J}-\right.\right. & \left.\left.v^{j] l I J} d v_{i l I J}\right)-\tilde{R}_{l}^{j} d \tilde{R}_{i}^{l}\right] \\
& =-\stackrel{\Omega}{ }^{k p} \stackrel{\Omega}{\Omega}^{j q} \hat{\Omega}_{i[p}\left[\frac{1}{3}\left(u_{q] l}^{I J} d u^{i l}{ }_{I J}-v_{q] l I J} d v^{i l I J}\right)-\tilde{R}_{q}^{l} d \tilde{R}_{l}^{i}\right]
\end{aligned}
$$


where $\AA_{i j} \equiv \cos (2 \alpha) \hat{\Omega}_{i j}$ is normalized such that $\AA_{i k} \AA^{j k}=\delta_{i}^{j}$. With respect to $\hat{\Omega}_{i j}$, the generators of $\mathfrak{s u}^{*}(8)$ decompose into the generators of the $\mathfrak{u s p}(8)$ subalgebra that leave $\hat{\Omega}_{i j}$ invariant, and the remaining 27 generators that transform as a traceless antisymmetric tensor of rank two with respect to $U S p(8)$. It follows that

$$
\begin{aligned}
\hat{\Omega}^{i k}\left[\frac{1}{3}\left(u^{j l}{ }_{I J} d u_{i l}{ }^{I J}-v^{j l I J} d v_{i l I J}\right)-\tilde{R}_{l}{ }_{l} d \tilde{R}_{i}{ }^{l}\right] & Z_{j k} \\
& =\hat{\Omega}^{i k}\left[\frac{1}{3}\left(u^{j l}{ }_{I J} d u_{i l}{ }^{I J}-v^{j l I J} d v_{i l I J}\right)-\tilde{R}^{j}{ }_{l} d \tilde{R}_{i}^{l}\right] e^{-i \alpha} \Xi_{j k}
\end{aligned}
$$

And because

$$
e^{-i \alpha} \Xi_{i j}=\stackrel{\Omega}{\Omega}_{i k} \stackrel{\circ}{\Omega}_{j l} e^{i \alpha} \Xi^{k l}
$$

by definition (2.61), (2.83) is pure imaginary and does not contribute to (2.43). We finally obtain that

$$
d W=\frac{1}{4} \operatorname{Esh}_{\phi}(d \phi)_{i j k l}\left(\hat{\Omega}^{i j} Z^{k l}+\frac{1}{24} \varepsilon^{i j k l m n p q} \hat{\Omega}_{m n} Z_{p q}\right)
$$

such that the first order equations $(2.77)$ can be rewritten as

$$
\dot{U}=-e^{U} W, \quad \dot{\phi}_{i j k l}=-12 e^{U} \operatorname{Esh}_{\phi}{ }^{-2}\left(\frac{\partial W}{\partial \phi}\right)_{i j k l}
$$

This confirms that (2.78) is indeed a fake superpotential for the non-BPS extremal solutions.

In appendix B we discuss how $W(2.78)$ can be evaluated in practice, and show that $W^{2}$ can be obtained in general as a specific root of a polynomial (B.14) of degree six, whose coefficients are polynomial in the central charges $Z_{i j}$. At some particular loci in the $\rho_{n}, \varphi$ space however, this polynomial becomes reducible. This happens in particular when $\varphi=0$ $\bmod \pi / 4, \alpha=0$, where the sextic polynomial (B.14) becomes fully reducible. For $\varphi=\pi / 4$ $\bmod \pi / 2$, the physical root is $W_{0}$ in (B.16), i.e.

$$
\varphi=\pi / 4: \quad W=\frac{1}{2}\left(\rho_{0}+\rho_{1}+\rho_{2}+\rho_{3}\right) .
$$

This result is in agreement with [13]. Our formula (2.78) is however more general, and does not assume any restriction on the moduli nor on the charges. Similarly, at $\varphi=0$ $\bmod \pi / 2$, the physical root is the largest of $W_{n}$ in (B.29), i.e.

$$
\varphi=0: \quad W=\frac{1}{2}\left(\rho_{0}+\rho_{1}+\rho_{2}+\rho_{3}\right)-\min \left(\varrho_{m}\right) .
$$

Having obtained the fake superpotential for non-BPS black holes, one may substitute (2.86) into (2.76) and rewrite the momentum as

$$
|P\rangle=e^{U}(1+\star)\left(W+Z_{i j} a^{i} a^{j}+\frac{1}{2} \operatorname{Esh}_{\phi}{ }^{-1}\left(\frac{\partial W}{\partial \phi}\right){ }_{i j k l} a^{i} a^{j} a^{k} a^{l}\right)|0\rangle
$$

By virtue of the attractor mechanism, the scalar fields at the horizon again lie at an extremum value of $W$. It follows that

$$
|P\rangle \sim e^{U}(1+\star)\left(W_{*}+Z_{i j *} a^{i} a^{j}\right)|0\rangle
$$


as $\tau$ goes to infinity. Using (2.76), one concludes that at the horizon, the central charges satisfy

$$
\xi_{1}\left(Z_{i j *}\right)=\xi_{2}\left(Z_{i j *}\right)=\xi_{3}\left(Z_{i j *}\right)=0, \quad \alpha\left(Z_{i j *}\right)=0
$$

In terms of the $\mathrm{SU}(8)$ invariant functions appearing in the standard diagonalization (2.11), this can be expressed as

$$
\rho_{0}\left(Z_{i j *}\right)=\rho_{1}\left(Z_{i j *}\right)=\rho_{2}\left(Z_{i j *}\right)=\rho_{3}\left(Z_{i j *}\right)=\frac{1}{2} \sqrt[4]{-\diamond(Z)}, \quad \varphi\left(Z_{i j *}\right)=\frac{\pi}{4} .
$$

In particular, at the horizon

$$
W_{*}=\sqrt[4]{-\diamond\left(Q_{I J}\right)},
$$

where we again noted that $\diamond(Z)$ depends only on the the conserved charges. Using (1.7) we recover the known entropy formula for non-BPS extremal black holes in $\mathcal{N}=8$ supergravity [47].

Similarly to the BPS case, the symplectic form $\Omega_{i j}$ is invariant under $U S p(8) \subset \mathrm{SU}(8)$ and $E_{6(6)} \subset E_{7(7)}$, respectively. This implies that fake superpotential $W$ exhibits flat directions homeomorphic to the symmetric space

$$
\mathcal{M}_{\text {non-BPS }} \cong \operatorname{Sp} \backslash E_{6(6)} \text {. }
$$

along which the attractor mechanism is inactive [39, 48, 49] (here Sp is the quotient of $U S p(8)$ by its $\mathbb{Z}_{2}$ centre leaving invariant the representations of even rank). Indeed, the symplectic form $\hat{\Omega}_{i j}$ associated to the central charge is left invariant by a $U S p(8) \subset \mathrm{SU}(8)$ subgroup, with respect to which the components of $u_{i j}{ }^{I J} d v_{k l I J}-v_{i j I J} d u_{k l}{ }^{I J}$ are only nonzero along the components $\mathbb{R} \oplus \mathbf{2 7} \subset \mathbf{7 0}$, and are always zero along $\mathbf{4 2} \subset \mathbf{7 0}$ as can be seen from (2.76). From the graded decomposition

$$
\begin{aligned}
& \mathfrak{e}_{7(7)} \cong \overline{\mathbf{2 7}}^{(-2)} \oplus \quad\left(\mathfrak{g l}_{1} \oplus \mathfrak{e}_{6(6)}\right)^{(0)} \quad \oplus \mathbf{2 7}^{(2)} \\
& \cong \mathbf{2} \mathbf{7}^{(-2)} \oplus\left(\mathfrak{g l}_{1} \oplus \mathfrak{u} \mathfrak{s p}(8) \oplus \mathbf{4 2}\right)^{(0)} \oplus \mathbf{2 7}^{(2)},
\end{aligned}
$$

it is evident that the 42 flat directions at the horizon parametrize the symmetric space (2.94). It should be noticed that this moduli space of flat directions is isomorphic to the moduli space $\mathcal{M}_{5}$ of the five-dimensional supergravity model obtained after decompactification [39, 49].

We conclude this section by discussing the relation between the nilpotent orbits of the moment $P$ under $G_{3}$, and the orbits of the electromagnetic charges $Q_{I J}$ under $G_{4}$, as classified in $[38,39]$. To this aim, notice that, as an $\mathfrak{e}_{8(8)}$ element, $P$ is proportional to

$$
\mathbf{e}=\mathbf{H}+\hat{\mathbf{Z}}_{*}, \quad \hat{\mathbf{Z}}_{*} \equiv \frac{1}{2} W_{*}^{-1}\left(Z_{* i j}\left(\mathbf{F}^{i j}-\mathbf{E}^{i j}\right)-Z_{*}^{i j}\left(\mathbf{F}_{i j}-\mathbf{E}_{i j}\right)\right),
$$

at $\tau \rightarrow+\infty$. One can then use the Cartan involution $\dagger$ defining $\operatorname{Spin}_{\mathrm{c}}(16) \subset E_{8(8)}$ to complete e into a triplet $(\mathbf{e}, \mathbf{f}, \mathbf{h})$,

$$
\mathbf{f}=\mathbf{e}^{\dagger}=\mathbf{H}-\hat{\mathbf{Z}}_{*} \quad, \quad \mathbf{h}=[\mathbf{e}, \mathbf{f}]=-2\left[\mathbf{H}, \hat{\mathbf{Z}}_{*}\right] .
$$


Indeed, it follows from

$$
\left[\mathbf{H},\left[\mathbf{H}, \hat{\mathbf{Z}}_{*}\right]\right]=\hat{\mathbf{Z}}_{*} \quad, \quad\left[\hat{\mathbf{Z}}_{*},\left[\mathbf{H}, \hat{\mathbf{Z}}_{*}\right]\right]=\mathbf{H}
$$

that $[\mathbf{h}, \mathbf{e}]=2 \mathbf{e}$. The maximal reductive subgroup $J_{\mathbf{e}}^{\mathrm{R}}$ of the stabilizer of $\mathbf{e}$ in $E_{8(8)}$ is the intersection of the stabilizers of $\mathbf{e}$ and $\mathbf{h}$ in $E_{8(8)}$. The stabilizer of $\mathbf{h}$ in $E_{8(8)}$ is a $G L(1, \mathbb{R}) \times E_{7(7)}$ subgroup, such that the $E_{7(7)}$ factor is conjugate but distinct from the four-dimensional duality group $G_{4}=E_{7(7)}$. Because any element $\mathrm{x}$ of a reductive algebra $\mathfrak{j}_{\mathbf{e}}$ decomposes into two elements $\mathbf{x}_{ \pm} \in \mathfrak{j}_{\mathbf{e}}$, such that

$$
\mathbf{x}=\mathbf{x}_{+}+\mathbf{x}_{-}, \quad \mathbf{x}_{ \pm}^{\dagger}= \pm \mathbf{x}_{ \pm}
$$

$J_{\mathbf{e}}^{\mathrm{R}}$ must stabilize $\mathbf{H}$ and $\hat{\mathbf{Z}}_{*}$ separately. On the other hand, the stabilizer of $\mathbf{H}$ is $G L(1, \mathbb{R}) \times$ $G_{4}$. At the horizon (i.e. at $\left.\tau \rightarrow+\infty\right), J_{\mathbf{e}}^{\mathrm{R}}$ therefore coincides with the stabilizer of the central charge $Z_{* i j}$ inside $E_{7(7)} . J_{\mathbf{e}}^{\mathrm{R}}$ is then the intersection of the two $E_{7(7)}$ subgroups defined by $\mathbf{H}$ and $\mathbf{h}$, respectively. Away from the horizon however (at finite $\tau$ ), these two subgroups differ by a similarity transformation.

The above discussion is in fact completely general and applies for any extremal black hole with non-vanishing horizon area, in any theory admitting a symmetric scalar manifold whose isometry group acts faithfully on the electromagnetic charges. The stabilizers of the central charges computed in $[38,39]$, indeed match perfectly the reductive stabilizers of the corresponding nilpotent orbits given in [41]. We have already seen in the case of maximal supergravity that we have the following correspondence

$$
\begin{aligned}
& J_{P} \cong E_{6(2)} \ltimes\left(\mathbf{2 7}^{(2)} \oplus \mathbb{R}^{(4)}\right) \times \mathbb{R} \quad \Leftrightarrow \quad J_{Q} \cong E_{6(2)}, \\
& J_{P} \cong E_{6(6)} \ltimes\left((\mathbf{2 7} \oplus \overline{\mathbf{2 7}})^{(2)} \oplus \mathbb{R}^{(4)}\right) \times \mathbb{R} \quad \Leftrightarrow \quad J_{Q} \cong E_{6(6)},
\end{aligned}
$$

between the stabilizer $J_{P}$ of $P$ in $E_{8(8)}$ and the stabilizer $J_{Q}$ of $Q_{I J}$ in $E_{7(7)}$. In the example of the exceptional $\mathcal{N}=2$ supergravity, to be discussed in section 3.1 below, $G_{4} \cong E_{7(-25)}$ and $G_{3} \cong E_{8(-24)}$, and there are three nilpotent orbits of $E_{8(-24)}$ associated to extremal black holes, characterized by the stabilizers

$$
\begin{array}{rlll}
J_{P} \cong E_{6(-78)} \ltimes\left(\mathbf{2 7 ^ { ( 2 ) }} \oplus \mathbb{R}^{(4)}\right) \times \mathbb{R} & \Leftrightarrow & J_{Q} \cong E_{6(-78)}, \\
J_{P} \cong E_{6(-14)} \ltimes\left(\mathbf{2 7 ^ { ( 2 ) }} \oplus \mathbb{R}^{(4)}\right) \times \mathbb{R} & \Leftrightarrow \quad J_{Q} \cong E_{6(-14)}, \\
J_{P} \cong E_{6(-26)} \ltimes\left((\mathbf{2 7} \oplus \overline{\mathbf{2 7}})^{(2)} \oplus \mathbb{R}^{(4)}\right) \times \mathbb{R} & \Leftrightarrow & J_{Q} \cong E_{6(-26)} .
\end{array}
$$

\section{Truncations of maximal supergravity and their extensions}

Our method for deriving the fake superpotential of static extremal black holes applies to any symmetric model of $4 \mathrm{D}$ gravity coupled to abelian vector fields and scalar fields invariant under a continuous duality group $G_{4}$, provided $G_{4}$ acts faithfully on electromagnetic charges. Many such models can be obtained by consistent truncations of $\mathcal{N}=8$ supergravity, and all others follow by "covariantisation". In this section we shall restrict ourselves to models that can be obtained by reduction on a circle of 5D supergravity theories. Indeed, these are the only ones which admit non-BPS black holes with no central charge being 
saturated [25], for which our formalism is most fruitful. All other symmetric models have only two types of generic extremal black holes, and the fake superpotential is always of the form $W=\rho_{n}$.

\subsection{Magic $\mathcal{N}=2$ supergravity}

The maximal $\mathcal{N}=2$ truncation of $\mathcal{N}=8$ supergravity is the 'magic' supergravity theory associated to the quaternions [50]. Its bosonic sector is identical to the bosonic sector of $\mathcal{N}=6$ supergravity. Within this truncation, the central charges decompose into the $\mathcal{N}=2$ central charge $Z=2 Z_{12}$ and the $\mathcal{N}=6$ central charges $Z_{A B}$ which define an antisymmetric tensor of $\mathrm{U}(6)$, with $S(\mathrm{U}(2) \times \mathrm{U}(6)) \subset \mathrm{SU}(8)$ such that $\mathrm{U}(2)$ is the $\mathcal{N}=2$ R-symmetry group and $\mathrm{U}(6)$ the $\mathcal{N}=6$ R-symmetry group. The maximal subgroup of $E_{7(7)}$ that preserves this decomposition is $\mathrm{SU}(2) \times \operatorname{Spin}^{*}(12)_{c}$, and the scalar fields take values in

$$
\text { q) } \mathcal{M}_{4} \cong \mathrm{U}(6) \backslash \operatorname{Spin}^{*}(12)_{\mathrm{c}}, \quad \mathcal{M}_{3}^{*} \cong\left(\mathrm{SL}(2) \times \operatorname{Spin}^{*}(12)_{\mathrm{c}}\right) \backslash E_{7(-5)} \text {. }
$$

In particular, only the components (and the ones obtained by antisymmetric permutations of the indices)

$$
\phi_{12 A B} \equiv \phi_{A B}, \quad \phi_{A B C D}=\frac{1}{2} \varepsilon_{A B C D E F} \phi^{E F}
$$

are non-zero in the symmetric gauge, where $\phi_{A B}$ is an antisymmetric tensor of $\mathrm{U}(6)$.

With respect to $S(\mathrm{U}(2) \times \mathrm{U}(6)) \subset \mathrm{SU}(8)$, the representations of the fermions decompose as follows

$$
8 \cong \mathbf{2} \oplus \mathbf{6}, \quad 56 \cong \mathbf{2} \otimes \mathbf{1 5} \oplus \overline{\mathbf{6}} \oplus \mathbf{2 0},
$$

such that the fermionic fields of the $\mathcal{N}=2$ truncation are 2 gravitini and symplecticMajorana spinors in the $\mathbf{2} \otimes \mathbf{1 5}$ representation, and the fermionic fields of the $\mathcal{N}=6$ truncation are 6 gravitini and symplectic-Majorana spinors in the $\overline{\mathbf{6}} \oplus \mathbf{2 0}$.

The $\mathrm{SU}(8)$ invariant functions of the central charges $Z_{i j}, \rho_{0}, \rho_{1}, \rho_{2}, \rho_{3}$ and $\varphi$ then become $\mathrm{U}(6)$ invariant functions of $Z$ and $Z_{A B}$ defined by ${ }^{16}$

$$
\begin{aligned}
\lambda^{3}- & 2 Z_{A B} Z^{A B} \lambda^{2}+\left(2\left(Z_{A B} Z^{A B}\right)^{2}-4 Z_{A B} Z^{B C} Z_{C D} Z^{D A}\right) \lambda-\frac{1}{36}\left|\varepsilon_{A B C D E F} Z^{A B} Z^{C D} Z^{E F}\right|^{2} \\
\quad & =\left(\lambda-\rho_{1}^{2}\right)\left(\lambda-\rho_{2}^{2}\right)\left(\lambda-\rho_{3}^{2}\right)
\end{aligned}
$$

and

$$
\rho_{0}=|Z|, \quad 4 \varphi=\arg \left[Z \varepsilon^{A B C D E F} Z_{A B} Z_{C D} Z_{E F}\right] .
$$

There are three classes of generic extremal static black holes in this model. They all correspond to the same complex nilpotent orbit of $E_{7}(\mathbb{C})$, with weighted Dynkin diagram $[200000]$. We shall label them by their $\operatorname{Spin}^{*}(12) \times \mathrm{SL}(2, \mathbb{R})$ weighted Dynkin diagrams, which define both the real nilpotent orbit of $E_{7(-5)}$ in which their Noether charge lies, and the $\operatorname{Spin}^{*}(12) \times \mathrm{SL}(2, \mathbb{R})$ orbit in which the coset component of their Maurer-Cartan

\footnotetext{
${ }^{16}$ Note that after identifying $\rho_{0}=|Z|$, as we shall do in all $\mathcal{N}=2$ models, we can no longer assume that the canonical $\mathcal{N}=8$ ordering $\rho_{0} \geq \rho_{1} \geq \rho_{2} \geq \rho_{3}$ is satisfied, although we can still assume that $\rho_{1} \geq \rho_{2} \geq \rho_{3}$.
} 
form $P$ lies. 1/2-BPS black holes correspond to the weighted Dynkin diagram [00080.4], and are controlled by the fake superpotential $W=|Z|$. The non-BPS ones with $Z_{*}=0$ [02080.0] (which are 1/6-BPS in the context of $\mathcal{N}=6$ supergravity) are controlled by the fake superpotential $W=\rho_{1}\left(Z_{A B}\right)$, and the non-BPS black holes with $Z_{*} \neq 0\left[0000_{2 \cdot 2}\right]$, by the fake superpotential $W=2 \varrho\left(Z, Z_{A B}\right)$, which is the same function of $\rho_{0}, \rho_{1}, \rho_{2}, \rho_{3}$ and $\varphi$ as for the non-BPS black holes of maximal supergravity.

In addition to (3.1), there are three additional $\mathcal{N}=2$ 'magic' supergravity theories in $D=4$, associated to the octonions, complex and real numbers respectively. Their moduli spaces are given by [50]

$$
\begin{aligned}
& \text { o) } \mathcal{M}_{4} \cong\left(\mathrm{U}(1) \times E_{6(-78)}\right) \backslash E_{7(-25)}, \quad \mathcal{M}_{3}^{*} \cong\left(\mathrm{SL}(2) \times E_{7(-25)}\right) \backslash E_{8(-24)}, \\
& \text { c) } \mathcal{M}_{4} \cong S(\mathrm{U}(3) \times \mathrm{U}(3)) \backslash \mathrm{SU}(3,3), \quad \mathcal{M}_{3}^{*} \cong(\mathrm{SL}(2) \times \mathrm{SU}(3,3)) \backslash E_{6(2)} \text {, } \\
& r) \mathcal{M}_{4} \cong \mathrm{U}(3) \backslash S p(6, \mathbb{R}), \quad \quad \mathcal{M}_{3}^{*} \cong(\mathrm{SL}(2) \times \mathrm{Sp}(6, \mathbb{R})) \backslash F_{4(4)},
\end{aligned}
$$

respectively. The truncations from q) to c) and r) amount to restricting $Z_{A B}$ to the $\mathbf{3} \otimes \mathbf{3}$ of $S(\mathrm{U}(3) \times \mathrm{U}(3)) \subset \mathrm{SU}(6)$ and the symmetric tensor representation 6 of its diagonal subgroup $\mathrm{U}(3) \subset S(\mathrm{U}(3) \times \mathrm{U}(3))$, respectively. Case o) cannot be obtained by truncation from q), and needs to be discussed separately.

In this case, the electromagnetic charges transform in the $\mathbf{5 6}$ of $E_{7(-25)}$, which decomposes as $\mathbb{C} \oplus \mathbf{2 7}$ with respect to $\mathrm{U}(1) \times E_{6(-78)}$, with $\mathbf{2 7}$ being the complex fundamental representation of $E_{6(-78)}$. With respect to the $\mathrm{SU}(2) \times \mathrm{SU}(6) \subset E_{6(-78)}$ subgroup associated to the 'truncation to the quaternions', the complex $\mathbf{2 7}$ representation decomposes as $\mathbf{1 5} \oplus \mathbf{2} \otimes \mathbf{6}$, and the complex charge $Z_{a}$ splits this way into $Z_{A B}$ and $Z_{\alpha}^{A}$. The action of the remaining generators of $\mathfrak{e}_{6(-78)}$ in the $(\mathbf{2} \otimes \mathbf{2 0})_{\mathbb{R}}$ of $\mathrm{SU}(2) \times \mathrm{SU}(6)$, is defined as follows

$$
\delta Z_{A B}=\Lambda_{A B C}^{\alpha} Z_{\alpha}^{C}, \quad \delta Z_{\alpha}^{A}=-\Lambda_{\alpha}^{A B C} Z_{B C}
$$

in term of the complex self-dual parameters $\Lambda_{A B C}^{\alpha}=\frac{1}{6} \varepsilon^{\alpha \beta} \varepsilon_{A B C D E F} \Lambda_{\beta}^{D E F}$. Using these generators, one computes the quadratic and the cubic invariants ${ }^{17}$ of $E_{6(-78)}$ as

$$
\begin{aligned}
Z_{a} Z^{a} & =Z_{A B} Z^{A B}+Z_{\alpha}^{A} Z_{A}^{\alpha} \\
t^{a b c} Z_{a} Z_{b} Z_{c} & =3 \varepsilon^{\alpha \beta} Z_{A B} Z_{\alpha}^{A} Z_{\beta}^{B}+\frac{1}{4} \varepsilon^{A B C D E F} Z_{A B} Z_{C D} Z_{E F}
\end{aligned}
$$

A generic element $Z_{a}$ can always be rotated to a basis in which its component $Z_{\alpha}^{A}=0$, and $Z_{A B}$ takes a block diagonal form such that a generic charge $Z_{a}$ is parametrized by 50 angles of $\operatorname{Spin}(8) \backslash E_{6(-78)}$ and four invariants. The $\mathrm{SU}(6)$ invariant functions $\rho_{1}, \rho_{2}, \rho_{3}$ lift in a unique way to the roots of the $E_{6(-78)}$ invariant polynomial

$$
\begin{aligned}
\lambda^{3}-2 Z_{a} Z^{a} \lambda^{2}+2 t_{a b e} t^{c d e} Z_{c} Z_{d} Z^{a} Z^{b} \lambda-\frac{4}{9}\left|t^{a b c} Z_{a} Z_{b} Z_{c}\right|^{2} \\
=\left(\lambda-\rho_{1}{ }^{2}\right)\left(\lambda-\rho_{2}{ }^{2}\right)\left(\lambda-\rho_{3}{ }^{2}\right),
\end{aligned}
$$

\footnotetext{
${ }^{17}$ Do not confuse the $E_{6(-78)}$-invariant tensor $t^{a b c}$ appearing in (3.8) with the $E_{6(-26)}$-invariant tensor appearing in the standard cubic prepotential of $\mathcal{M}_{4}$.
} 
while $\rho_{0}$ and $\varphi$ are the $\mathrm{U}(1) \times E_{6(-78)}$ invariants

$$
\rho_{0}=[Z], \quad 4 \varphi=\arg \left[Z t^{a b c} Z_{a} Z_{b} Z_{c}\right] .
$$

In terms of these invariants, the $E_{7(-25)}$ quartic invariant is defined similarly to (2.15),

$$
\diamond(Z)=\left(|Z|^{2}-2 Z_{a} Z^{a}\right)^{2}-8 t_{a b e} t^{c d e} Z_{c} Z_{d} Z^{a} Z^{b}+\frac{16}{3} \operatorname{Re}\left[Z t^{a b c} Z_{a} Z_{b} Z_{c}\right]
$$

As in magic supergravity q) there are three classes of generic extremal black holes in this model, all of which correspond to the complex nilpotent orbit of weighted Dynkin diagram $[0000002]$ in $\mathfrak{e}_{8} \mathbb{C}$. We shall label them by their $E_{7(-25)} \times \mathrm{SL}(2, \mathbb{R})$ weighted Dynkin diagram. 1/2-BPS black holes, with weighted Dynkin diagram $[000000 \cdot 4]$, are controlled by the fake superpotential $W=|Z|$; non-BPS $Z_{*}=0$ black holes, with weighted Dynkin diagram $[200000.0]$, by the fake superpotential $W=\rho_{1}\left(Z_{a}\right)$; and the non-BPS $Z_{*} \neq 0$ black holes, with weighted Dynkin diagram $[.00002 .2]$, by the fake superpotential $W=2 \varrho\left(Z, Z_{a}\right)$. The latter is again the same function of $\rho_{0}, \rho_{1}, \rho_{2}, \rho_{3}$ and $\varphi$ as in $\mathcal{N}=8$ supergravity.

We will now explain how the fake superpotential controlling the non-BPS $Z_{*} \neq 0$ black holes can be derived from the parametrization of the nilpotent orbit of weighted Dynkin diagram $[000002.2]$. The latter tells us that the generator $\mathbf{h} \in \mathfrak{s l}_{2} \oplus \mathfrak{e}_{7(-25)}$ of the triplet associated to the nilpotent orbit defines the following five-graded decomposition of $\mathfrak{s l}_{2} \oplus \mathfrak{e}_{7(-25)}$ :

$$
\mathfrak{s l}_{2} \oplus \mathfrak{e}_{7(-25)} \cong(\mathbf{1} \oplus \mathbf{2 7})^{(-2)} \oplus\left(\mathfrak{g l}_{1} \oplus \mathfrak{g l}_{1} \oplus \mathfrak{e}_{6(-26)}\right)^{(0)} \oplus(\mathbf{1} \oplus \overline{\mathbf{2 7}})^{(2)}
$$

The corresponding decomposition of the coset component $\mathfrak{e}_{8(-24)} \ominus\left(\mathfrak{s l}_{2} \oplus \mathfrak{e}_{7(-25)}\right)$ is

$$
\mathbf{2} \otimes \mathbf{5 6} \cong \mathbf{1}^{(-4)} \oplus(\mathbf{1} \oplus \overline{\mathbf{2 7}})^{(-2)} \oplus(\mathbf{2 7} \oplus \overline{\mathbf{2 7}})^{(0)} \oplus(\mathbf{1} \oplus \mathbf{2 7})^{(2)} \oplus \mathbf{1}^{(4)},
$$

while the orbit of a representative $\mathbf{e} \in(\mathbf{1} \oplus \mathbf{2 7})^{(2)}$ associated to a regular black holes is ${ }^{18}$

$$
\begin{aligned}
\left(F_{4(-52)} \ltimes(\mathbf{1} \oplus \mathbf{2 6})^{(2)}\right) \backslash(\mathrm{SL}(2) & \left.\times E_{7(-25)}\right) \\
& \subset\left(E_{6(-26)} \ltimes\left((\mathbf{1} \oplus \mathbf{2 6} \oplus \overline{\mathbf{2 6}})^{(2)} \oplus \mathbf{1}^{(4)}\right)\right) \backslash E_{8(-24)} .
\end{aligned}
$$

The generator $\mathbf{h}$ is parametrized in this case by two phases $\alpha$ and $\beta$ and an element $\Omega_{a} \in F_{4(-52)} \backslash E_{6(-78)}$ satisfying ${ }^{19}$

$$
\Omega_{a}=-\frac{1}{2} t_{a b c} \Omega^{b} \Omega^{c}, \quad \Omega_{a} \Omega^{a}=6 .
$$

Let us consider for instance general charges $Z$ and $Z_{a}$, which we parametrize as

$$
Z=e^{-i(\alpha-3 \beta)}\left(\left(e^{2 i \alpha}+i \sin 2 \alpha\right) \varrho-\Omega^{a} \Xi_{a}\right), \quad Z_{a}=e^{-i(\alpha+\beta)}\left(\frac{1}{2}\left(e^{2 i \alpha}+i \sin 2 \alpha\right) \varrho \Omega_{a}+\Xi_{a}\right)
$$

\footnotetext{
${ }^{18}$ The representative of non-compact stabilizer in $E_{6(-26)}$ such as $F_{4(-20)}$ or $\operatorname{Spin}(9,1) \times \mathrm{SO}(1,1)$ are associated to black holes with naked singularities.

${ }^{19}$ which generalizes the symplectic form $\Omega_{A B} \in U S p(6) \backslash \mathrm{SU}(6)$ satisfying $\Omega_{A C} \Omega^{B C}=\delta_{A}^{B}$.
} 
where $\Xi_{a}$ lies in the $\mathbf{1} \oplus \mathbf{2 6}$ of the $F_{4(-52)} \subset E_{6(-78)}$ stabilizer of $\Omega_{a}$ defined as

$$
\Xi_{a}=\left(t_{a b c} \Omega^{c}+\frac{1}{2} \Omega_{a} \Omega_{b}\right) \Xi^{b}
$$

As in maximal supergravity, such decomposition is unique as long as the eigenvalues of $\Xi_{a}$ lie in the tetrahedron (2.70). To such electromagnetic charges, one associates a generator $\mathbf{h} \in \mathfrak{s l}_{2} \oplus \mathfrak{e}_{7(-25)}$ that acts on the elements of $P_{0}$ as follows

$$
\begin{aligned}
\mathbf{h} W & =\frac{1}{\cos 2 \alpha}\left(e^{i(\alpha-3 \beta)} Z+e^{i(\alpha+\beta)} \Omega^{a} Z_{a}\right)-4 i \tan 2 \alpha W \\
\mathbf{h} Z & =\frac{1}{\cos 2 \alpha}\left(e^{-i(\alpha-3 \beta)} W+e^{i(\alpha+\beta)} \Omega^{a} \Sigma_{a}\right)-2 i \tan 2 \alpha Z \\
\mathbf{h} Z_{a} & =\frac{1}{\cos 2 \alpha}\left(e^{i(\alpha-3 \beta)} \Sigma_{a}+\frac{1}{2} e^{-i(\alpha+\beta)} \Omega_{a} W+e^{i(\alpha+\beta)} t_{a b c} \Omega^{b} \Sigma^{c}\right)-2 i \tan 2 \alpha Z_{a} \\
\mathbf{h} \Sigma_{a} & =\frac{1}{\cos 2 \alpha}\left(e^{-i(\alpha-3 \beta)} Z_{a}+\frac{1}{2} e^{-i(\alpha+\beta)} \Omega_{a} Z+e^{i(\alpha+\beta)} t_{a b c} \Omega^{b} Z^{c}\right)
\end{aligned}
$$

The solution to the equation $\left[\mathbf{h}, P_{0}\right]=2 P_{0}$ is then

$$
W=2 \varrho, \quad \Sigma_{a}=e^{2 i \beta}\left(i \sin 2 \alpha \varrho \Omega_{a}+\Xi_{a}-\frac{1}{2} \Omega_{a} \Omega^{b} \Xi_{b}\right)
$$

As for the maximally supersymmetric case one shows that the equation $[\mathbf{h}, P]=2 P$ is equivalent to a gradient flow with respect to the fake superpotential $W=2 \varrho\left(Z, Z_{a}\right)$. The only non-trivial step compared to $(2.43),(2.79)$ is to check that the $\mathfrak{e}_{6(-78)}$ generators acting on $\Omega_{a}$, give an element satisfying

$$
\Lambda_{a}^{b} \Omega_{b}=-\left(t_{a b c} \Omega^{c}+\frac{1}{2} \Omega_{a} \Omega_{b}\right) \Lambda_{d}^{b} \Omega^{d},
$$

generalizing (2.82), (2.83), (2.84), such that only the coset component of the scalar derivative contributes.

From (3.19), it is easily seen that the flat directions of the fake superpotential span the $\mathbf{2 6}$ real representation of $F_{4(-52)}$ inside the complex $\mathbf{2 7}$ of $E_{6(-78)}$. The maximum of $W$ correspond to $\alpha=\Xi_{a}=0$, which gives rise to a manifold of flat directions

$$
\mathcal{M}_{\text {non-BPS }} \cong F_{4(-52)} \backslash E_{6(-26)},
$$

isomorphic to the moduli space after decompactification to five dimensions. At the attractor point, $\diamond\left(Z_{*}, Z_{* a}\right)=-W_{*}^{4}$ and one recovers the familiar expression of the entropy for nonBPS black holes with $Z_{*} \neq 0$,

$$
S_{\mathrm{BH}}=\pi \sqrt{-\diamond(p, q)} .
$$

It is rather remarkable that the nilpotent orbits of the Lie algebras $\mathfrak{f}_{4(4)}, \mathfrak{e}_{6(2)}, \mathfrak{e}_{7(-5)}$ and $\mathfrak{e}_{8(-24)}$ associated to the real numbers, complex numbers, quaternions and octonions, respectively, satisfying the nilpotency conditions

$$
\left[\mathbf{e}_{\mid \mathbf{2 6}}\right]^{3}=0, \quad\left[\mathbf{e}_{\mid \mathbf{2 7}}\right]^{3}=0, \quad\left[\mathbf{e}_{\mid \mathbf{5 6}}\right]^{3}=0, \quad\left[\mathbf{e}_{\mid \mathbf{3 8 7 5}}\right]^{5}=0,
$$




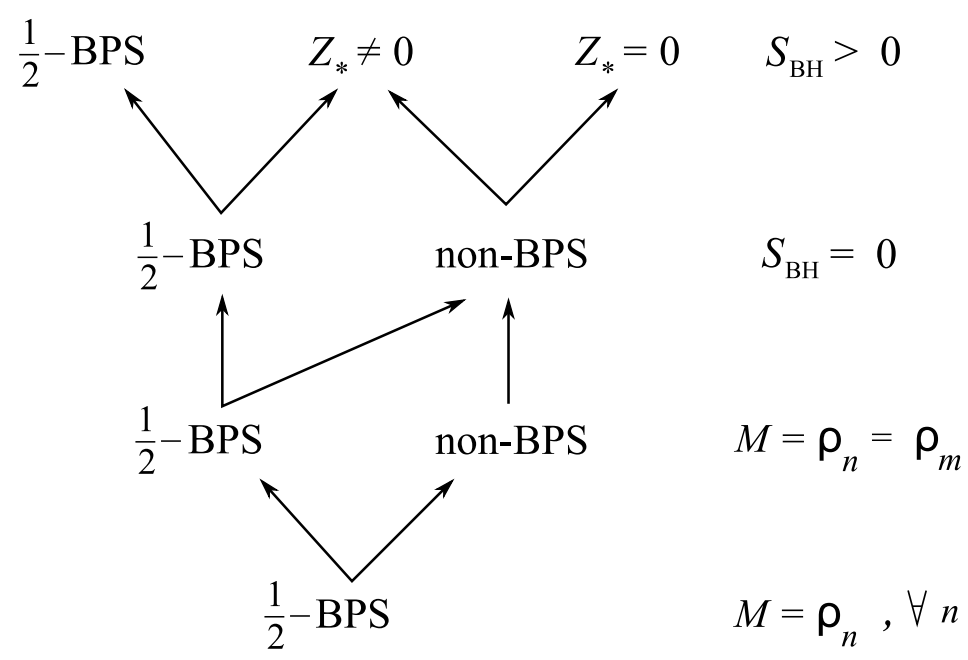

Figure 2. Stratification of the moduli space of extremal solutions for magic supergravity models.

are in one-to-one correspondence [51-54],

$$
\begin{aligned}
F_{4(4)} \backslash\left\{\mathbf{e} \in \mathfrak{f}_{4(4)} \mid\left[\mathbf{e}_{\mid \mathbf{2 6}}\right]^{3}=0\right\} \cong E_{6(2)} \backslash\left\{\mathbf{e} \in \mathfrak{e}_{6(2)} \mid\left[\mathbf{e}_{\mid \mathbf{2 7}}\right]^{3}=0\right\} \\
\cong E_{7(-5)} \backslash\left\{\mathbf{e} \in \mathfrak{e}_{7(-5)} \mid\left[\mathbf{e}_{\mid \mathbf{5 6}}\right]^{3}=0\right\} \cong E_{8(-24)} \backslash\left\{\mathbf{e} \in \mathfrak{e}_{8(-24)} \mid\left[\mathbf{e}_{\mid \mathbf{3 8 7 5}}\right]^{5}=0\right\},
\end{aligned}
$$

so that the corresponding moduli space of extremal black holes have the same stratified structure in term of $K_{3}^{*}$ orbits, which is displayed in figure 2 .

\subsection{Axion-dilaton $\mathcal{N}=2$ supergravity}

The quaternionic magic supergravity (3.1) can also be further truncated by singling out an additional complex charge $Z_{1}=2 Z_{34}$ from $Z_{A B}$, such that the internal symmetry group is restricted to $\mathrm{U}(2) \times \mathrm{U}(4) \subset \mathrm{U}(6)$. The remaining charges transform as a complex vector of $\mathrm{SO}(6) \cong \mathbb{Z}_{2} \backslash \mathrm{SU}(4)$ and the scalar fields parametrize the symmetric spaces

$$
\begin{aligned}
& \mathcal{M}_{4} \cong \mathrm{U}(1) \backslash \mathrm{SL}(2, \mathbb{R}) \times(\mathrm{SO}(2) \times \mathrm{SO}(6)) \backslash \mathrm{SO}(2,6), \\
& \mathcal{M}_{3}^{*} \cong(\mathrm{SO}(2,2) \times \mathrm{SO}(2,6)) \backslash \mathrm{SO}(4,8) .
\end{aligned}
$$

More generally, one obtains this way all $\mathcal{N}=2$ supergravity theories with $n+1$ vector multiplets $(0<n \leq 6)$ coupled to scalar fields parametrising the symmetric space

$$
\begin{aligned}
& \mathcal{M}_{4} \cong \mathrm{U}(1) \backslash \mathrm{SL}(2, \mathbb{R}) \times(\mathrm{SO}(2) \times \mathrm{SO}(n)) \backslash \mathrm{SO}(2, n), \\
& \mathcal{M}_{3}^{*} \cong(\mathrm{SO}(2,2) \times \mathrm{SO}(2, n)) \backslash \mathrm{SO}(4, n+2) .
\end{aligned}
$$

Denoting the $n$ charges transforming in the vector representation of $\mathrm{SO}(n)$ as $Z_{\mathrm{i}}$, one can identify

$$
\rho_{0}=|Z|, \quad \rho_{1}=\left|Z_{1}\right|, \quad 4 \varphi=\arg \left[Z \bar{Z}_{1} \bar{Z}^{\mathrm{i}} \bar{Z}_{\mathrm{i}}\right]
$$


while $\rho_{2}$ and $\rho_{3}$ follow from the roots of the polynomial ${ }^{20}$

$$
\lambda^{2}-Z^{\mathrm{i}} \bar{Z}_{\mathrm{i}} \lambda+\frac{1}{4}\left|Z^{\mathrm{i}} Z_{\mathrm{i}}\right|^{2}=\left(\lambda-\rho_{2}{ }^{2}\right)\left(\lambda-\rho_{3}{ }^{2}\right) .
$$

For $n>2$ there are four $\mathrm{SL}(2, \mathbb{R}) \times \mathrm{SL}(2, \mathbb{R}) \times \mathrm{SO}(2, n)$ orbits of generic extremal black holes associated to four distinct nilpotent orbits of $\mathfrak{s o}(4,2+n)$ of degree three in the spinor representation (i.e. such that $\mathbf{e}^{3}=0$ and $\operatorname{ad}_{\mathbf{e}}{ }^{4} \neq 0$ ). For $1 / 2$-BPS black holes, $W=|Z|$; for non-BPS black holes with $Z_{*}=Z_{*}^{\mathrm{i}}=0, W=\left|Z_{1}\right|$; for the non-BPS black holes with $Z_{*}=Z_{1 *}=0$,

$$
W=\sqrt{\frac{1}{2} Z^{\mathrm{i}} \bar{Z}_{\mathrm{i}}+\frac{1}{2} \sqrt{\left(Z^{\mathrm{i}} \bar{Z}_{\mathrm{i}}\right)^{2}-\left|Z^{\mathrm{i}} Z_{\mathrm{i}}\right|^{2}}}
$$

and finally, for non-BPS black holes with $Z_{*} \neq 0, W=2 \varrho$ with the same function $\varrho$ as in $\mathcal{N}=8$ supergravity. Of course these results extend straightforwardly to any value of $n$ larger than six.

The STU model, to be expanded upon in section 4 , corresponds to $n=2$. The STU truncation of maximal supergravity is defined by a basis such that

$$
Z_{i j} \hat{=} \frac{1}{2}\left(\begin{array}{cc}
0 & 1 \\
-1 & 0
\end{array}\right) \otimes\left(\begin{array}{cccc}
Z & 0 & 0 & 0 \\
0 & \bar{Z}_{1} & 0 & 0 \\
0 & 0 & \bar{Z}_{2} & 0 \\
0 & 0 & 0 & \bar{Z}_{3}
\end{array}\right)
$$

which is preserved by a subgroup $S(\mathrm{U}(2) \times \mathrm{U}(2) \times \mathrm{U}(2) \times \mathrm{U}(2)) \subset \mathrm{SU}(8)$ as well as

$$
[\mathrm{SL}(2, \mathbb{R})]^{3} \times[\mathrm{SU}(2)]^{4} \subset E_{7(7)}
$$

The SU(2) subgroups leave invariant the bosonic fields and will be disregarded. Considering the whole field content of the STU model, only one SU(2) factor acts non-trivially on the fermionic fields, and corresponds to the R-symmetry group. There are five different types of extremal static black holes in this model. Four of them, including the 1/2-BPS ones, correspond to $1 / 8$-BPS black holes within $\mathcal{N}=8$ supergravity. Their fake superpotential is then $W=|Z|,\left|Z_{1}\right|,\left|Z_{2}\right|,\left|Z_{3}\right|$, respectively. The last type corresponds to the non-BPS black holes of $\mathcal{N}=8$ supergravity; its fake superpotential is obtained from $W=2 \varrho$ by substituting $|Z|,\left|Z_{1}\right|,\left|Z_{2}\right|,\left|Z_{3}\right|$ and $\frac{1}{4} \arg \left(Z \bar{Z}_{1} \bar{Z}_{2} \bar{Z}_{3}\right)$ to $\rho_{0}, \rho_{1}, \rho_{2}, \rho_{3}$ and $\varphi$.

There are two interesting further truncations, which are obtained by restriction to the diagonal subgroup of either two $\operatorname{SL}(2, \mathbb{R})$ factors or the three of them. In the first case one obtains the $S^{2} T$ model (i.e. (3.26) with $n=1$ ). It admits three types of extremal static black holes, the $1 / 2$-BPS ones, for which $W=|Z|$; the non-BPS ones with $Z_{*}=0$, for which $W=\left|Z_{1}\right|$; and the non-BPS ones with $Z_{*} \neq 0$, for which $W=2 \varrho\left(Z, Z_{1}, Z_{2}\right)$. As explained in section B.3, the degree six polynomial (B.14) factorizes and an explicit expression of $W$ in terms of $Z, Z_{1}$ and $Z_{2}$ can be obtained by solving the quartic polynomial (B.20).

The subsequent truncation is the $S^{3}$ model, which is the circle compactification of the minimal $\mathcal{N}=2$ supergravity theory in five dimensions.

$$
\mathcal{M}_{4} \cong \mathrm{U}(1) \backslash \mathrm{SL}(2, \mathbb{R}), \quad \mathcal{M}_{3}^{*} \cong \mathrm{SO}(2,2) \backslash G_{2(2)}
$$

\footnotetext{
${ }^{20}$ Note that raising the $\mathrm{SO}(n)$ index i does not involve complex conjugation.
} 


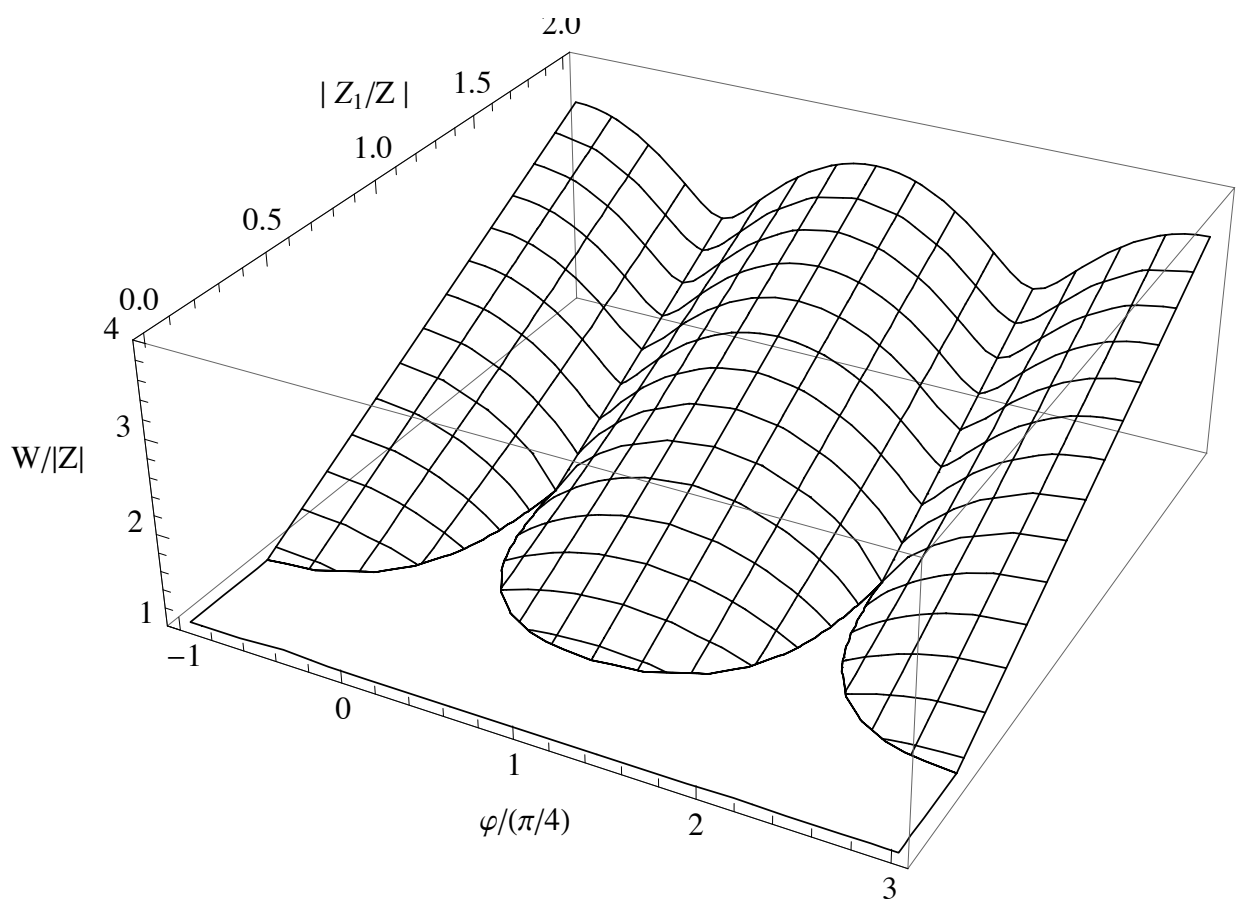

Figure 3. Normalized fake superpotential $W /|Z|$ as a function of $\left|Z_{1} / Z\right|$ and $\varphi /(\pi / 4)$.

This theory admits two types of extremal black holes, the 1/2-BPS ones for which $W=|Z|$, and the non-BPS ones with $Z_{*} \neq 0$, corresponding to the two nilpotent orbits of $\mathfrak{g}_{2(2)}$ in which $\left[\mathbf{e}_{\mid 7}\right]^{3}=0[20]$. As explained in B.4, the degree six polynomial (B.14) reduces to a cubic polynomial (B.22). Identifying $\rho_{0}=|Z|$ and $\rho_{1}=\left|Z_{1}\right|$ where $Z_{1}=-2 i S_{2} D Z$, one obtains an algebraic expression for the fake superpotential,

$$
W=\frac{1}{2} \sqrt{|Z|^{2}+3\left(\left|Z_{1}\right|^{2}+L_{+}+L_{-}\right)},
$$

where $L_{ \pm}$are given by (B.28) below, which we rewrite for convenience,

$$
L_{ \pm}^{3}=\left|Z_{1}\right|^{4}\left(\left|Z_{1}\right|^{2}+3|Z|^{2}\right)-\frac{1}{2}\left(Z \bar{Z}_{1}^{3}+\bar{Z} Z_{1}^{3}\right)\left(|Z|^{2}+3\left|Z_{1}\right|^{2}\right) \pm \frac{\sqrt{-\downarrow}}{2}\left|Z \bar{Z}_{1}^{3}-\bar{Z} Z_{1}^{3}\right|,
$$

and $\diamond$ is the moduli-independent quartic $\mathrm{SL}(2, \mathbb{R})$ invariant

$$
\diamond\left(Z, Z_{1}\right)=\left(|Z|^{2}-\left|Z_{1}\right|^{2}\right)^{2}-4\left|Z \bar{Z}_{1}-Z_{1}^{2}\right|^{2}
$$

The fake superpotential $W$ in (3.33) is plotted as a function of $\varphi$ in figure 3. Contrary to the superpotential for BPS black holes, which is $\varphi$ independent, $W$ is maximal at $\varphi=\pi / 2\left(n+\frac{1}{2}\right), n \in \mathbb{Z}$. Note that (3.33) is only valid in the region where $\diamond<0$, such that $W>|Z|$. In the region where $\diamond>0$ it must be replaced by the BPS superpotential $W=|Z|$.

\section{$3.3 \mathcal{N}=4$ supergravity}

$\mathcal{N}=8$ supergravity can be truncated to $\mathcal{N}=4$ supergravity coupled to $n=6$ vector multiplets by restricting the central charges to two antisymmetric tensors of $\mathrm{SU}(4) \times \mathrm{SU}(4)$ 
such that $S(\mathrm{U}(4) \times \mathrm{U}(4)) \subset \mathrm{SU}(8)$. The central charges $Z_{A B}$ are then defined as an antisymmetric tensor of the first $\mathrm{SU}(4)$ that we shall identify as the R-symmetry group of the theory. For further generalization, it will be more convenient to represent the other six complex electromagnetic charges as a complex vector $Z^{\mathrm{i}}$ of $\mathrm{SO}(n) \cong \mathbb{Z}_{2} \backslash \mathrm{SU}(4)$ for $n=6$. Then, the $\mathrm{SU}(8)$ invariant functions become $S(\mathrm{U}(4) \times \mathrm{U}(4))$ invariant functions defined by the two polynomials

$$
\begin{aligned}
& \left(\lambda-\rho_{0}^{2}\right)\left(\lambda-\rho_{1}^{2}\right)=\lambda^{2}-2 Z_{A B} Z^{A B} \lambda+2\left(\left(Z_{A B} Z^{A B}\right)^{2}-2 Z_{A B} Z^{B C} Z_{C D} Z^{D A}\right) \\
& \left(\lambda-\rho_{2}^{2}\right)\left(\lambda-\rho_{3}^{2}\right)=\lambda^{2}-Z^{\mathrm{i}} \bar{Z}_{\mathrm{i}} \lambda+\frac{1}{4}\left|Z^{\mathrm{i}} Z_{\mathrm{i}}\right|^{2}
\end{aligned}
$$

and

$$
4 \varphi=\arg \left[\varepsilon^{A B C D} Z_{A B} Z_{C D} \bar{Z}_{\mathrm{i}} \bar{Z}^{\mathrm{i}}\right] .
$$

This model admits three types of generic extremal black holes, which are in one-to-one correspondence with the nilpotent orbits of $\mathfrak{s o}(8,8)$ of degree three in the spinor representation [24] (i.e. such that $\mathbf{e}^{3}=0$ and $\operatorname{ad}_{\mathbf{e}}{ }^{4} \neq 0$ ). They are: the $1 / 4$-BPS black holes, for which

$$
W=\sqrt{Z_{A B} Z^{A B}+\sqrt{4 Z_{A B} Z^{B C} Z_{C D} Z^{D A}-\left(Z_{A B} Z^{A B}\right)^{2}}}
$$

the non-BPS black holes with $Z_{* A B}=0$, for which

$$
W=\sqrt{\frac{1}{2} Z^{\mathrm{i}} \bar{Z}_{\mathrm{i}}+\frac{1}{2} \sqrt{\left(Z^{\mathrm{i}} \bar{Z}_{\mathrm{i}}\right)^{2}-\left|Z^{\mathrm{i}} Z_{\mathrm{i}}\right|^{2}}}
$$

and the non-BPS ones with $Z_{* A B} \neq 0$, for which the fake superpotential is the same function $2 \varrho\left(Z_{A B}, Z_{\mathrm{i}}\right)$ as in $\mathcal{N}=8$ supergravity. These results extend straightforwardly to $\mathcal{N}=4$ supergravity coupled to any number $n>2$ of vector multiplets, with moduli space

$$
\begin{aligned}
& \mathcal{M}_{4} \cong \mathrm{U}(1) \backslash \mathrm{SL}(2, \mathbb{R}) \times(\mathrm{SO}(6) \times \mathrm{SO}(n)) \backslash \mathrm{SO}(6, n), \\
& \mathcal{M}_{3}^{*} \cong(\mathrm{SO}(6,2) \times \mathrm{SO}(2, n)) \backslash \mathrm{SO}(8, n+2) .
\end{aligned}
$$

Non-BPS multi-black hole solutions with $Z_{* A B} \neq 0$ have been discussed in [26].

For $n=2$, the scalar moduli space (3.41) of the $\mathcal{N}=4$ model becomes identical to that of the $\mathcal{N}=2$ model (3.26) with $n=6$. The nilpotent orbit associated to the non-BPS black holes with $Z_{* A B}=0$ splits into two inequivalent orbits of the connected component of $\mathrm{SO}(8,4)$, giving rise to two inequivalent $\mathrm{SO}(6,2) \times \mathrm{SL}(2, \mathbb{R}) \times \mathrm{SL}(2, \mathbb{R})$ orbits . With only one vector multiplet, there is no non-BPS black holes with $Z_{* A B}=0$. The degree six polynomial defining the fake superpotential $W=2 \varrho$ associated to non-BPS black holes with $Z_{* A B} \neq 0$ reduces to the degree four polynomial (B.20) in such a way that the explicit form of $W$ can then be derived straightforwardly.

\subsection{Axion-dilaton gravity}

The non-standard diagonalization problem defining $\varrho(Z)$ can in fact be formulated more generally for the case of Einstein gravity coupled to $m+n$ abelian vector fields and to 
scalars valued in the symmetric spaces (with $m \geq 1, n \geq 1$ )

$$
\begin{aligned}
& \mathcal{M}_{4} \cong \mathrm{U}(1) \backslash \mathrm{SL}(2, \mathbb{R}) \times(\mathrm{SO}(m) \times \mathrm{SO}(n)) \backslash \mathrm{SO}(m, n), \\
& \mathcal{M}_{3}^{*} \cong(\mathrm{SO}(m, 2) \times \mathrm{SO}(2, n)) \backslash \mathrm{SO}(m+2, n+2) .
\end{aligned}
$$

For $m=2$ and $m=6$, respectively one recovers the cases of axion-dilaton $\mathcal{N}=2$ supergravities and $\mathcal{N}=4$ supergravities discussed previously. Denoting the $\mathrm{SO}(m)$ and $\mathrm{SO}(n)$ vectors of charges by $Z_{\mathrm{a}}$ and $Z_{\mathrm{i}}$, respectively, ${ }^{21}$ the non-standard diagonalization problem can be formulated in term of two unit-norm vectors $\Omega_{\mathrm{a}}$ and $\Omega_{\mathrm{i}}$ as follows,

$$
\begin{aligned}
& Z_{\mathrm{a}}=e^{-i(\alpha-\beta)}\left(\left(e^{2 i \alpha}+i \sin 2 \alpha\right) \varrho \Omega_{\mathrm{a}}+\xi \Omega_{\mathrm{a}}+i \Xi_{\mathrm{a}}\right), \\
& \bar{Z}_{\mathrm{i}}=e^{-i(\alpha+\beta)}\left(-i\left(e^{2 i \alpha}+i \sin 2 \alpha\right) \varrho \Omega_{\mathrm{i}}+i \xi \Omega_{\mathrm{i}}+\Xi_{\mathrm{i}}\right),
\end{aligned}
$$

where $\Xi_{\mathrm{a}}$ and $\Xi_{\mathrm{i}}$ are real vectors orthogonal to $\Omega_{\mathrm{a}}$ and $\Omega_{\mathrm{i}}$, respectively.

We use similar notations as we did in exceptional $\mathcal{N}=2$ supergravity, i.e. $W=M+i k$, $\Sigma$ denotes the complex scalar charge associated to $\mathfrak{s l}_{2}$, and $\sigma_{\text {ai }}$ denote the real scalar charges associated to $\mathfrak{s o}(m, n)$. The nilpotent orbit is characterized by the generator $\mathbf{h}$ of $\mathfrak{s o}(m, 2) \oplus \mathfrak{s o}(2, n)$, which acts as follows

$$
\begin{aligned}
\mathbf{h} W & =\frac{2}{\cos 2 \alpha}\left(e^{i(\alpha-\beta)} \Omega^{\mathrm{a}} Z_{\mathrm{a}}+i e^{i(\alpha+\beta)} \Omega^{\mathrm{i}} \bar{Z}_{\mathrm{i}}\right)-4 i \tan 2 \alpha W \\
\mathbf{h} Z_{\mathrm{a}} & =\frac{1}{\cos 2 \alpha}\left(e^{-i(\alpha-\beta)} \Omega_{\mathrm{a}} W+e^{i(\alpha-\beta)} \Omega_{\mathrm{a}} \Sigma+2 i e^{i(\alpha+\beta)} \Omega^{\mathrm{i}} \sigma_{\mathrm{ai}}\right)-2 i \tan 2 \alpha Z_{\mathrm{a}} \\
\mathbf{h} \bar{Z}_{\mathrm{i}} & =\frac{1}{\cos 2 \alpha}\left(i e^{i(\alpha+\beta)} \Omega_{\mathrm{i}} \bar{\Sigma}-i e^{-i(\alpha+\beta)} \Omega_{\mathrm{i}} W+2 e^{i(\alpha-\beta)} \Omega^{\mathrm{a}} \sigma_{\mathrm{ai}}\right)-2 i \tan 2 \alpha \bar{Z}_{\mathrm{i}} \\
\mathbf{h} \Sigma & =\frac{2}{\cos 2 \alpha}\left(e^{-i(\alpha-\beta)} \Omega^{\mathrm{a}} Z_{\mathrm{a}}+i e^{i(\alpha+\beta)} \Omega^{\mathrm{i}} Z_{\mathrm{i}}\right) \\
\mathbf{h} \sigma_{\mathrm{ai}} & =\frac{2}{\cos 2 \alpha} \operatorname{Re}\left(e^{-i(\alpha-\beta)} \Omega_{\mathrm{a}} \bar{Z}_{\mathrm{i}}-i e^{-i(\alpha+\beta)} \Omega_{\mathrm{i}} Z_{\mathrm{a}}\right)
\end{aligned}
$$

The solution to the equation $\mathbf{h} P_{0}=2 P_{0}$ is then

$$
W=2 \varrho, \quad \Sigma=2 e^{2 i \beta}(\xi+i \sin (2 \alpha) \varrho), \quad \sigma_{\mathrm{a} i}=\Omega_{\mathrm{a}} \Xi_{\mathrm{i}}+\Omega_{\mathrm{i}} \Xi_{\mathrm{a}}+2 \sin (2 \alpha) \varrho \Omega_{\mathrm{a}} \Omega_{\mathrm{i}} .
$$

As before, the linear equation $\mathbf{h} P=2 P$ is equivalent to a gradient flow with respect to the fake superpotential $W=2 \varrho\left(Z_{\mathrm{a}}, Z_{\mathrm{i}}\right)$. For $m \leq 6, n \leq 6, W$ is necessarily the same function of the five $\mathrm{SO}(2) \times \mathrm{SO}(m) \times \mathrm{SO}(n)$ invariants

$$
\begin{aligned}
& \left(\lambda-\rho_{0}{ }^{2}\right)\left(\lambda-\rho_{1}{ }^{2}\right)=\lambda^{2}-Z^{\mathrm{a}} \bar{Z}_{\mathrm{a}} \lambda+\frac{1}{4}\left|Z^{\mathrm{a}} Z_{\mathrm{a}}\right|^{2}, \\
& \left(\lambda-\rho_{2}{ }^{2}\right)\left(\lambda-{\rho_{3}}^{2}\right)=\lambda^{2}-Z^{\mathrm{i}} \bar{Z}_{\mathrm{i}} \lambda+\frac{1}{4}\left|Z^{\mathrm{i}} Z_{\mathrm{i}}\right|^{2},
\end{aligned}
$$

and

$$
4 \varphi=\arg \left[Z_{\mathrm{a}} Z^{\mathrm{a}} \bar{Z}_{\mathrm{i}} \bar{Z}^{\mathrm{i}}\right]
$$

\footnotetext{
${ }^{21}$ For $\mathcal{N}=2, Z_{\mathrm{a}}$ is $Z$ for a $=1$ and $\bar{Z}_{1}$ for a $=2$. For $\mathcal{N}=4, Z_{\mathrm{a}}\left[\gamma^{\mathrm{a}}\right]_{A B}=Z_{A B}$, where the $\gamma^{\mathrm{a}}$ s are Dirac matrices of $\operatorname{Spin}(6)$.
} 
as in $\mathcal{N}=8$ supergravity, as it can be obtained by consistent truncation, and therefore remains so for any values $m$ and $n$.

From (3.44) it is apparent that the flat directions of $W$ are the components of $\sigma_{\mathrm{ai}}$ orthogonal to both $\Omega_{\mathrm{a}}$ and $\Omega_{\mathrm{i}}$, as well as the component $\Omega^{\mathrm{a}} \Omega^{\mathrm{i}} \sigma_{\mathrm{ai}}-\operatorname{Im}\left(e^{-2 i \beta} \Sigma\right)$, which altogether generate the expected symmetric space

$$
\mathcal{M}_{5} \cong G L(1, \mathbb{R}) \times(\mathrm{SO}(m-1) \times \mathrm{SO}(n-1)) \backslash \mathrm{SO}(m-1, n-1)
$$

The subgroup $G L(1, \mathbb{R}) \times \mathrm{SO}(m-1, n-1) \subset \mathrm{SL}(2, \mathbb{R}) \times \mathrm{SO}(m, n)$ is also the stabilizer of the charges at the horizon (at $\tau \rightarrow+\infty$ ), where they are of the form

$$
Z_{* \mathrm{a}}=\frac{1}{2} e^{i \beta_{*}} W_{*} \Omega_{* \mathrm{a}}, \quad Z_{* \mathrm{i}}=\frac{i}{2} e^{i \beta_{*}} W_{*} \Omega_{* \mathrm{i}} .
$$

\section{Extremal black holes in the $S T U$ model}

In this section, we expand the previous results in the case of the $S T U$ model of $\mathcal{N}=2$ supergravity, which generates all other symmetric models by truncation or covariantization. In particular, we interpret the BPS and non-BPS, $Z_{*}=0$ solutions in terms of the para-quaternionic structure on $\mathcal{M}_{3}^{*}$, and confirm our identification of nilpotent orbits on explicit solutions.

\subsection{Moduli spaces in $D=4$ and $D=3$}

The STU model of $\mathcal{N}=2$ supergravity is governed by the prepotential

$$
F=-X^{1} X^{2} X^{3} / X^{0}, \quad S=\frac{X^{1}}{X^{0}}, \quad T=\frac{X^{2}}{X^{0}}, \quad U=\frac{X^{3}}{X^{0}},
$$

The 4D moduli space consists of 3 copies of the Poincaré upper half plane, with metric ${ }^{22}$

$$
d s_{\mathcal{M}_{4}}^{2}=\frac{d S_{1}^{2}+d S_{2}^{2}}{S_{2}^{2}}+\frac{d T_{1}^{2}+d T_{2}^{2}}{T_{2}^{2}}+\frac{d U_{1}^{2}+d U_{2}^{2}}{U_{2}^{2}},
$$

and so is the symmetric space $\mathcal{M}_{4}=[\mathrm{U}(1) \backslash \mathrm{SL}(2, \mathbb{R})]^{3}$. For conciseness we shall denote $(S, T, U)=\left(S^{(1)}, S^{(2)}, S^{(3)}\right)$, while $S_{1}^{(i)}$ and $S_{2}^{(i)}$ will denote the real and imaginary part of $S^{(1)}$.

Upon dimensional reduction along a space-like direction, the resulting moduli space in 3 dimensions is the c-map of $\mathcal{M}_{4}$,

$$
\begin{aligned}
d s_{\mathcal{M}_{3}}^{2}= & d \phi^{2}+d s_{\mathcal{M}_{4}}^{2}+\frac{1}{4} e^{-2 \phi}\left(d \sigma+\tilde{\zeta}^{\Lambda} d \zeta_{\Lambda}-\zeta^{\Lambda} d \tilde{\zeta}_{\Lambda}\right)^{2} \\
& -e^{-\phi}\left[\left(d \tilde{\zeta}_{\Lambda}+\operatorname{Re} \mathcal{N}_{\Lambda \Sigma} \zeta^{\Sigma}\right)[\operatorname{Im} \mathcal{N}]^{\Lambda \Lambda^{\prime}}\left(d \tilde{\zeta}_{\Lambda^{\prime}}+\operatorname{Re} \mathcal{N}_{\Lambda^{\prime} \Sigma} \zeta^{\Sigma}\right)+d \zeta^{\Lambda}[\operatorname{Im} \mathcal{N}]_{\Lambda \Lambda^{\prime}} d \zeta^{\Lambda^{\prime}}\right]
\end{aligned}
$$

\footnotetext{
${ }^{22}$ In this section, to avoid notational conflict, we rename the variable $U$ appearing in the metric ansatz (1.2) into $\phi / 2$.
} 
(recall that $\operatorname{Im} \mathcal{N}$ is definite negative, so the metric on $\mathcal{M}_{3}$ is definite positive). Upon dimensional reduction along a time-like direction, the resulting moduli space in 3 dimensions is instead the pseudo-Riemannian space

$$
\begin{aligned}
d s_{\mathcal{M}_{3}^{*}}^{2}= & d \phi^{2}+d s_{\mathcal{M}_{4}}^{2}+\frac{1}{4} e^{-2 \phi}\left(d \sigma+\tilde{\zeta}^{\Lambda} d \zeta_{\Lambda}-\zeta^{\Lambda} d \tilde{\zeta}_{\Lambda}\right)^{2} \\
& +\frac{1}{2} e^{-\phi}\left[\left(d \tilde{\zeta}_{\Lambda}+\operatorname{Re} \mathcal{N}_{\Lambda \Sigma} \zeta^{\Sigma}\right)[\operatorname{Im} \mathcal{N}]^{\Lambda \Lambda^{\prime}}\left(d \tilde{\zeta}_{\Lambda^{\prime}}+\operatorname{Re} \mathcal{N}_{\Lambda^{\prime} \Sigma} \zeta^{\Sigma}\right)+d \zeta^{\Lambda}[\operatorname{Im} \mathcal{N}]_{\Lambda \Lambda^{\prime}} d \zeta^{\Lambda^{\prime}}\right]
\end{aligned}
$$

obtained from the Riemannian space (4.3) by analytic continuation $\zeta^{\Lambda} \rightarrow i \zeta^{\Lambda}, \tilde{\zeta}_{\Lambda} \rightarrow$ $i \tilde{\zeta}_{\Lambda}, \sigma \rightarrow-\sigma$.

Both $\mathcal{M}_{3}$ and $\mathcal{M}_{3}^{*}$ are symmetric spaces for the group $G_{3}=\mathrm{SO}(4,4)$,

$$
\mathcal{M}_{3}=[\mathrm{SO}(4)]^{2} \backslash \mathrm{SO}(4,4), \quad \mathcal{M}_{3}^{*}=[\mathrm{SO}(2,2)]^{2} \backslash \mathrm{SO}(4,4) .
$$

Note that in the second case, the denominator is not a maximal compact subgroup of $G_{3}$, which accounts for the signature $(8,8)$ of the metric on $\mathcal{M}_{3}^{*}$. To check that the metrics $(4.3)$ and (4.4) are the right-invariant metrics on the two symmetric spaces (4.5), it is convenient to choose an explicit parametrization of the Lie algebra $\mathfrak{g}_{3}=\mathfrak{s o}(4,4) \ni X=\sum \underline{E}_{a} E_{a}$, where $E_{a}$ are the 28 generators and $\underline{E}_{a}$ are the 28 dual coordinates,

$$
X=\left(\begin{array}{cccccccc}
\underline{H}_{2}+\underline{H}_{3} & -\underline{E}_{3} & -\underline{F}_{q_{1}} & \underline{F}_{q_{0}} & 0 & -\underline{E}_{2} & \underline{E}_{p^{0}} & \underline{E}_{p^{1}} \\
-\underline{F}_{3} & \underline{H}_{2}-\underline{H}_{3} & -\underline{F}_{p^{2}} & \underline{F}_{q_{3}} & \underline{E}_{2} & 0 & \underline{E}_{p^{3}} & -\underline{E}_{q_{2}} \\
-\underline{E}_{q_{1}} & -\underline{E}_{p^{2}} & \underline{H}+\underline{H}_{1} & -\underline{E}_{1} & -\underline{E}_{p^{0}} & -\underline{E}_{p^{3}} & 0 & -\underline{E}_{0} \\
\underline{E}_{q_{0}} & \underline{E}_{q_{3}} & -\underline{F}_{1} & \underline{H}-\underline{H}_{1} & -\underline{E}_{p^{1}} & \underline{E}_{q_{2}} & \underline{E}_{0} & 0 \\
0 & \underline{F}_{2} & -\underline{F}_{p^{0}} & -\underline{F}_{p^{1}} & -\underline{H}_{2}-\underline{H}_{3} & \underline{F}_{3} & \underline{E}_{q_{1}} & -\underline{E}_{q_{0}} \\
-\underline{F}_{2} & 0 & -\underline{F}_{p^{3}} & \underline{F}_{q_{2}} & \underline{E}_{3} & \underline{H}_{3}-\underline{H}_{2} & \underline{E}_{p^{2}} & -\underline{E}_{q_{3}} \\
\underline{F}_{p^{0}} & \underline{F}_{p^{3}} & 0 & \underline{F}_{0} & \underline{F}_{q_{1}} & \underline{F}_{p^{2}} & -\underline{H}-\underline{H}_{1} & \underline{F}_{1} \\
\underline{F}_{p^{1}} & -\underline{F}_{q_{2}} & -\underline{F}_{0} & 0 & -\underline{F}_{q_{0}} & -\underline{F}_{q_{3}} & \underline{E}_{1} & \underline{H}_{1}-\underline{H}
\end{array}\right)
$$

which preserve the $\mathrm{SO}(4,4)$ quadratic form is $\eta=\left(\begin{array}{cc}0 & 1_{4} \\ 1_{4} & 0\end{array}\right), X^{t} \eta+\eta X=0$. This basis is adapted to the branching

$$
\mathfrak{s o}(4,4)=[\mathfrak{s l}(2, \mathbb{R})]^{4} \oplus(2,2,2,2),
$$

where the four commuting $\mathrm{SL}(2, \mathbb{R})$ subgroups are generated by

$$
\left[E_{i}, F_{i}\right]=H_{i}, \quad\left[H_{i}, E_{i}\right]=2 E_{i}, \quad\left[H_{i}, F_{i}\right]=-2 F_{i} \quad i=0,1,2,3
$$

while the 16 real generators in the coset fit in an hypercube $E_{a_{0}, a_{1}, a_{2}, a_{3}}$ with $a_{i}=1,2$ (see figure 4). Decomposing with respect to the Cartan generator $H_{0}$ leads to the real 5 -grading

$$
\left.\left.\left.\left.\left.F_{0}\right|_{-2} \oplus\left\{F_{p^{\Lambda}}, F_{q_{\Lambda}}\right\}\right|_{-1} \oplus\left(H \oplus\left\{E_{i}, F_{i}, H_{i}\right\}_{i=1,2,3}\right)\right|_{0} \oplus\left\{E_{p^{\Lambda}}, E_{q_{\Lambda}}\right\}\right|_{1} \oplus E_{0}\right|_{2} .
$$

A canonical basis of Cartan generators is $\left(H_{1}, H_{2}, H_{3} ; \frac{1}{2}\left(H-H_{1}-H_{2}-H_{3}\right)\right)$, where the last generator is the one attached to the middle node of the Dynkin diagram. 


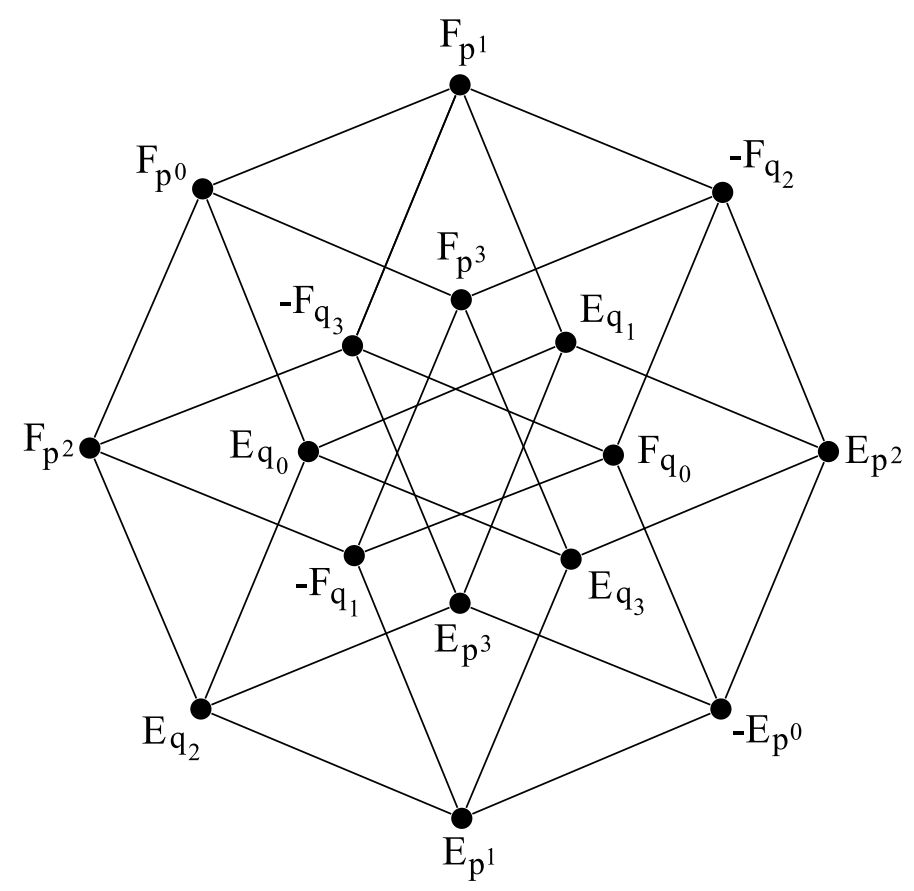

Figure 4. $(2,2,2,2)$ roots in $S O_{0}(4,4)$

The symmetric space $\mathcal{M}_{3}$ can then be parametrized in the Iwasawa gauge by the coset element

$$
\mathcal{V}=e^{-\frac{1}{2} \phi H_{0}} \cdot\left(\prod_{i=1,2,3} e^{-\frac{1}{2}\left(\log S_{2}^{(i)}\right) H_{i}} \cdot e^{-S_{1}^{(i)} E_{i}}\right) \cdot e^{-\zeta^{\Lambda} E_{q_{\Lambda}}-\tilde{\zeta}_{\Lambda} E_{p^{\Lambda}}} \cdot e^{-\frac{1}{2} \sigma E_{0}}
$$

The metric (4.3) on $\mathcal{M}_{3}$ is then the right-invariant metric obtained from the Maurer-Cartan one-form $\theta=d \mathcal{V} \cdot \mathcal{V}^{-1}$,

$$
d s_{\mathcal{M}_{3}}^{2}=\operatorname{Tr}\left(P^{2}\right), \quad P=\frac{1}{2}\left(\theta+\theta^{T}\right),
$$

The Iwasawa parametrization (4.10) can also be used for the pseudo-Riemannian space $\mathcal{M}_{3}^{*}$, although it is suitable only on an open subset of the full $\mathcal{M}_{3}$. The metric (4.4) is obtained as in (4.11) upon replacing $P$ by

$$
P_{*}=\frac{1}{2}\left(\theta+\eta^{\prime} \theta^{T} \eta^{\prime-1}\right), \quad \eta^{\prime}=\operatorname{diag}(-1,-1,1,1,-1,-1,1,1) .
$$

where $\eta^{\prime}$ is the quadratic form preserved by $\mathrm{SL}(2, \mathbb{R})^{4}=\mathrm{SO}(2,2) \times \mathrm{SO}(2,2) \subset G_{3}$.

The Hamiltonian associated to geodesic motion on $\mathcal{M}_{3}^{*}$ with Lagrangian $\mathcal{L}=d s_{\mathcal{M}_{3}^{*}}^{2} / d \rho$ is given by

$$
\begin{aligned}
H= & \frac{1}{4}\left[p_{\phi}^{2}+\sum_{i=1,2,3}\left(S_{2}^{(i)}\right)^{2}\left(p_{S_{1}^{(i)}}^{2}+p_{S_{2}^{(i)}}^{2}\right)\right]+4 e^{2 \phi} p_{\sigma}^{2} \\
& +e^{\phi}\left[\left(P_{\zeta^{\Lambda}}-\operatorname{Re} \mathcal{N}_{\Lambda \Sigma} P_{\tilde{\zeta}_{\Sigma}}\right)[\operatorname{Im} \mathcal{N}]^{\Lambda \Lambda^{\prime}}\left(P_{\zeta^{\Lambda^{\prime}}}-\operatorname{Re} \mathcal{N}_{\Lambda^{\prime} \Sigma^{\prime}} P_{\tilde{\zeta}_{\Sigma^{\prime}}}\right)+P_{\tilde{\zeta}_{\Lambda}}[\operatorname{Im} \mathcal{N}]_{\Lambda \Lambda^{\prime}} P_{\tilde{\zeta}_{\Lambda^{\prime}}}\right],
\end{aligned}
$$


where

$$
P_{\zeta^{\Lambda}}=p_{\zeta^{\Lambda}}-\tilde{\zeta}_{\Lambda} p_{\sigma}, \quad P_{\tilde{\zeta}_{\Lambda}}=p_{\tilde{\zeta}_{\Lambda}}+\zeta^{\Lambda} p_{\sigma}
$$

and $p_{a}=\partial \mathcal{L} / \partial \dot{\phi}^{a}$ are the momenta conjugate to $\phi_{a}$. The conserved Noether charges are given by

$$
Q=\mathcal{V}^{-1} P_{*} \mathcal{V}
$$

The electric and magnetic charges $q_{\Lambda}, p^{\Lambda}$, NUT charge $k$ are proportional to the components $E_{q_{\Lambda}}, E_{p^{\Lambda}}, E_{k}$ of the Noether charge:

$$
\left\{\begin{array}{l}
p^{\Lambda}=\sqrt{2} E_{p^{\Lambda}}=-\frac{\sqrt{2}}{2}\left(p_{\tilde{\zeta}_{\Lambda}}-\zeta^{\Lambda} p_{\sigma}\right) \\
q_{\Lambda}=\sqrt{2} E_{q_{\Lambda}}=-\frac{\sqrt{2}}{2}\left(p_{\zeta^{\Lambda}}+\tilde{\zeta}_{\Lambda} p_{\sigma}\right)
\end{array}, \quad k=2 E_{0}=-2 p_{\sigma} .\right.
$$

Note that the charges $\left(p^{\Lambda}, q_{\Lambda}\right)$ transform as $(2,2,2)$ of $G_{4}$, so can be fit into a cube $E_{2, a_{1}, a_{2}, a_{3}}$, corresponding to the front face of the hypercube in figure 4 . The Cayley hyperdeterminant of this cube,

$$
\begin{aligned}
\diamond= & \frac{1}{8} \epsilon^{a_{1} b_{1}} \epsilon^{a_{2} b_{2}} \epsilon^{a_{3} d_{3}} \epsilon^{c_{1} d_{1}} \epsilon^{c_{2} d_{2}} \epsilon^{b_{3} c_{3}} E_{2, a_{1}, a_{2}, a_{3}} E_{2, b_{1}, b_{2}, b_{3}} E_{2, c_{1}, c_{2}, c_{3}} E_{2, d_{1}, d_{2}, d_{3}} \\
= & -4 q_{0} p^{1} p^{2} p^{3}+4 p^{0} q_{1} q_{2} q_{3}+4\left(p^{1} p^{2} q_{1} q_{2}+p^{2} p^{3} q_{2} q_{3}+p^{3} p^{1} q_{3} q_{1}\right) \\
& -\left(p^{0} q_{0}+p^{1} q_{1}+p^{2} q_{2}+p^{3} q_{3}\right)^{2}
\end{aligned}
$$

provides a quartic polynomial invariant under $[\operatorname{SL}(2, \mathbb{R})]^{3}$. This is in fact the truncation of the $E_{7(7)}$ quartic invariant (2.14) in $\mathcal{N}=8$ supergravity.

For static black holes with $p_{\sigma}=0$, the Hamiltonian (4.13) reduces to

$$
H=\dot{\phi}^{2}+\sum_{i=1,2,3} \frac{\left(\dot{S}_{1}^{(i)}\right)^{2}+\left(\dot{S}_{2}^{(i)}\right)^{2}}{\left(S_{2}^{(i)}\right)^{2}}-e^{\phi} V_{\mathrm{BH}},
$$

where $V_{\mathrm{BH}}$ is the "black hole potential"

$$
V_{\mathrm{BH}}=-\frac{1}{2}\left[\left(q_{\Lambda}-\operatorname{Re} \mathcal{N}_{\Lambda \Sigma} p^{\Sigma}\right)[\operatorname{Im} \mathcal{N}]^{\Lambda \Lambda^{\prime}}\left(q_{\Lambda^{\prime}}-\operatorname{Re}_{\Lambda_{\Lambda^{\prime} \Sigma^{\prime}}} p^{\Sigma^{\prime}}\right)+p^{\Lambda}[\operatorname{Im} \mathcal{N}]_{\Lambda \Lambda^{\prime}} p^{\Lambda^{\prime}}\right] .
$$

Note that $V_{\mathrm{BH}}>0$, but the actual potential $-e^{\phi} V_{\mathrm{BH}}$ is unbounded from below.

Since $\mathcal{M}_{3}^{*}$ has restricted holonomy group $\mathrm{SO}(2,2)^{2}$, it is possible to construct a vielbein $V_{A A^{\prime} A^{\prime \prime} A^{\prime \prime \prime}}$ transforming as $(2,2,2,2)$, covariantly constant under the $\mathfrak{s l}(2, \mathbb{R})^{4}$-valued spin connection. In order to make contact with the standard construction of the quaternionic vielbein for the $c$-map geometry (4.3) [31], we adapt the construction of [37] to the pseudoRiemannian case.

For this purpose, we perform a Cayley rotation within each $\mathrm{SL}(2)$ factor, and define

$$
J_{i}^{+}=\frac{1}{2}\left(E_{i}+F_{i}+i H_{i}\right), \quad J_{i}^{3}=E_{i}-F_{i}, \quad J_{i}^{-}=\frac{1}{2}\left(E_{i}+F_{i}-i H_{i}\right),
$$

for $i=0,1,2,3$. This defines a $[\mathrm{SL}(2, \mathbb{C})]^{4}$ subgroup of $\mathrm{SO}(8, \mathbb{C})$. Under the branching

$$
\mathfrak{s o}(4,4) \cong[\mathfrak{s u}(2, \mathbb{R})]^{4} \oplus(2,2,2,2),
$$



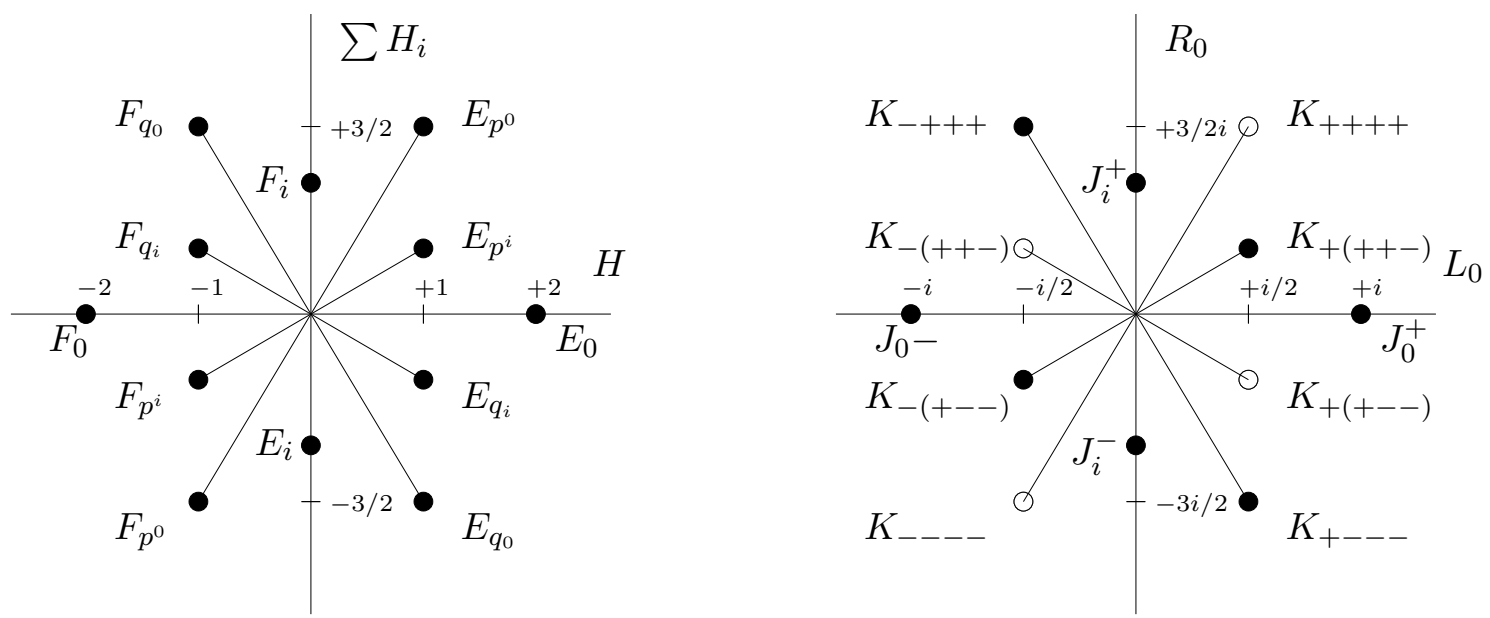

Figure 5. Two-dimensional projection of the root diagram with respect to the split Cartan torus $H_{0}, H_{1}+H_{2}+H_{3}$ (left) and the compact Cartan torus $L_{0}=J_{0}^{3}, R_{0}=J_{1}^{3}+J_{2}^{3}+J_{3}^{3}$ (right). The compact (resp. non-compact) roots are indicated by a white (resp. black) dot. The long roots generate $\mathrm{SL}(3, \mathbb{R})$ (left) and $\mathrm{SU}(2,1)$ (right) subgroups, respectively.

the 16 generators in the pseudo-real coset $(2,2,2,2)$ can now be fit in a complex hypercube $K_{A_{0}, A_{1}, A_{2}, A_{3}}$ with $A_{i}= \pm 1$, where $i A_{i}$ is the eigenvalue under $J_{i}^{3}$ and $K$ satisfies the reality condition

$$
K_{-A_{0},-A_{1},-A_{2},-A_{3}}=\left(K_{A_{0}, A_{1}, A_{2}, A_{3}}\right)^{*} .
$$

The entries in $K$ can be obtained from the real coset $E_{a_{0}, a_{1}, a_{2}, a_{3}}$ by means of a Cayley rotation $C=e^{-\frac{i \pi}{4} \sum_{i=0 \ldots 3}\left(E_{i}+F_{i}\right)}$, for example,

$$
\begin{aligned}
K_{++++}= & C \cdot F_{p^{0}} \cdot C^{-1} \\
= & \frac{1}{4}\left(-E_{p^{0}}-i E_{p^{1}}-i E_{p^{2}}-i E_{p^{3}}+i E_{q_{0}}-E_{q_{1}}-E_{q_{2}}-E_{q_{3}}\right. \\
& \left.+F_{p^{0}}+i F_{p^{1}}+i F_{p^{2}}+i F_{p^{3}}-i F_{q_{0}}+F_{q_{1}}+F_{q_{2}}+F_{q_{3}}\right)
\end{aligned}
$$

The action of $L_{0} \equiv J_{0}^{3}$ defines a complex 5 -grading, and the combined action of $L_{0}$ and $R_{0}=J_{1}^{3}+J_{2}^{3}+J_{3}^{3}$ projects the root system onto the $G_{2}$ root system, with multiplicity 3 for the short roots. We now perform a Weyl reflection with respect to $K_{+---}$, in such a way that the four $\mathrm{SU}(2)$ subalgebras generated by $J_{0}^{ \pm}, J_{i}^{ \pm}$are rotated by an angle $\pi / 3$ into the four $\mathrm{SU}(2)$ subalgebra generated by ${ }^{23} K_{ \pm \pm \pm \pm}$and $K_{ \pm( \pm \mp \mp)}$, with Cartan generators

$$
J_{0}^{3}+J_{1}^{3}+J_{2}^{3}+J_{3}^{3}, \quad J_{0}^{3} \pm J_{1}^{3} \mp J_{2}^{3} \mp J_{3}^{3},
$$

respectively. The algebra $\mathfrak{s o}(4,4)$ again decomposes as $[\mathfrak{s u}(2)]^{4} \oplus(2,2,2,2)$ with respect to this $\mathrm{SU}(2)^{4}$, and the projection of the Maurer-Cartan one-form on the coset yields the desired vielbein. This may be represented in the same figure 4, upon replacing

$$
\begin{aligned}
& E_{p^{0}} \mapsto-\bar{v}, \quad E_{p^{i}} \mapsto i E_{(i)}, \quad E_{q_{i}} \mapsto e_{(i)}, \quad E_{q_{0}} \mapsto-i u, \\
& F_{p^{0}} \mapsto-\bar{v}, \quad F_{p^{i}} \mapsto-i \bar{E}_{(i)}, \quad F_{q_{i}} \mapsto i \bar{e}_{(i)}, \quad F_{q_{0}} \mapsto i \bar{u},
\end{aligned}
$$

\footnotetext{
${ }^{23}$ Here the parenthesis indicates that one should include the three possible permutations.
} 
where $u, v, e^{(i)}, E^{(i)}$ denote the right-invariant one-forms

$$
\begin{array}{rlrl}
v & =-d \phi+\frac{i}{2} e^{-\phi}\left(d \sigma+\tilde{\zeta}^{\Lambda} d \zeta_{\Lambda}-\zeta^{\Lambda} d \tilde{\zeta}_{\Lambda}\right), \quad u & =\sqrt{2} e^{(K-\phi) / 2} X^{\Lambda}\left(d \tilde{\zeta}_{\Lambda}+\mathcal{N}_{\Lambda \Sigma} d \zeta^{\Sigma}\right) \\
e^{(i)} & =i\left(d S_{1}^{(i)}+i d S_{2}^{(i)}\right) / S_{2}^{(i)}, & E^{(i)} & =2 i \sqrt{2} S_{2}^{(i)} e^{-\phi / 2} f_{i}^{\Lambda}\left(d \tilde{\zeta}_{\Lambda}+\overline{\mathcal{N}}_{\Lambda \Sigma} d \zeta^{\Sigma}\right)
\end{array}
$$

where $f_{i}^{I}=e^{K / 2} D_{i} X^{I}=e^{K / 2}\left[\partial_{i}+\left(\partial_{i} K\right)\right] X^{I}$. It is straightforward to check that the vielbein $V_{A A^{\prime} A^{\prime \prime} A^{\prime \prime \prime}}$ satisfies the reality condition

$$
V_{A A^{\prime} A^{\prime \prime} A^{\prime \prime \prime}}=\epsilon_{A B} \epsilon_{A^{\prime} B^{\prime}} \epsilon_{A^{\prime \prime} B^{\prime \prime}} \epsilon_{A^{\prime \prime \prime} B^{\prime \prime \prime}}\left(V_{B B^{\prime} B^{\prime \prime} B^{\prime \prime \prime}}\right)^{*}
$$

In term of these one-forms, the metric takes the simple form

$d s_{\mathcal{M}_{3}}^{2}=-\frac{1}{2} \epsilon_{A B} \epsilon_{A^{\prime} B^{\prime}} \epsilon_{A^{\prime \prime} B^{\prime \prime}} \epsilon_{A^{\prime \prime \prime} B^{\prime \prime \prime}} V_{A A^{\prime} A^{\prime \prime} A^{\prime \prime \prime}} V_{B B^{\prime} B^{\prime \prime} B^{\prime \prime \prime}}=u \bar{u}+v \bar{v}+\sum_{i=1,2,3}\left(e^{(i)} \bar{e}^{(i)}+E^{(i)} \bar{E}^{(i)}\right)$

The spin connection for each of the $\mathrm{SU}(2)$ factors is given by

$$
\left(\begin{array}{c}
\underline{K}_{++++} \\
\underline{J}_{3} \\
\underline{K}_{----}
\end{array}\right)=\left(\begin{array}{c}
u \\
\frac{1}{2}\left((v-\bar{v})+\left(e_{1}-\bar{e}_{1}\right)+\left(e_{2}-\bar{e}_{2}\right)+\left(e_{3}-\bar{e}_{3}\right)\right) \\
-\bar{u}
\end{array}\right)
$$

and

$$
\left(\begin{array}{c}
\underline{K}_{-(++-)} \\
\underline{J}_{i}^{\prime} \\
\underline{K}_{+(+--)}
\end{array}\right)=\left(\begin{array}{c}
E_{i} \\
\frac{1}{2}\left((v-\bar{v})+\left(e_{i}-\bar{e}_{i}\right)-\left(e_{j}-\bar{e}_{j}\right)-\left(e_{k}-\bar{e}_{k}\right)\right) \\
-\bar{E}_{i}
\end{array}\right)
$$

The constant covariance of the vielbein can be checked using the Maurer-Cartan equation $d \theta+\theta \wedge \theta=0$

$$
\begin{aligned}
d u+\frac{1}{4}\left(\left(e_{1}-\bar{e}_{1}\right)+\left(e_{2}-\bar{e}_{2}\right)+\left(e_{3}-\bar{e}_{3}\right)-(v+\bar{v})\right) u & =\frac{1}{2} e_{i} \wedge E_{i} \\
d E_{i}+\frac{1}{4}\left(\left(e_{j}-\bar{e}_{j}\right)+\left(e_{k}-\bar{e}_{k}\right)-\left(e_{i}-\bar{e}_{i}\right)-(v+\bar{v})\right) E_{i} & =\frac{1}{2}\left(\left|\epsilon_{i j k}\right| \bar{E}_{j} \wedge e_{k}+u \wedge \bar{e}_{i}\right) \\
d v & =\frac{1}{2}\left(v \bar{v}+u \bar{u}+E_{i} \bar{E}_{i}\right) \\
d e_{i}+\frac{1}{2} \bar{e}_{i} e_{i} & =0
\end{aligned}
$$

Singling out the first SU(2) as the R-symmetry group, we recover the quaternionic vielbein for the $c$-map geometry (4.3) [31],

$$
V^{\alpha A}=\left(\begin{array}{cc}
\bar{u} & v \\
\bar{e}^{i} & E_{i} \\
-\bar{E}_{\bar{i}} & e^{i} \\
-\bar{v} & u
\end{array}\right)=-i\left(\begin{array}{cc}
\underline{K}_{+---} & \underline{J}_{0}^{+} \\
-\underline{J}_{i}^{-} & \underline{K}_{+(++-)} \\
\underline{K}_{-(+--)} & \underline{J}_{i}^{+} \\
\underline{J}_{0}^{-} & -\underline{K}_{-+++}
\end{array}\right),
$$

Finally, in order to describe the pseudo-Riemannian manifold $\mathcal{M}_{3}^{*}$ we conjugate the generators by $\exp \left(i \pi H_{0} / 2\right)$, whose effect is to Wick rotate

$$
E_{0} \mapsto-E_{0}, \quad\left(\begin{array}{c}
E_{p^{\Lambda}} \\
E_{q_{\Lambda}}
\end{array}\right) \mapsto i\left(\begin{array}{c}
E_{p^{\Lambda}} \\
E_{q_{\Lambda}}
\end{array}\right), \quad\left(\begin{array}{c}
F_{p^{\Lambda}} \\
F_{q_{\Lambda}}
\end{array}\right) \mapsto-i\left(\begin{array}{c}
F_{p^{\Lambda}} \\
F_{q_{\Lambda}}
\end{array}\right), \quad F_{0} \mapsto-F_{0} .
$$


The $(2,2,2,2)$ coset $V_{A A^{\prime} A^{\prime \prime} A^{\prime \prime \prime}}$ is now obtained from figure 4 by replacing

$$
\begin{aligned}
E_{p^{0}} \mapsto-\bar{v}, \quad E_{p^{i}} \mapsto E_{(i)}, \quad E_{q_{i}} \mapsto e_{(i)}, \quad E_{q_{0}} \mapsto-u, \\
F_{p^{0}} \mapsto-v, \quad F_{p^{i}} \mapsto-\bar{E}_{(i)}, \quad F_{q_{i}} \mapsto \bar{e}_{(i)}, \quad F_{q_{0}} \mapsto \bar{u},
\end{aligned}
$$

In terms of this new vielbein $V_{A A^{\prime} A^{\prime \prime} A^{\prime \prime \prime}}^{*}$, the pseudo-Riemannian metric (4.4) may be written as

$$
\begin{aligned}
d s_{\mathcal{M}_{3}^{*}}^{2} & =-\frac{1}{2} \epsilon_{A B} \epsilon_{A^{\prime} B^{\prime}} \epsilon_{A^{\prime \prime} B^{\prime \prime}} \epsilon_{A^{\prime \prime \prime} B^{\prime \prime \prime}} V_{A A^{\prime} A^{\prime \prime} A^{\prime \prime \prime}}^{*} V_{B B^{\prime} B^{\prime \prime} B^{\prime \prime \prime}}^{*} \\
& =\left(v \bar{v}+\sum_{i=1,2,3} e^{(i)} \bar{e}^{(i)}\right)-\left(u \bar{u}+\sum_{i=1,2,3} E^{(i)} \bar{E}^{(i)}\right) .
\end{aligned}
$$

As will become clear in section 4.3, the two terms in bracket correspond to the kinetic and potential terms in the Hamiltonian (4.18).

\subsection{Near horizon solutions}

For simplicity, we focus on solutions with $D 4$ and $D 0$ brane charges only, and denote $P^{i}=p^{i}, P^{0}=-p^{0}, Q_{i}=q_{i}, Q_{0}=-q_{0}$ to conform with existing literature. For extremal solutions, the near horizon geometry is $A d S_{2} \times S_{2}$ with constant values for the complex scalars $S^{(i)}$, which have to extremize the "black hole potential" $V_{\mathrm{BH}}$. For $D 4-D 0$, it is consistent to set the axions $S_{1}^{(i)}$ to zero. In this case, $V_{\mathrm{BH}}$ simplifies to

$$
V_{\mathrm{BH}}=\frac{Q_{0}^{2}+\left(P^{1}\right)^{2} T_{2}^{2} U_{2}^{2}+\left(P^{2}\right)^{2} S_{2}^{2} U_{2}^{2}+\left(P^{3}\right)^{2} S_{2}^{2} T_{2}^{2}}{2 S_{2} T_{2} U_{2}} .
$$

Extremization with respect to $S_{2}, T_{2}, U_{2}$ leads to a unique minimum,

$$
S_{2, *}=\sqrt{\frac{\left|Q_{0} P^{1}\right|}{\left|P^{2} P^{3}\right|}}, \quad T_{2, *}=\sqrt{\frac{\left|Q_{0} P^{2}\right|}{\left|P^{1} P^{3}\right|}}, \quad U_{2, *}=\sqrt{\frac{\left|Q_{0} P^{3}\right|}{\left|P^{1} P^{2}\right|}},
$$

corresponding to an entropy

$$
S_{\mathrm{BH}}=\pi V_{\mathrm{BH}, *}=2 \pi \sqrt{\left|Q_{0} P^{1} P^{2} P^{3}\right|} .
$$

The extremum (4.39) exists for charges $Q_{0}, P^{i}$ of any sign, but the supersymmetry properties of the solution do depend on the signs of the charges. The values of the central charges at the horizon are summarized in table 1 , where $z=\operatorname{sgn}\left(Q_{0}\right) \sqrt{2}\left|Q_{0} P^{1} P^{2} P^{3}\right|^{1 / 4}$ (see also $[18,29,33]$ ): Case (a) corresponds to BPS black holes of the $S T U$ model. The next three cases $(b c d)$ are the so-called non-BPS, $Z_{*}=0$ extremal solutions. They are non-BPS in the $S T U$ model, but can still be lifted to 1/8-BPS black holes in maximal supergravity [48]. All these cases have $\diamond>0$. In contrast, the remaining four cases $($ efgh $)$ have $\diamond<0$ and are genuinely non-BPS. They are characterized by the fact that the four central charges $Z, Z_{i}$ at the horizon are non-vanishing, and in fact equal in modulus. We shall discuss these solutions in more detail below. 


\begin{tabular}{|c|ccc|cccc|}
\hline & $P^{1} Q_{0}$ & $P^{2} Q_{0}$ & $P^{3} Q_{0}$ & $Z_{*}$ & $Z_{1, *}$ & $Z_{2, *}$ & $Z_{3, *}$ \\
\hline$(a)$ & + & + & + & $-z$ & 0 & 0 & 0 \\
$(b)$ & + & - & - & 0 & $-z$ & 0 & 0 \\
$(c)$ & - & + & - & 0 & 0 & $-z$ & 0 \\
$(d)$ & - & - & + & 0 & 0 & 0 & $-z$ \\
\hline$(e)$ & - & - & - & $\frac{z}{2}$ & $-\frac{z}{2}$ & $-\frac{z}{2}$ & $-\frac{z}{2}$ \\
$(f)$ & - & + & + & $-\frac{z}{2}$ & $\frac{z}{2}$ & $-\frac{z}{2}$ & $-\frac{z}{2}$ \\
$(g)$ & + & - & + & $-\frac{z}{2}$ & $-\frac{z}{2}$ & $\frac{z}{2}$ & $-\frac{z}{2}$ \\
$(h)$ & + & + & - & $-\frac{z}{2}$ & $-\frac{z}{2}$ & $-\frac{z}{2}$ & $\frac{z}{2}$ \\
\hline
\end{tabular}

Table 1. Central charges at the horizon.

\subsection{Extremal $Z_{*}=0$ solutions}

In this subsection, we discuss the geodesic flow interpretation of BPS black holes, as well as so-called non-BPS, $Z_{*}=0$ black holes, as they all appear on the same footing in the context of the $S T U$ model.

Our starting point is the $\operatorname{SL}(2, \mathbb{R})^{4}$ vielbein $V_{A A^{\prime} A^{\prime \prime} A^{\prime \prime \prime}}$ computed at the end of section 4.1 in terms of the invariant one-forms (4.27), and the relation (4.16) between the electromagnetic and NUT charges $q_{\Lambda}, p^{\Lambda}, k$ and canonical momenta conjugate to $\zeta^{\Lambda}, \tilde{\zeta}_{\Lambda}, \sigma$. For a static black hole, $k=-2 p_{\sigma}=0$, which allows to express the differentials $d \zeta^{\Lambda}, d \tilde{\zeta}_{\Lambda}, d \sigma$ in terms of the electromagnetic charges $q_{\Lambda}, p^{\Lambda}$. In this way, we find

$$
\begin{aligned}
v & =-d \phi, & u & =-i e^{\phi / 2} Z \\
e^{(i)} & =i\left(d S_{1}^{(i)}+i d S_{2}^{(i)}\right) / S_{2}^{(i)}, & E^{(i)} & =-i e^{\phi / 2} Z_{i}
\end{aligned}
$$

where $Z \equiv Z_{0}$ and $Z_{i}$ are the central charge and "scalar charges", respectively,

$$
Z=e^{K / 2}\left(q_{\Lambda} X^{\Lambda}-p^{\Lambda} F_{\Lambda}\right), \quad Z_{i}=-2 i S_{2}^{(i)}\left(\partial_{i}+\frac{1}{2} \partial_{i} K\right) Z
$$

For completeness we list the central charges:

$$
\begin{aligned}
2 \sqrt{2 S_{2} T_{2} U_{2}} Z= & \left(q_{0}+q_{1} S_{1}+q_{2} T_{1}+q_{3} U_{1}+p^{3} S_{1} T_{1}+p^{2} S_{1} U_{1}+p^{1} T_{1} U_{1}-p^{0} S_{1} T_{1} U_{1}\right) \\
& +i S_{2}\left(q_{1}+p^{3} T_{1}+\left(p^{2}-p^{0} T_{1}\right) U_{1}\right)+i T_{2}\left(q_{2}+p^{3} S_{1}+\left(p^{1}-p^{0} S_{1}\right) U_{1}\right) \\
& +i U_{2}\left(q_{3}+p^{2} S_{1}+\left(p^{1}-p^{0} S_{1}\right) T_{1}\right)-S_{2} T_{2}\left(p^{3}-p^{0} U_{1}\right) \\
& -S_{2} U_{2}\left(p^{2}-p^{0} T_{1}\right)-T_{2} U_{2}\left(p^{1}-p^{0} S_{1}\right)+i p^{0} S_{2} T_{2} U_{2}, \\
2 \sqrt{2 S_{2} T_{2} U_{2}} Z_{1}= & \left(q_{0}+q_{1} S_{1}+q_{2} T_{1}+q_{3} U_{1}+p^{3} S_{1} T_{1}+p^{2} S_{1} U_{1}+p^{1} T_{1} U_{1}-p^{0} S_{1} T_{1} U_{1}\right) \\
& -i S_{2}\left(q_{1}+p^{3} T_{1}+\left(p^{2}-p^{0} T_{1}\right) U_{1}\right)+i T_{2}\left(q_{2}+p^{3} S_{1}+\left(p^{1}-p^{0} S_{1}\right) U_{1}\right) \\
& +i U_{2}\left(q_{3}+p^{2} S_{1}+\left(p^{1}-p^{0} S_{1}\right) T_{1}\right)+S_{2} T_{2}\left(p^{3}-p^{0} U_{1}\right) \\
& +S_{2} U_{2}\left(p^{2}-p^{0} T_{1}\right)+T_{2} U_{2}\left(-p^{1}+p^{0} S_{1}\right)-i p^{0} S_{2} T_{2} U_{2}, \\
2 \sqrt{2 S_{2} T_{2} U_{2}} Z_{2}= & \left(q_{0}+q_{1} S_{1}+q_{2} T_{1}+q_{3} U_{1}+p^{3} S_{1} T_{1}+p^{2} S_{1} U_{1}+p^{1} T_{1} U_{1}-p^{0} S_{1} T_{1} U_{1}\right) \\
& \left.+i S_{2}\left(q_{1}+p^{3} T_{1}+\left(p^{2}-p^{0} T_{1}\right) U_{1}\right)-i T_{2}\left(q_{2}+p^{3} S_{1}+\left(p^{1}-p^{0} S_{1}\right) U_{1}\right)\right)
\end{aligned}
$$




$$
\begin{aligned}
& +i U_{2}\left(q_{3}+p^{2} S_{1}+\left(p^{1}-p^{0} S_{1}\right) T_{1}\right)+S_{2} T_{2}\left(p^{3}-p^{0} U_{1}\right) \\
& +S_{2} U_{2}\left(-p^{2}+p^{0} T_{1}\right)+T_{2} U_{2}\left(p^{1}-p^{0} S_{1}\right)-i p^{0} S_{2} T_{2} U_{2} \\
2 \sqrt{2 S_{2} T_{2} U_{2}} Z_{3}= & \left(q_{0}+q_{1} S_{1}+q_{2} T_{1}+q_{3} U_{1}+p^{3} S_{1} T_{1}+p^{2} S_{1} U_{1}+p^{1} T_{1} U_{1}-p^{0} S_{1} T_{1} U_{1}\right) \\
& +i S_{2}\left(q_{1}+p^{3} T_{1}+\left(p^{2}-p^{0} T_{1}\right) U_{1}\right)+i T_{2}\left(q_{2}+p^{3} S_{1}+\left(p^{1}-p^{0} S_{1}\right) U_{1}\right) \\
& -i U_{2}\left(q_{3}+p^{2} S_{1}+\left(p^{1}-p^{0} S_{1}\right) T_{1}\right)+S_{2} T_{2}\left(-p^{3}+p^{0} U_{1}\right) \\
& +S_{2} U_{2}\left(p^{2}-p^{0} T_{1}\right)+T_{2} U_{2}\left(p^{1}-p^{0} S_{1}\right)-i p^{0} S_{2} T_{2} U_{2} .
\end{aligned}
$$

It should be noted that $Z_{i}$ is related to $Z$ by reversal of the sign of one of the $S_{2}^{(i)}$, s followed by complex conjugation, e.g.

$$
Z_{1}\left(S, T, U ; p^{\Lambda}, q_{\Lambda}\right)=\overline{Z\left(\bar{S}, T, U ; p^{\Lambda}, q_{\Lambda}\right)} .
$$

Using (4.37), we see that the black hole potential (4.19) can be rewritten as

$$
V_{\mathrm{BH}}=\left|Z_{0}\right|^{2}+\left|Z_{1}\right|^{2}+\left|Z_{2}\right|^{2}+\left|Z_{3}\right|^{2} .
$$

Moreover, from standard formulae in special geometry [55]

$$
D Z=\frac{i}{2 S_{2}^{(i)}} Z_{i} d S^{(i)}, \quad D Z_{i}=\frac{i}{2 S_{2}^{(k)}}\left|\epsilon_{i j k}\right| \bar{Z}_{j} \wedge d S^{(k)}-\frac{i}{2 S_{2}^{(i)}} Z d \bar{S}^{(i)}
$$

or from the Maurer-Cartan equations (4.32), one can check that

$$
V_{\mathrm{BH}}=W^{2}+4\left(g^{S \bar{S}}\left|\partial_{S} W\right|^{2}+g^{T \bar{T}}\left|\partial_{T} W\right|^{2}+g^{U \bar{U}}\left|\partial_{U} W\right|^{2}\right)
$$

for any choice of $W$ amongst [13]

$$
W=|Z|, \quad W=\left|Z_{1}\right|, \quad W=\left|Z_{2}\right|, \quad W=\left|Z_{3}\right| .
$$

Therefore, any $W$ in (4.51) can be used as a fake superpotential to generate first order equations

$$
\dot{\phi}=-e^{\phi / 2} W, \quad \dot{S}^{(i)}=-e^{\phi / 2} g^{S^{(i)} \bar{S}^{(i)}} \partial_{\bar{S}^{(i)}} W
$$

which imply the second order equations from the Hamiltonian (4.13) at zero energy, and moreover guarantee that the corresponding solution is extremal [12].

The first choice in (4.51) corresponds to BPS black holes. Its interpretation as a special kind of geodesic flow on $\mathcal{M}_{3}^{*}$ is well known [21, 35]: indeed, the BPS attractor flow equations (4.52) with $W=|Z|$ can be written in terms of the invariant forms (4.41) as

$$
v=-z u, \quad \bar{e}_{(i)}=z E_{(i)}, \quad \bar{E}_{(i)}=z e_{(i)}, \quad \bar{u}=-z \bar{v}, \quad z=-i \sqrt{\frac{\bar{Z}}{Z}}
$$

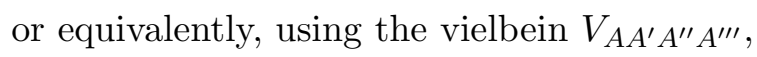

$$
V_{A A^{\prime} A^{\prime \prime} A^{\prime \prime \prime}} \epsilon^{A}=0, \quad \epsilon^{A}=\left(\begin{array}{l}
1 \\
z
\end{array}\right) .
$$

Since the index $A$ transforms as a doublet under the R-symmetry $\mathrm{SU}(2)$, this is recognized as the Killing spinor equation for BPS black holes [21,35]. Note that the phase of $Z$ varies according to

$$
d \arg (Z)+\mathcal{A}=0
$$


where $\mathcal{A}$ is the Kähler connection,

$$
\mathcal{A}=\frac{1}{2 i}\left(\partial_{i} K d t^{i}-\partial_{\bar{i}} K d \overline{\bar{t}}\right)=\frac{1}{2}\left(\frac{d S_{1}}{S_{2}}+\frac{d T_{1}}{T_{2}}+\frac{d U_{1}}{U_{2}}\right) .
$$

Similarly, the first order equations (4.52) with $W=\left|Z_{i}\right|$ can be written in terms of the invariant forms (4.41) as

$$
v=-z \bar{E}_{(i)}, \quad e_{(i)}=z u, \quad e_{(j)}=z E_{(k)}, \quad e_{(k)}=z E_{(j)}, \quad z=-i \sqrt{\frac{Z_{i}}{\bar{Z}_{i}}},
$$

or equivalently, using the vielbein $V_{A A^{\prime} A^{\prime \prime} A^{\prime \prime \prime}}$,

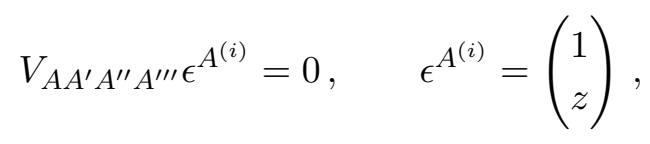

where we denote $A^{(1)}=A^{\prime}, A^{(2)}=A^{\prime \prime}, A^{(3)}=A^{\prime \prime \prime}$. Note that the phase of $Z_{i}$ varies in this class of solutions according to

$$
d \arg \left(Z_{i}\right)+\mathcal{A}_{(i)}=0
$$

where

$$
\mathcal{A}_{(i)}=\frac{1}{2}\left(\frac{d S_{1}^{(i)}}{S_{2}^{(i)}}-\frac{d S_{1}^{(j)}}{S_{2}^{(j)}}-\frac{d S_{1}^{(k)}}{S_{2}^{(k)}}\right) .
$$

Thus, the non-BPS, $Z_{*}=0$ solutions are related to the BPS ones by a permutation of the four SL(2) factors. ${ }^{24}$ This is in accordance with the fact that these solutions lift to $1 / 8$-BPS solutions in maximal supergravity [48]. Using the explicit form of the solutions given in appendix C, one may check that the Noether charge is nilpotent of degree 3 in the vector representation, and corresponds to the nilpotent orbits $\left[(+-+)^{2}(-)^{2}\right]_{I I},\left[(-+-)^{2}(+)^{2}\right]_{I, I I}$ in cases $(\mathrm{a}, \mathrm{b}, \mathrm{c}, \mathrm{d})$, respectively.

\subsection{Extremal $Z_{*} \neq 0$ solutions}

When $\diamond<0$, the extremal black hole is genuinely non-BPS. The fake superpotential $W$ is given by the same formula (2.78) as in $\mathcal{N}=8$ supergravity, upon substituting

$$
\rho_{0}=|Z|, \quad \rho_{1}=\left|Z_{1}\right|, \quad \rho_{2}=\left|Z_{2}\right|, \quad \rho_{3}=\left|Z_{3}\right|, \quad 4 \varphi=\arg \left(Z \bar{Z}_{1} \bar{Z}_{2} \bar{Z}_{3}\right) .
$$

On the sublocus where $\varphi=\pi / 4$ or $\varphi=0$, the simpler forms (B.17), (2.88) can be used. In particular, for axion-free solutions with $Z_{*}<0$ and $Z_{i, *}>0$, both (B.17) and (2.88) reduce to the formula proposed in $[12,18]$

$$
\begin{aligned}
W & =\frac{1}{2}\left(-Z+Z_{1}+Z_{2}+Z_{3}\right) \\
& =e^{K / 2}\left(-Q_{0}+\frac{T \bar{U}+U \bar{T}}{2} P^{1}+\frac{S \bar{U}+U \bar{S}}{2} P^{2}+\frac{S \bar{T}+T \bar{S}}{2} P^{3}\right)
\end{aligned}
$$

on the locus $S_{1}=T_{1}=U_{1}=0$.

Evaluating the Noether charge on the explicit solution given in appendix C, it is easy to confirm that such non-BPS, $Z_{*} \neq 0$ solutions are indeed associated to the nilpotent orbit $[(+-+),(-+-),+,-]$ of $\mathrm{SO}(4,4)$.

\footnotetext{
${ }^{24}$ This fact has been observed independently in [29].
} 


\section{Acknowledgments}

The authors thank G. Dall'Agata, H. Nicolai, K. S. Stelle and S. Trivedi for useful discussions. B.P. acknowledges ICTS, TIFR and the Monsoon Workshop in String Theory 2008 for providing a stimulating atmosphere when this project was begun.

\section{A Nilpotent orbits}

In this appendix, we review some general facts about nilpotent orbits of real Lie groups, and discuss in details the nilpotent orbits of $E_{8(8)}$ and $\mathrm{SO}(4,4)$ relevant for extremal black holes in maximal supergravity and the $S T U$ model, respectively. Useful references for the material of this section are [40] and $[41,44,56]$.

\section{A.1 Generalities}

Complex nilpotent orbits of $G_{\mathbb{C}}$ are classified by conjugacy classes of homomorphisms $\mathfrak{s l}(2) \hookrightarrow \mathfrak{g}_{\mathbb{C}}$, i.e. by triplets $(\mathbf{e}, \mathbf{f}, \mathbf{h})$ of elements in the Lie algebra $\mathfrak{g}_{\mathbb{C}}$ satisfying the $\mathfrak{s l}_{2}$ commutation relations $[\mathbf{e}, \mathbf{f}]=\mathbf{h},[\mathbf{h}, \mathbf{e}]=2 \mathbf{e},[\mathbf{h}, \mathbf{f}]=-2 \mathbf{f}$. Under the adjoint action of $\mathbf{h}$, $\mathfrak{g}_{\mathbb{C}}$ decomposes as a sum of eigenspaces $\mathfrak{g}_{\mathbb{C}}=\bigoplus \mathfrak{g}^{(i)}$ with eigenvalues $i \in[-n, n], n \geq 2$, often referred to as a $2 n+1$-grading (this grading is said to be even if $\mathfrak{g}^{(i)}=0$ for $i$ odd; in this case $n$ is even, and $\mathfrak{g}_{\mathbb{C}}=\bigoplus \mathfrak{g}^{(2 i)}$ yields a $n+1$-grading). Correspondingly, $\mathfrak{g}_{\mathbb{C}}$ decomposes into a sum of irreducible representations of $\mathrm{SL}(2)$ with spin $j \leq n / 2$. e is an element of $\mathfrak{g}^{(2)}$, and therefore nilpotent of degree $2 n+1$. Its complex adjoint orbit $\mathcal{O}_{\mathbb{C}}(\mathbf{e})$ is $P_{\mathbb{C}} \backslash G_{\mathbb{C}}$, where $P_{\mathbb{C}}$ is the stabilizer of $\mathbf{e}$, a non-reductive subgroup of the parabolic subgroup $\bigoplus_{i \geq 0} \mathfrak{g}^{(i)} \subset \mathfrak{g}_{\mathbb{C}}$ which contains in particular $\mathfrak{g}^{(n-1)} \oplus \mathfrak{g}^{(n)}$. The complex dimension of $\mathcal{O}_{\mathbb{C}}(\mathbf{e})$ is equal to $\operatorname{dim} \mathfrak{g}-\operatorname{dim} \mathfrak{g}^{(0)}-\operatorname{dim} \mathfrak{g}^{(1)}$. Complex orbits can be labelled by their Dynkin indices, which are the coefficients of $\mathbf{h}$ on the standard generators of the Cartan subalgebra of $\mathfrak{g}$. These can take values amongst $0,1,2$ only. The subalgebra $\mathfrak{g}^{(0)} \subset \mathfrak{g}_{\mathbb{R}}$ commuting with $\mathbf{h}$ is the direct sum of abelian factors associated to the nodes with a non-zero label, and of the semi-simple Lie algebra whose Dynkin diagram coincides with the set of zero nodes. The set of nilpotent orbits admits a partial ordering, with $\mathbf{e} \leq \mathbf{e}^{\prime}$ whenever $\mathbf{e}^{\prime}$ lies in the closure of the orbit through e. It is convenient to display them in a Hasse-type diagram, with arrows pointing from $\mathbf{e}$ to $\mathbf{e}^{\prime}$ when $\mathbf{e} \leq \mathbf{e}^{\prime}$.

We are interested in nilpotent orbits of $\mathfrak{g}_{\mathbb{R}}$, where $\mathfrak{g}_{\mathbb{R}}$ is the Lie algebra of a noncompact real form $G_{\mathbb{R}}$ of $G_{\mathbb{C}}$, with maximal compact subgroup $K_{\mathbb{R}}$. The maximal compact Lie algebra $\mathfrak{k}_{\mathbb{R}}$ of $\mathfrak{g}_{\mathbb{R}}$ is obtained as the $(-1)$-eigenspace of the Cartan involution ${ }^{\dagger}$ (not to be confused with the adjoint), so that elements of $\mathfrak{k}_{\mathbb{R}}$ are of the form $e-e^{\dagger}$ where $e \in \mathfrak{g}_{\mathbb{R}}$.

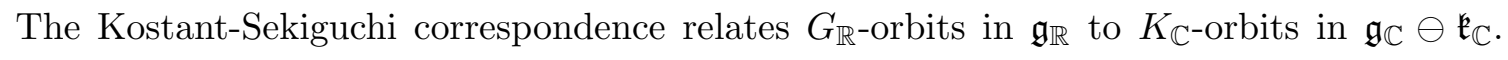
More precisely, the Kostant-Sekiguchi homeomorphism

$$
\mathbf{e} \mapsto \frac{1}{2}\left(\mathbf{e}+\mathbf{e}^{\dagger}+i\left[\mathbf{e}, \mathbf{e}^{\dagger}\right]\right)
$$

identifies

$$
\frac{\mathfrak{N} \cap \mathfrak{g}_{\mathbb{R}}}{G_{\mathbb{R}}} \cong \frac{\mathfrak{N} \cap\left(\mathfrak{g}_{\mathbb{C}} \ominus \mathfrak{k}_{\mathbb{C}}\right)}{K_{\mathbb{C}}}
$$


where $\mathfrak{N}$ is the variety of nilpotent elements inside $\mathfrak{g}_{\mathbb{C}}$. Its inverse can be obtained by use of the complex conjugation *, under the convention that the generators of $\mathfrak{g}_{\mathbb{R}}$ are real,

$$
\mathbf{e} \mapsto \frac{1}{2}\left(\mathbf{e}+\mathbf{e}^{*}-i\left[\mathbf{e}, \mathbf{e}^{*}\right]\right)
$$

The representative of a real orbit $\mathcal{O}_{\mathbb{R}}(\mathbf{e})$ can be chosen so that its standard triplet $(\mathbf{e}, \mathbf{f}, \mathbf{h})$ is a Cayley triplet, i.e. verifies

$$
\mathbf{e}-\mathbf{f} \in \mathfrak{k}_{\mathbb{R}}, \quad \mathbf{h}, \mathbf{e}+\mathbf{f} \in \mathfrak{g}_{\mathbb{R}} \ominus \mathfrak{k}_{\mathbb{R}}
$$

In practice, $\mathbf{f}=\mathbf{e}^{\dagger}$ is obtained from $\mathbf{e}$ via the Cartan involution, after having normalized e properly. The Kostant-Sekiguchi homeomorphism maps this $\mathfrak{s l}_{2}$ subalgebra of $\mathfrak{g}_{\mathbb{R}}$ to the following $\mathfrak{s l}_{2}$ subalgebra of $\mathfrak{g}_{\mathbb{C}}$,

$$
\{\mathbf{e}, \mathbf{f}, \mathbf{h}\} \mapsto\left\{\frac{1}{2}(\mathbf{e}+\mathbf{f}+i \mathbf{h}), \frac{1}{2}(\mathbf{e}+\mathbf{f}-i \mathbf{h}), i(\mathbf{e}-\mathbf{f})\right\}
$$

such that the nilpotent element $\frac{1}{2}(\mathbf{e}+\mathbf{f}+i \mathbf{h})$ lies in $\mathfrak{g}_{\mathbb{C}} \ominus \mathfrak{k}_{\mathbb{C}}$, while the semi-simple element $i(\mathbf{e}-\mathbf{f})$ lies in $\mathfrak{k}_{\mathbb{C}}$.

It turns out that the $G_{\mathbb{R}^{-}}$orbit of $\mathbf{e}$ is uniquely characterized by the $K_{\mathbb{C} \text {-conjugacy }}$ class of $i\left(\mathbf{e}-\mathbf{e}^{\dagger}\right)$. Moreover, one can always choose a representative $\mathbf{e}_{c}$ of the orbit $\mathcal{O}_{\mathbb{R}}(\mathbf{e})$ such that $i\left(\mathbf{e}_{c}-\mathbf{e}_{c}^{\dagger}\right)$ is in a fixed Cartan subalgebra of $\mathfrak{k}_{\mathbb{C}}$. The weighed Dynkin diagram associated to $\mathcal{O}_{\mathbb{R}}(\mathbf{e})$ is then the Dynkin diagram of $K$ decorated by the eigenvalues of the simple roots of $\mathfrak{k}$ with respect to the element $i\left(\mathbf{e}_{c}-\mathbf{e}_{c}^{\dagger}\right)$. This diagram labels the real nilpotent orbit uniquely if $\mathfrak{k}$ is semi-simple, otherwise it has to be supplemented with extra labels associated to the Abelian factors. In contrast to $G$, the labels of the weighed Dynkin diagram of $K$ are not bounded by 2, although they are still positive.

For applications to black holes, we are however more interested in the orbit of an element e lying in $\mathfrak{N} \cap\left(\mathfrak{g}_{\mathbb{R}} \ominus \mathfrak{k}_{\mathbb{R}}^{*}\right)$ under $K_{\mathbb{R}}^{*}$, where $K_{\mathbb{R}}^{*}$ is a non-compact real form of $K_{\mathbb{C}}$ defined by the Cartan involution ${ }^{\ddagger}$. When the intersection $\mathcal{O}_{\mathbb{R}}(\mathbf{e}) \cap\left(\mathfrak{g}_{\mathbb{R}} \ominus \mathfrak{k}_{\mathbb{R}}^{*}\right)$ is non-empty, there exists a "starred" Cayley triplet $\left(\mathbf{e}_{c^{*}}, \mathbf{f}_{c^{*}}=\mathbf{e}_{c^{*}}^{\ddagger}, \mathbf{h}_{c^{*}}\right)$ such that $\mathbf{e}_{c^{*}}, \mathbf{f}_{c^{*}} \in \mathfrak{g}_{\mathbb{R}} \ominus \mathfrak{k}_{\mathbb{R}}^{*}$ and $\mathbf{h}_{c^{*}}$ lies in a fixed Cartan subalgebra of $\mathfrak{k}_{\mathbb{R}}^{*}$. The orbit is characterized by the eigenvalues of the simple roots with respect to $\mathbf{h}_{c^{*}}$, which furnish a weighted Dynkin diagram for $K^{*}$. The semi-simple generator $\mathbf{h}_{c^{*}}$ defines a graded decomposition of the Lie algebra $\mathfrak{k}^{*}$.

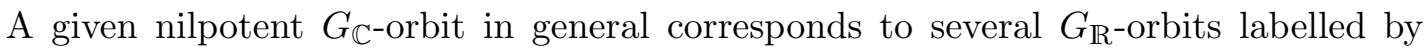
$K_{\mathbb{C}^{-}}$weighted Dynkin diagrams $d_{i}$. Similarly, the $K_{\mathbb{C}^{-}}$orbit associated to $d_{i}$ corresponds to

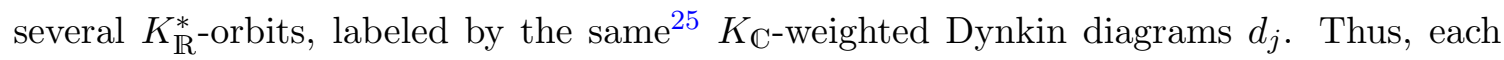
$K_{\mathbb{R}^{*}}^{*}$-orbit is labelled by a pair $\left(d_{i}, d_{j}\right)$. It turns out, although we do not know a proof of this fact, that extremal black holes correspond to choosing $d_{i}=d_{j}$. This condition on $P$ complements the nilpotency condition on $Q$ mentioned in section 1.4.

\footnotetext{
${ }^{25}$ more properly, by a subset thereof. Indeed, some $G_{\mathbb{R}}$-orbits do not intersect with the coset component $\mathfrak{g}_{\mathbb{R}}-\mathfrak{k}^{*}$. This is the case for instance of all the $E_{8(8)}$ nilpotent orbits of dimension strictly greater than 216.
} 


\section{A.2 Nilpotent orbits of $E_{8(8)}$}

\section{Minimal orbit, dimension 58}

We start with the minimal orbit of $E_{8(8)}$, with weighed Dynkin diagram [0000001]. It corresponds to the graded decomposition

$$
\mathfrak{e}_{8(8)} \cong \mathbf{1}^{(-2)} \oplus \mathbf{5 6}^{(-1)} \oplus\left(\mathfrak{g l}_{1} \oplus \mathfrak{e}_{7(7)}\right)^{(0)} \oplus \mathbf{5 6}^{(1)} \oplus \mathbf{1}^{(2)}
$$

of the adjoint representation, familiar from the dimensional reduction from 4 to 3 dimensions. The $\mathbf{3 8 7 5}$ representation is also five-graded with respect to this decomposition, therefore the elements in the grade 2 component are nilpotent of degree 3 in both the adjoint representation and the 3875. To see that such elements can be chosen inside $\mathfrak{e}_{8(8)} \ominus \mathfrak{s o}^{*}(16)$, we decompose (A.6) further into

$$
\mathfrak{e}_{8(8)} \cong \mathbf{1}^{(-2)} \oplus\left(\mathbf{2 8}_{+} \oplus \mathbf{2 8}_{-}\right)^{(-1)} \oplus\left(\mathfrak{g l}_{1} \oplus \mathfrak{s u ^ { * }}(8) \oplus \mathbf{7 0}\right)^{(0)} \oplus\left(\mathbf{2 8}_{+} \oplus \mathbf{2 8}_{-}\right)^{(1)} \oplus \mathbf{1}^{(2)} .
$$

This exhibits the embedding $\mathfrak{s o}^{*}(16) \subset \mathfrak{e}_{8(8)}$, associated to the weighted Dynkin diagram $[.1000000]$ of the real $E_{8(8)}$ orbit

$$
\mathfrak{s o}^{*}(16) \cong \mathbf{2 8}_{+}^{(-1)} \oplus\left(\mathfrak{g l}_{1} \oplus \mathfrak{s u}^{*}(8)\right)^{(0)} \oplus \mathbf{2 8}_{+}^{(1)} .
$$

The weighted Dynkin diagram of the real orbit labels the compact element of the Cartan subalgebra of $\mathfrak{s o}(16)$ that appears in the Cayley triplet associated to the nilpotent element $\mathbf{e}$, namely $\mathbf{e}-\mathbf{e}^{\dagger} \in \mathbf{1}^{(2)}-\mathbf{1}^{(-2)}$. As it turns out, it also labels a non-compact element of the Cartan subalgebra of $\mathfrak{s o}^{*}(16)$ which is $\mathbf{h}=\left[\mathbf{e}, \mathbf{e}^{\ddagger}\right] \in \mathfrak{g l}_{1}^{(0)}$ for a particular choice of normal triplet.

The isotropy subgroups inside $\operatorname{Spin}^{*}(16)$ and $E_{8(8)}$ follow trivially from the fivegraded decomposition

$$
S U^{*}(8) \ltimes \mathbf{2 8}^{(1)} \subset E_{7(7)} \ltimes\left(\mathbf{5 6}^{(1)} \oplus \mathbf{1}^{(2)}\right)
$$

\section{Nilpotent orbit of dimension 92}

The next orbit in the Hasse diagram is associated to the weighted Dynkin diagram [1000000]. The corresponding graded decomposition is the five-graded decomposition of $\mathfrak{e}_{8(8)}$ in term of irreducible representations of $\operatorname{Spin}(7,7)$

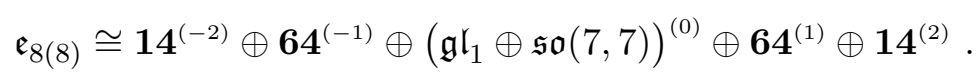

The grade 2 component is no longer a singlet, and one should further decompose $\operatorname{Spin}(7,7)$ irreducible representations with respect to $\operatorname{Spin}(6,7)$ to obtain a singlet in the $\mathbf{1 4} \cong \mathbf{1} \oplus$ 13. ${ }^{26}$ Again it follows from the five-grading that such a nilpotent element satisfy $\mathscr{C}^{3}=0$ in the adjoint representation. The $\mathbf{3 8 7 5}$ is however nine-graded with respect to the $\operatorname{Spin}(7,7)$

\footnotetext{
${ }^{26} \mathrm{~A}$ time-like vector would lead to a minimal orbit. Note that the choice of an either time-like or spacelike vector is equivalent in this case, whereas it would not be in the case of $E_{8(-24)}$, which has a similar five-graded decomposition in irreducible representations of $\operatorname{Spin}(3,11)$. Indeed, there are two corresponding 92 dimensional nilpotent orbits of $E_{8(-24)}$.
} 
decomposition, and only the fifth power of $\mathscr{C}$ vanishes in this representation. One can easily identify the relevant embeddings of $\operatorname{Spin}^{*}(16) / \mathbb{Z}_{2} \subset E_{8(8)}$ such that $\mathbf{1}^{(2)} \subset \mathfrak{e}_{8(8)} \ominus \mathfrak{s o}^{*}(16)$ from the decomposition associated to the weighted Dynkin diagram [..801000] of the orbit,

$$
\mathfrak{s o}^{*}(16) \cong \mathbf{6}^{(-2)} \oplus(\mathbf{4} \otimes \mathbf{8})_{\mathbb{R}}^{(-1)} \oplus\left(\mathfrak{g l}_{1} \oplus \mathfrak{s o}(5,1) \oplus \mathfrak{s o}(2,6)\right)^{(0)} \oplus(\mathbf{4} \otimes \mathbf{8})_{\mathbb{R}}^{(1)} \oplus \mathbf{6}^{(2)}
$$

It turns out that one has two corresponding $\operatorname{Spin}^{*}(16)$ orbits, associated to time-like and space-like vectors $\mathbf{e} \in \mathbf{8}^{(2)}$, which isotropy subgroup of $\mathrm{SO}(2,6)$ are $\mathrm{SO}(1,6)$ and $\mathrm{SO}(2,5)$, respectively. The corresponding isotropy subgroups of $\operatorname{Spin}^{*}(16)$ and the one of $E_{8(8)}$ follow trivially from the five-graded decomposition

$$
\left.\begin{array}{l}
(\operatorname{Spin}(5,1) \times \operatorname{Spin}(1,6)) \ltimes\left((\mathbf{4} \otimes \mathbf{8})_{\mathbb{R}}^{(1)} \oplus \mathbf{6}^{(2)}\right) \\
\operatorname{Spin}(1,5) \times \operatorname{Spin}(5,2)) \ltimes\left((\mathbf{4} \otimes \mathbf{8})_{\mathbb{R}}^{(1)} \oplus \mathbf{6}^{(2)}\right)
\end{array}\right\} \subset \operatorname{Spin}(6,7) \ltimes\left(\mathbf{6 4}^{(1)} \oplus(\mathbf{1} \oplus \mathbf{1 3})^{(2)}\right)
$$

The first line corresponds to regular 1/4-BPS black holes, while the second corresponds to solutions with the two non-saturated central charges higher than the mass i.e. $\left|z_{1}\right|=\left|z_{2}\right|=$ $|W|<\left|z_{3}\right|=\left|z_{4}\right|$.

\section{Nilpotent orbit of dimension 112}

The next nilpotent orbit, with weighted Dynkin diagram [0000010], is slightly more intricate. Its graded decomposition is the seven-graded decomposition in $\mathrm{SL}(2, \mathbb{R}) \times E_{6(6)}$ irreducible representations

$$
\mathfrak{e}_{8(8)} \cong \mathbf{2}^{(-3)} \oplus \overline{\mathbf{2 7}}^{(-2)} \oplus(\mathbf{2} \otimes \mathbf{2 7})^{(-1)} \oplus\left(\mathfrak{g l}_{1} \oplus \mathfrak{s l}_{2} \oplus \mathfrak{e}_{6(6)}\right)^{(0)} \oplus(\mathbf{2} \otimes \overline{\mathbf{2 7}})^{(1)} \oplus \mathbf{2} \mathbf{7}^{(2)} \oplus \mathbf{2}^{(3)} .
$$

To exhibit a singlet in the grade 2 component, one should decompose further into $F_{4(4)}$ irreducible representations,

$$
\begin{array}{r}
\mathfrak{e}_{8(8)} \cong \mathbf{2}^{(-3)} \oplus(\mathbf{1} \oplus \mathbf{2 6})^{(-2)} \oplus(\mathbf{2} \oplus \mathbf{2} \otimes \mathbf{2 6})^{(-1)} \oplus\left(\mathfrak{g l}_{1} \oplus \mathfrak{s l}_{2} \oplus \mathfrak{f}_{4(4)} \oplus \mathbf{2 6}\right)^{(0)} \\
\oplus(\mathbf{2} \oplus \mathbf{2} \otimes \mathbf{2 6})^{(1)} \oplus(\mathbf{1} \oplus \mathbf{2 6})^{(2)} \oplus \mathbf{2}^{(3)}
\end{array}
$$

It follows from the seven-graded decomposition that such a nilpotent element vanishes to the fourth power in the adjoint representation. In order to identify the existence of a corresponding $\operatorname{Spin}^{*}(16)$ orbit inside $\mathfrak{e}_{8(8)} \ominus \mathfrak{s o}^{*}(16)$, one should further decompose ${ }^{27}$

$$
\mathfrak{f}_{4(4)} \cong \mathfrak{s u}(2) \oplus \mathfrak{s p}(3) \oplus\left(\mathbf{2} \otimes \mathbf{1 4}_{3}\right)_{\mathbb{R}}, \quad \mathbf{2 6} \cong(\mathbf{2} \otimes \mathbf{6})_{\mathbb{R}} \oplus \mathbf{1 4} \mathbf{4}_{2} .
$$

The embedding of $\mathfrak{s o}^{*}(16)$ corresponds to the decomposition

$$
\begin{aligned}
\mathfrak{s o}^{*}(16) \cong \mathbf{1}_{+}^{(-3)} & \oplus(\mathbf{2} \otimes \mathbf{6})_{\mathbb{R}}^{(-2)} \oplus\left(\mathbf{1} \oplus(\mathbf{2} \otimes \mathbf{6})_{\mathbb{R}} \oplus \mathbf{1 4}_{2}\right)_{+}^{(-1)} \\
& \oplus\left(\mathfrak{g l}_{1} \oplus \mathfrak{g l} \mathfrak{g l}_{1} \oplus \mathfrak{s u}(2) \oplus \mathfrak{s p}(3) \oplus \mathbf{1 4}_{2}\right)^{(0)} \oplus\left(\mathbf{1} \oplus(\mathbf{2} \otimes \mathbf{6})_{\mathbb{R}} \oplus \mathbf{1 4}_{2}\right)_{-}^{(1)} \\
& \oplus(\mathbf{2} \otimes \mathbf{6})_{\mathbb{R}}^{(2)} \oplus \mathbf{1}_{-}^{(3)}
\end{aligned}
$$

\footnotetext{
${ }^{27}$ We added indices to the $\mathbf{1 4}_{2}$ and the $\mathbf{1 4}_{3}$ representations of $U S p(6)$ to differentiate the real 2-form representation from the pseudo-real 3-form representation.
} 
where the indices \pm denote the eigenvalue \pm under $\mathfrak{g l}_{1} \subset \mathfrak{s l}_{2}$. This decomposition can be read off from the weighted Dynkin diagram [. . to compute the isotropy subgroups of $\operatorname{Spin}^{*}(16)$ and $E_{8(8)}$ as

$$
\begin{aligned}
\left(G L_{+}(1, \mathbb{R}) \times \mathrm{SU}(2) \times\right. & U S p(6)) \ltimes\left(\left((\mathbf{2} \otimes \mathbf{6})_{\mathbb{R}} \oplus \mathbf{1 4}_{2}\right)^{(1)} \oplus(\mathbf{2} \otimes \mathbf{6})_{\mathbb{R}}^{(2)} \oplus \mathbf{1}^{(3)}\right) \\
& \subset\left(\mathrm{SL}(2, \mathbb{R}) \times F_{4(4)}\right) \ltimes\left((\mathbf{2} \otimes \mathbf{2 6})^{(1)} \oplus(\mathbf{1} \oplus \mathbf{2 6})^{(2)} \oplus \mathbf{2}^{(3)}\right)
\end{aligned}
$$

Nevertheless the decomposition (A.16) is not unique, for instance one has a similar decomposition by decomposing $\mathfrak{s u}^{*}(6) \cong \mathfrak{s p}(1,2) \oplus \mathbf{1 4}_{2}$ rather than $\mathfrak{s u}^{*}(6) \cong \mathfrak{s p}(3) \oplus \mathbf{1 4}_{2}$. The resulting orbit has as isotropy subgroup of $\operatorname{Spin}^{*}(16)$

$$
\left(G L_{+}(1, \mathbb{R}) \times \mathrm{SU}(2) \times U S p(2,4)\right) \ltimes\left(\left((\mathbf{2} \otimes \mathbf{6})_{\mathbb{R}} \oplus \mathbf{1 4}_{2}\right)^{(1)} \oplus(\mathbf{2} \otimes \mathbf{6})_{\mathbb{R}}^{(2)} \oplus \mathbf{1}^{(3)}\right)
$$

and correspond to black holes for which some of the central charges are larger than the mass.

\section{Nilpotent orbits of dimension 114}

There are two 114-dimensional orbits which lie in the same complex orbit of $E_{8}$ associated to the weighted Dynkin diagram [ $\left[000_{0002}\right]$. They are associated to the five-graded decomposition (A.6), however the weight 2 indicates that the five grading must be considered as an even nine grading, or equivalently that the corresponding nilpotent elements lie inside the grade 1 component with respect to the five-grading. Because the $\mathbf{3 8 7 5}$ representation decomposes as well into a five-graded decomposition, the representative of these orbit is nilpotent of degree five in both the adjoint and the $\mathbf{3 8 7 5}$ representation. It turns out that the two real orbits are associated to the decomposition of $E_{7(7)}$ under the two real forms $E_{6(2)}$ and $E_{6(6)}$ of $E_{6}$, respectively. In the first case, ${ }^{28}$

$$
\mathfrak{e}_{8(8)} \cong i \mathbb{R}^{(-2)} \oplus(\mathbb{C} \oplus \mathbf{2 7})^{(-1)} \oplus\left(\mathbb{C} \oplus \mathfrak{e}_{6(2)} \oplus \mathbf{2 7}\right)^{(0)} \oplus(\mathbb{C} \oplus \mathbf{2 7})^{(1)} \oplus i \mathbb{R}^{(2)} .
$$

The nilpotent element can be chosen in the real component of $\mathbb{C}^{(1)}$, therefore its nilpotency degree in the adjoint representation is 5 . In order to understand the relevant embedding of $\operatorname{Spin}^{*}(16)$ one should further decompose

$$
\mathfrak{e}_{6(2)} \cong \mathfrak{s u}(2) \oplus \mathfrak{s u}(6) \oplus(\mathbf{2} \otimes \mathbf{2 0})_{\mathbb{R}}, \quad \mathbf{2 7} \cong(\mathbf{2} \otimes \mathbf{6}) \oplus \mathbf{1 5}
$$

and $\mathfrak{s o}^{*}(16)$ into

$$
\mathfrak{s o}^{*}(16) \cong i \mathbb{R}^{(-2)} \oplus(\mathbf{2} \otimes \mathbf{6})^{(-1)} \oplus(\mathbb{C} \oplus \mathfrak{s u}(2) \oplus \mathfrak{s u}(6) \oplus \mathbf{1 5})^{(0)} \oplus(\mathbf{2} \otimes \mathbf{6})^{(1)} \oplus i \mathbb{R}^{(2)},
$$

as suggested by the weighted Dynkin diagram [.080020]. This way one determines the isotropy subgroups inside $\operatorname{Spin}^{*}(16)$ and $E_{8(8)}$ respectively

$$
(\mathrm{SU}(2) \times \mathrm{SU}(6)) \ltimes\left((\mathbf{2} \otimes \mathbf{6})^{(1)} \oplus i \mathbb{R}^{(2)}\right) \subset E_{6(2)} \ltimes\left((\mathbb{R} \oplus \mathbf{2 7})^{(1)} \oplus i \mathbb{R}^{(2)}\right) .
$$

\footnotetext{
${ }^{28}$ Note that the complex representation $\mathbf{2 7}$ is sometimes written as $\mathbf{2 7} \oplus \overline{\mathbf{2 7}}$ in the literature.
} 
It is also interesting to consider the isotropy subgroup inside the $E_{7(7)}$ subgroup of $E_{8(8)}$ which intersects with $\operatorname{Spin}^{*}(16)$ on an SU(8) subgroup. From the embedding

$$
\mathfrak{e}_{7(7)} \cong i \mathbb{R}^{(2)}-i \mathbb{R}^{(-2)} \oplus \mathfrak{e}_{6(2)} \oplus \mathbf{2 7}^{(1)}-\mathbf{2 7}^{(-1)}
$$

one finds that this isotropy subgroup is $E_{6(2)}$. This implies that $\diamond\left(W^{-\frac{1}{2}} Z\right)>0$ on the corresponding $\operatorname{Spin}^{*}(16)$ orbit.

The other nilpotent orbit of dimension 114 is associated to the decomposition

$$
\begin{aligned}
& \mathfrak{e}_{8(8)} \cong \mathbf{1}^{(-2)} \oplus\left(\mathbf{1}^{(-3)} \oplus \mathbf{2} \mathbf{7}^{(-1)} \oplus \overline{\mathbf{2}}^{(1)} \oplus \mathbf{1}^{(3)}\right)^{(-1)} \oplus\left(\mathfrak{g l}_{1} \oplus \overline{\mathbf{2 7}}^{(-2)} \oplus\left(\mathfrak{g l}_{1} \oplus \mathfrak{e}_{6(6)}\right)^{(0)} \oplus \mathbf{2}^{(2)}\right)^{(0)}
\end{aligned}
$$

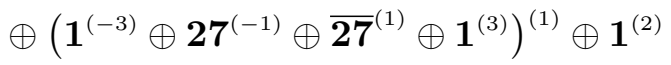

where the internal grading is associated to the three-graded decomposition of $\mathfrak{e}_{7(7)}$ into irreducible representations of $\mathfrak{e}_{6(6)}$. It follows that the nilpotency degree of an element of $\mathbf{1}^{(1,3)}$ in the adjoint representation is 3 . In order to get a nilpotent element of degree 5 in the adjoint representation, one could consider an element in $\mathbf{1}^{(1,-3)}+\mathbf{1}^{(1,3)}$. In order to ensure that this element lies in $\mathfrak{e}_{8(8)} \ominus \mathfrak{s o}^{*}(16)$, we have to further decompose $\mathfrak{e}_{6(6)} \cong \mathfrak{s p}(4) \oplus \mathbf{4 2}$ and consider the embedding of $\mathfrak{s o}^{*}(16)$ associated to its decomposition [. 2800000$]$

$$
\begin{array}{r}
\mathfrak{s o}^{*}(16) \cong\left(\mathbf{1}^{(-3)}-\mathbf{1}^{(3)} \oplus \mathbf{2}^{(-1)}-\mathbf{2 7}^{(1)}\right)^{(-1)} \oplus\left(\mathfrak{g l}_{1} \oplus \mathfrak{s p}(4)^{(0)} \oplus \mathbf{2} \mathbf{7}^{(-2)}+\mathbf{2} \mathbf{7}^{(2)}\right)^{(0)} \\
\oplus\left(\mathbf{1}^{(-3)}-\mathbf{1}^{(3)} \oplus \mathbf{2} \mathbf{7}^{(-1)}-\mathbf{2} \mathbf{7}^{(1)}\right)^{(1)}
\end{array}
$$

These decompositions enable us to compute the corresponding isotropy subgroups inside $\operatorname{Spin}^{*}(16)$ and $E_{8(8)}$,

$$
U S p(8) \ltimes \mathbf{2 7}^{(1)} \subset E_{6(6)} \ltimes\left((\mathbf{1} \oplus \mathbf{2 7} \oplus \overline{\mathbf{2 7}})^{(1)} \oplus \mathbf{1}^{(2)}\right)
$$

The isotropy subgroup inside the $E_{7(7)}$ subgroup of $E_{8(8)}$ which intersects with $\operatorname{Spin}^{*}(16)$ on an $\mathrm{SU}(8)$ subgroup is $E_{6(6)}$, as follows from the decomposition

$$
\mathfrak{e}_{7(7)} \cong \overline{\mathbf{2}}^{(-1,1)}-\overline{\mathbf{2}}^{(1,1)} \oplus \mathbf{1}^{(-2,0)}-2 \mathbf{1}^{(0,0)}+\mathbf{1}^{(2,0)} \oplus \mathfrak{e}_{6(6)} \oplus \mathbf{2}^{(-1,-1)}-\mathbf{2}^{(1,-1)} .
$$

This implies that $\diamond\left(W^{-\frac{1}{2}} Z\right)<0$ on the corresponding $\operatorname{Spin}^{*}(16)$ orbit.

Using similar techniques for different decompositions of $\mathfrak{s o}^{*}(16)$, one gets additional $\operatorname{Spin}^{*}(16)$ orbits with the following isotropy subgroups

$$
\begin{gathered}
\left.\begin{array}{c}
U S p(2,6) \ltimes \mathbf{2 7}^{(1)} \\
(\mathrm{SU}(2) \times \mathrm{SU}(2,4)) \ltimes\left((\mathbf{2} \otimes \mathbf{6})^{(1)} \oplus i \mathbb{R}^{(2)}\right)
\end{array}\right\} \\
\left.\begin{array}{c}
U S p(4,4) \ltimes \mathbf{2 7}^{(1)} \\
\left(\mathrm{SU}(2) \times E_{6(2)}^{*}(6)\right) \ltimes\left((\mathbf{2} \otimes \mathbf{6})^{(1)} \oplus i \mathbb{R}^{(2)}\right)
\end{array}\right\} \subset E_{6(6)} \ltimes\left((\mathbf{1} \oplus \mathbf{2 7} \oplus \overline{\mathbf{2 7}})^{(1)} \oplus \mathbf{1}^{(2)}\right)
\end{gathered}
$$

all of them corresponding to black holes with naked singularities. 


\section{A.3 Nilpotent orbits of $\mathrm{SO}(4,4)$}

For $G_{\mathbb{C}}=\mathrm{SO}(N, \mathbb{C})$, complex orbits can be conveniently labeled by Young tableaux, or partitions of $N$, which summarize the decomposition of the $N$-dimensional vector representation of $G_{\mathbb{C}}$ into finite dimensional representations of $\mathrm{SL}(2, \mathbb{C})$, or equivalently the Jordan normal form of the nilpotent element $\mathbf{e}$ in the vector representation. These Young tableaux must be such that lines with even length (equivalently, representations with half integer spin) occur always in pair. Young tableaux with only rows of even length correspond to two different orbits. The closure ordering $\mathbf{e} \leq \mathbf{e}^{\prime}$ holds whenever, for all $p=1 \ldots N$, the number of boxes in the first $p$ columns of the Young tableau of $\mathbf{e}$ is less than the number of boxes in the first $p$ columns of the Young tableau of $\mathbf{e}^{\prime}$. The nilpotent orbits of $\operatorname{SO}(8, \mathbb{C})$ are summarized in the following Hasse diagram,

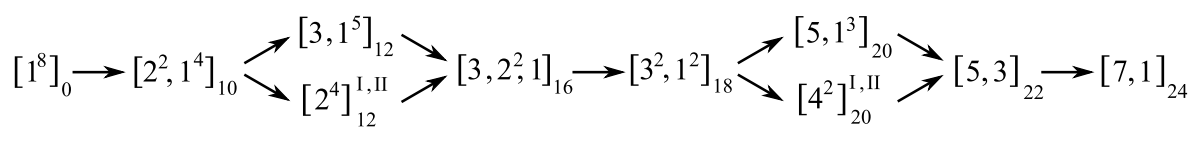

where the integers inside the bracket indicate the size of the Jordan blocks in the 8dimensional vector representation, with multiplicity given in superscript. The subscript indicates the complex dimension of the orbit. The triplets of degenerate orbits $\left[3,1^{5}\right],\left[2^{4}\right]^{I, I I}$ are permuted by triality, as can be easily seen from their Dynkin labels, $\left[20_{0}^{\circ}\right],\left[\begin{array}{c}00_{0}^{2} \\ 0\end{array}\right],\left[00_{2}^{\circ}\right]$. Similarly, $\left[5,1^{3}\right],\left[4^{2}\right]^{I, I I}$, with Dynkin labels $\left[\begin{array}{c}22_{0}^{0} \\ 0\end{array}\right],\left[\begin{array}{ll}0 z_{0}^{2} \\ 0\end{array}\right],\left[\begin{array}{cc}0 z_{2}^{0} \\ 2\end{array}\right]$, are permuted by triality. The remaining orbits, with Dynkin labels $\left[\begin{array}{c}01_{0}^{0} \\ 0\end{array}\right],\left[\begin{array}{c}10_{1}^{1} \\ 1\end{array}\right],\left[\begin{array}{c}02_{0}^{0} \\ 0\end{array}\right],\left[\begin{array}{ll}20_{2}^{2} \\ 2\end{array}\right],\left[\begin{array}{ll}22_{2}^{2} \\ 2\end{array}\right]$ are manifestly triality invariant.

The nilpotent orbits $\mathcal{O}_{\mathbb{R}}(\mathbf{e})$ under the real $\operatorname{group}^{29} G=S O_{0}(p, q, \mathbb{R})$ can be obtained by attaching \pm signs to each of the box of the Young tableau, alternating along each row, such that the total number of $(+,-)$ signs is $(p, q)$ and moreover, such that every row of even length starts with + . A given signed Young tableau corresponds to 4 different orbits labelled $(I, I),(I, I I),(I I, I),(I I, I I)$ when all rows have even length, 2 different orbits labelled $I, I I$ when all rows with odd length have an even number of + signs, 2 different orbits labelled $I, I I$ when all rows with odd length have an even number of - signs, or else a single orbit. All orbits with the same signed Young tableau are related by $O(p, q, \mathbb{R})$. The closure ordering involves not only the shape of the Young tableaux but also the sign and latin letter assignments, and is discussed in detail in [56]. For $G=S O_{0}(4,4)$, the nilpotent orbits of real dimension 18 and lower are summarized in figure 6 .

Starting from the bottom, the smallest (non-zero) orbit is the minimal orbit, or real dimension 10, attached to the Young tableau $\left[(+-)^{2}\right]$ (we omit the rows of length one, for brevity). It is associated to the 5 -grading

$$
\mathfrak{s o}(4,4) \cong \mathbf{1}^{(-2)} \oplus(\mathbf{2} \otimes \mathbf{4})^{(-1)} \oplus\left(\mathfrak{g l}_{1} \oplus \mathfrak{s l}_{2} \oplus \mathfrak{s o}(2,2)\right)^{(0)} \oplus(\mathbf{2} \otimes \mathbf{4})^{(1)} \oplus \mathbf{1}^{(2)}
$$

\footnotetext{
${ }^{29}$ The subscript 0 denotes the component connected to the identity. Recall that $O(p, q, \mathbb{R})$ has four connected components when $p q>0$. The connected component of the identity $S O_{0}(p, q, \mathbb{R})$ is homotopy equivalent to $\mathrm{SO}(p, \mathbb{R}) \times \mathrm{SO}(q, \mathbb{R})$.
} 


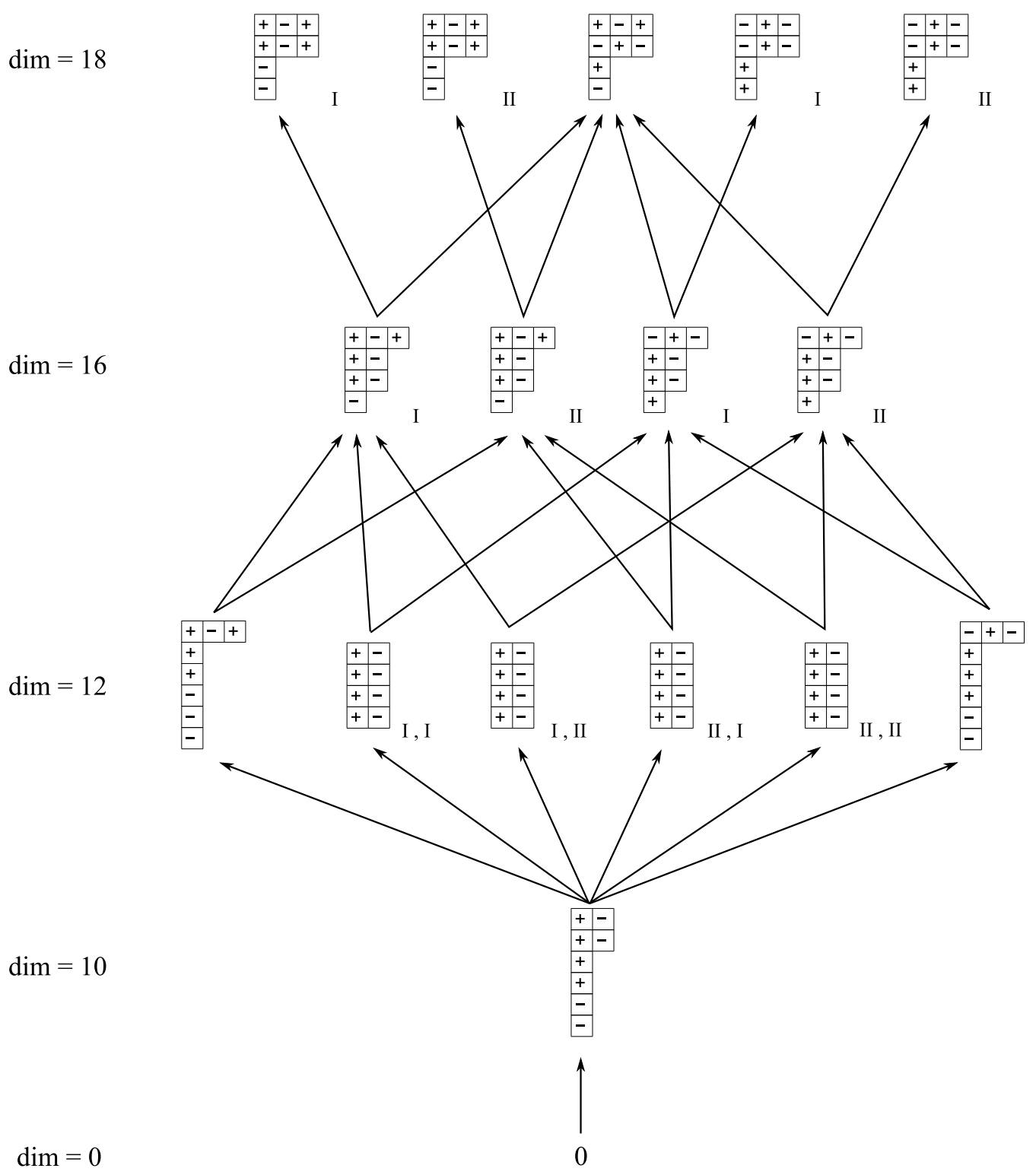

Figure 6. Hasse diagram of nilpotent orbits of $S O_{0}(4,4)$

where $\mathfrak{g}^{(2)}=\mathbb{R} \mathbf{e}, \mathfrak{g}^{(-2)}=\mathbb{R} \mathbf{f}$ and $\mathbf{h}$ is the singlet in $\mathfrak{g}^{(0)}$. This 5-grading originates from the branching $\mathrm{SO}(4,4) \supset \mathrm{SO}(2,2) \times \mathrm{SO}(2,2) \sim \mathrm{SL}(2) \times \mathrm{SL}(2) \times \mathrm{SO}(2,2)$, where $\mathbf{h}$ is the noncompact Cartan generator of the first factor. The stabilizer of $\mathbf{e}$ is $\mathfrak{s o}(2,2, \mathbb{R})^{(0)} \oplus \mathfrak{g}^{(1)} \oplus \mathfrak{g}^{(2)}$, 
therefore the real minimal orbit is isomorphic to

$$
\mathcal{O}_{\min }=\frac{S O_{0}(4,4)}{(\mathrm{SL}(2, \mathbb{R}) \times \mathrm{SO}(2,2, \mathbb{R})) \ltimes\left[(\mathbf{2} \otimes \mathbf{4})^{(1)} \oplus \mathbf{1}^{(2)}\right]}
$$

Since the Young tableau $\left[(+-)^{2}(+)^{2}(-)^{2}\right]$ corresponds to a nilpotent element of order 2 in the vector representation, and is invariant under triality, the minimal orbit is characterized by the nilpotency conditions $V^{2}=S^{2}=C^{2}=A^{3}=0$, where $V, S, C, A$ are a shorthand notation for the representations of $\mathbf{e}$ in the vector, spinor, conjugate spinor and adjoint representations.

The next set of nilpotent orbits, of real dimension 12, corresponds to three distinct unsigned Young tableaux, $\left[3,1^{5}\right]$ and $\left[3^{4}\right]$. These are usually of distinct dimension $2 N-4$ and $4 N-20$, but the dimensions and stabilizers happen to coincide when $N=8$. The tableaux $[( \pm \mp \pm)]$ correspond to the 3 -grading (or more accurately, "even" 5-grading)

$$
\mathfrak{s o}(4,4) \cong \mathbf{6}^{(-2)} \oplus\left(\mathfrak{g l}_{1} \oplus \mathfrak{s o}(3,3)\right)^{(0)} \oplus \mathbf{6}^{(2)}
$$

which arises from the branching $\mathrm{SO}(4,4) \supset \mathrm{SO}(1,1) \times \mathrm{SO}(3,3)$. The stabilizer in $\mathrm{SO}(3,3)$ of a non-zero vector $\mathbf{e} \in \mathbf{6}^{(2)}$ is $\mathrm{SO}(3,2)$ if $\|\mathbf{e}\|^{2}>0$ for the signature $(3,3)$ quadratic norm, $\mathrm{SO}(2,3)$ if $\|\mathbf{e}\|^{2}<0$, or $\mathrm{SO}(2,2) \ltimes \mathbb{R}^{4}$ if $\|\mathbf{e}\|^{2}=0$. The last case returns to the minimal orbit discussed above, while the first two cases are related by parity. Thus, the 12-dimensional nilpotent orbits $[(+-+)]$ and $[(-+-)]$ are isomorphic to

$$
\mathcal{O}_{12}=\frac{S O_{0}(4,4)}{\mathrm{SO}(2,3, \mathbb{R}) \ltimes\left[(\mathbf{5} \oplus \mathbf{1})^{(2)}\right]} .
$$

On the other hand, the Young tableau $\left[(+-)^{4}\right]$ corresponds to the isomorphic 3-grading

$$
\mathfrak{s o}(4,4) \cong \boldsymbol{6}^{(-2)} \oplus\left(\mathfrak{g l}_{1} \oplus \mathfrak{s l}_{4}\right)^{(0)} \oplus \mathbf{6}^{(2)}
$$

associated to the branching $\mathrm{SO}(4,4) \supset \mathrm{SO}(1,1) \times \mathrm{SL}(4, \mathbb{R})$. The tableau $\left[(+-)^{4}\right]$ is "very even", therefore corresponds to 4 different orbits of $S O_{0}(4,4)$ (but a single orbit of $O(4,4)$ ). The stabilizer in $\mathrm{SL}(4, \mathbb{R})$ of a generic element $\mathbf{e} \in \mathbf{6}^{(2)}$ is $S p(4, \mathbb{R})$, therefore the 12dimensional nilpotent orbits $\left[(+-)^{4}\right]$ are isomorphic to

$$
\mathcal{O}_{12^{\prime}}=\frac{S O_{0}(4,4)}{\operatorname{Sp}(4, \mathbb{R}) \ltimes\left[(\mathbf{5} \oplus \mathbf{1})^{(2)}\right]} .
$$

Like the minimal orbit, the dimension 12 nilpotent orbits satisfy $A^{3}=0$, but have a higher degree of nilpotency in the 8-dimensional representations. For this purpose, it is useful to note that the unsigned Young tableaux $[(3)],\left[(2)^{4}\right],\left[(2)^{4}\right]$ are exchanged by triality. Thus, we conclude that $V^{2}=S^{3}=C^{2}=0$ for $\left[(+-)^{4}\right]_{I, I}$ and $\left[(+-)^{4}\right]_{I, I I}, V^{2}=S^{2}=C^{3}=0$ for $\left[(+-)^{4}\right]_{I I, I}$ and $\left[(+-)^{4}\right]_{I I, I I}$, and $S^{2}=V^{3}=C^{2}=0$ for both $[(+-+)]$ and $[(-+-)]$. Moreover, $\left[(+-)^{4}\right]_{I, I},\left[(+-)^{4}\right]_{I I, I}$ and $[(+-+)]$ are permuted amongst each other under triality, while $\left[(+-)^{4}\right]_{I, I I},\left[(+-)^{4}\right]_{I I, I I}$ and $[(-+-)]$ form a second triality orbit. The distinction between the two triplets of orbits corresponds to choosing the representative in the vector representation of $\mathrm{SO}(3,3)$ to satisfy either $\|\mathbf{e}\|^{2}>0$ or $\|\mathbf{e}\|^{2}<0$. 
The next level of nilpotent orbits, of real dimension 16, corresponds to two signed Young tableaux $\left[(+-+)(+-)^{2}\right]$ and $\left[(-+-)(+-)^{2}\right]$. They correspond to the 7 -grading

$$
\begin{aligned}
\mathfrak{s o}(4,4) \cong & \mathbf{2}^{(-3)} \oplus\left(\mathbf{1}_{(-2,0)} \oplus \mathbf{1}_{(1,-1)} \oplus \mathbf{1}_{(1,1)}\right)^{(-2)} \oplus\left(\mathbf{2}_{(2,0)} \oplus \mathbf{2}_{(-1,1)} \oplus \mathbf{2}_{(-1,-1)}\right)^{(-1)} \\
& \oplus\left(\mathfrak{g l}_{1} \oplus \mathfrak{g l}_{1} \oplus \mathfrak{g l}_{1} \oplus \mathfrak{s l}_{2}\right)^{(0)} \oplus\left(\mathbf{2}_{(-2,0)} \oplus \mathbf{2}_{(1,-1)} \oplus \mathbf{2}_{(1,1)}\right)^{(1)} \\
& \oplus\left(\mathbf{1}_{(2,0)} \oplus \mathbf{1}_{(-1,1)} \oplus \mathbf{1}_{(-1,-1)}\right)^{(2)} \oplus \mathbf{2}^{(3)}
\end{aligned}
$$

where 2 denotes the two-dimensional representation of $\mathfrak{s l}_{2}$, and the subscripts the weights with respect to the two extra $\mathfrak{g l}_{1}$ subalgebras. This originates again from the branching $\mathrm{SO}(4,4) \supset[\mathrm{SL}(2)]^{4}$, with a suitable choice of the non-compact Cartan generator $\mathbf{h}$ inside $[\mathrm{SL}(2)]^{3}$. The stabilizer of a generic element $\mathbf{e}$ in the grade 2 component (i.e. which carries a non-zero element in the three components $\mathbf{1}_{(2,0)}, \mathbf{1}_{(-1,1)}$ and $\left.\mathbf{1}_{(-1,-1)}\right)$ is $\mathfrak{s l}_{2}{ }^{(0)} \oplus(2 \times \mathbf{2})^{(1)} \oplus$ $(3 \times \mathbf{1})^{(2)} \oplus \mathbf{2}^{(3)}$ therefore

$$
\mathcal{O}_{16}=\frac{S O_{0}(4,4)}{\mathrm{SL}(2, \mathbb{R}) \ltimes\left[(2 \times \mathbf{2})^{(1)} \oplus(3 \times \mathbf{1})^{(2)} \oplus \mathbf{2}^{(3)}\right]} .
$$

Due to the 7 -grading, elements in this orbit satisfy $A^{4}=0$; moreover, since the Young tableau $\left[(+-+)(+-)^{2}\right]$ is invariant under triality, they also satisfy $V^{3}=S^{3}=C^{3}=0$.

Finally, the nilpotent orbits of real dimension 18 correspond three signed Young tableaux, all associated to the same 5 -grading (rather, even 9-grading)

$$
\mathfrak{s o}(4,4) \cong \mathbf{1}^{(-4)} \oplus(\mathbf{2} \otimes \mathbf{4})^{(-2)} \oplus\left(\mathfrak{g l}_{1} \oplus \mathfrak{s l}_{2} \oplus \mathfrak{s o}(2,2)\right)^{(0)} \oplus(\mathbf{2} \otimes \mathbf{4})^{(2)} \oplus \mathbf{1}^{(4)},
$$

identical to (A.30) save for the normalization of the non-compact Cartan generator $\mathbf{h}$. In particular, $\mathbf{e} \in \mathfrak{g}^{(2)}$ now transforms as a doublet of vectors of $\mathrm{SO}(2,2, \mathbb{R})$. If the norm of the two vectors is of the same sign, the stabilizer in $\mathrm{SO}(2,2, \mathbb{R})$ is $\mathrm{SO}(2)$. The broken $\mathrm{SO}(2)$ can be combined with $\mathrm{SO}(2) \subset \mathrm{SL}(2)$, leading to

$$
\mathcal{O}_{18,+}=\frac{S O_{0}(4,4)}{\mathrm{SO}(2, \mathbb{R}) \times \mathrm{SO}(2, \mathbb{R}) \ltimes\left[(\mathbf{2} \oplus \mathbf{2} \otimes \mathbf{2})^{(2)} \oplus \mathbf{1}^{(4)}\right]}
$$

If instead the two vectors have norms of opposite sign, the stabilizer in $\mathrm{SO}(2,2, \mathbb{R})$ is $\mathrm{SO}(1,1)$, leading instead to

$$
\mathcal{O}_{18,-}=\frac{S O_{0}(4,4)}{\mathrm{SO}(1,1, \mathbb{R}) \times \mathrm{SO}(1,1, \mathbb{R}) \ltimes\left[(\mathbf{2} \oplus \mathbf{2} \otimes \mathbf{2})^{(2)} \oplus \mathbf{1}^{(4)}\right]}
$$

The second one is invariant under parity, and so should correspond to the Young tableau associated to $[(+-+)(-+-)]$. The two other signed Young tableaux label two different orbits of $S O_{0}(4,4)$ each, labelled I,II, which are identified under $O(4,4)$. All 5 nilpotent orbits of dimension 18 satisfy $A^{5}=0$ (due to the even 9-grading) and $V^{3}=S^{3}=C^{3}=0$ (since $V^{3}=0$, obviously, and the Young tableau is invariant under triality). The condition $S^{3}=0$ (or equivalently $C^{3}=0$ ) is the condition of interest for extremal black holes.

In fact, from the Hasse diagram of the complex nilpotent orbits (A.29), it follows that the 18 dimensional orbits covers all nilpotent elements such that $A^{5}=0$. Higher dimensional orbits correspond to elements with a higher order of nilpotency in the adjoint 
representation: from the Young tableaux in (A.29), it is easy to see that the three 20dimensional complex orbits satisfy $A^{7}=V^{5}=S^{4}=C^{4}=0$ and triality images thereof, that the 22-dimensional orbits satisfies $A^{7}=V^{5}=S^{5}=C^{5}=0$, and that the 24dimensional orbit satisfies $A^{11}=V^{7}=S^{7}=C^{7}=0$.

Let us briefly discuss the fate of these orbits under the two-step restriction

$$
S O_{0}(4,4) \supset S O_{0}(4,3) \supset G_{2(2)},
$$

which corresponds to the truncation of the $S T U$ model to the $S T^{2}$ and $S^{3}$ models. In the first step $S O_{0}(4,4) \supset S O_{0}(4,3)$, the orbits associated to $\left[(-+-)^{2}\right],\left[(-+-)(+-)^{2}\right]$, $\left[(+-)^{4}\right]$ are lost, leaving 3 orbits $\left[(+-+)^{2}\right]_{I, I I},[(+-+)(-+-)]$ of dimension 14 and degree 5,2 orbits $\left[(+-+)(+-)^{2}\right]_{I, I I}$ of dimension 12 and degree 4,2 orbits $[(+-+)],[(-+-)]$ of dimension 10 and degree 3 , and the minimal orbit $\left[(+-)^{2}\right]$ of dimension 8 . In the second step $S O_{0}(4,3) \supset G_{2(2)}$, the degree 5 orbits reduce to two inequivalent orbits of dimension 10 (coming from $\left[(+-+)^{2}\right]_{I, I I}$ and $[(-+-)(+-+)]$, respectively), the degree 4 orbits to a single orbit of dimension 8 , and the only remaining orbit of degree 3 is the minimal orbit of dimension 6 .

\section{B Solving the non-BPS diagonalization problem}

The fake superpotential for extremal non-BPS black holes is given by (2.78), where $\varrho$ is proportional to the component of the central charge in the parametrization (2.68). In this section, we explain how to compute $W$ from the $\mathrm{SU}(8)$ invariants $\rho_{0}, \rho_{1}, \rho_{2}, \rho_{3}$ and $\varphi$. The latter can be computed from the central charge $Z_{i j}$ as explained below (2.11).

\section{B.1 General case}

To compute $\varrho$, let us define four real positive variables $\mathrm{x}_{n}$ as

$$
\mathrm{x}_{n}=\frac{1}{2}\left\{\begin{array}{ll}
\cos 2 \alpha+\frac{\xi_{n}}{\varrho} & \text { for } n=1,2,3 \\
\cos 2 \alpha-\frac{\xi_{1}+\xi_{2}+\xi_{3}}{\varrho} & \text { for } n=0
\end{array},\right.
$$

such that $\sum \mathrm{x}_{m}=2 \cos 2 \alpha$. Here and below, summations or products over $m$ will always range over $0,1,2,3$. Using (2.71), one may rewrite (2.78) as

$$
W=\frac{1}{2}\left(\frac{-\diamond(Z)}{\cos ^{2}(2 \alpha) \cdot \prod \mathrm{x}_{m}}\right)^{1 / 4} .
$$

In order to determine the variables $\mathrm{x}_{n}$, we equate (2.11) and (2.68) (keeping in mind that the rotations $R_{i}{ }^{j}$ and $\tilde{R}_{i}{ }^{j}$ are distinct for the two decompositions), obtaining

$$
\rho_{n}{ }^{2}=W^{2}\left|\mathrm{x}_{n}-e^{2 i \alpha}\right|^{2}=W^{2}\left(\left(\mathrm{x}_{n}-\cos (2 \alpha)\right)^{2}+\sin ^{2}(2 \alpha)\right) .
$$

which gives the algebraic equation

$$
\mathrm{x}_{n}^{2}-2 \mathrm{x}_{n} \cos (2 \alpha)+\left(1-\frac{\rho_{n}{ }^{2}}{W^{2}}\right)=0 .
$$


The positive solution of this quadratic equation is

$$
\mathrm{x}_{n}=\cos (2 \alpha)-\sqrt{\left(\rho_{n} / W\right)^{2}-\sin ^{2}(2 \alpha)} .
$$

Using moreover (B.2), we obtain that $W$ and $\cos (2 \alpha)$ are determined by the two equations

$$
\begin{gathered}
\sum_{n} \sqrt{\left(\rho_{n} / W\right)^{2}-\sin ^{2}(2 \alpha)}=\cos ^{2}(2 \alpha) \\
\prod_{n}\left(\cos (2 \alpha)-\sqrt{\left(\rho_{n} / W\right)^{2}-\sin ^{2}(2 \alpha)}\right)=-\frac{\diamond(Z)}{16 \cos ^{2}(2 \alpha) W^{4}} .
\end{gathered}
$$

In order to reduce these equations, we denote by $\sigma_{n}(r)$ the elementary symmetric functions of $r_{n} \equiv \sqrt{\left(\rho_{n} / W\right)^{2}-\sin ^{2}(2 \alpha)}$,

$$
\sigma_{1}(r)=\sum r_{m}, \quad \sigma_{2}(r)=\sum_{m<n} r_{m} r_{n}, \quad \sigma_{3}(r)=\sum_{m<n<p} r_{m} r_{n} r_{p}, \quad \sigma_{4}(r)=\prod r_{m},
$$

and by $\Sigma(r)$ the elementary symmetric functions of $r_{n}^{2}$, i.e. $\Sigma_{i}\left(r_{n}\right)=\sigma_{i}\left(r_{n}^{2}\right)$. The two sets of symmetric functions are related by

$$
\Sigma_{1}=\sigma_{1}^{2}-2 \sigma_{2}, \quad \Sigma_{2}=\sigma_{2}^{2}+2 \sigma_{4}-2 \sigma_{1} \sigma_{3}, \quad \Sigma_{3}=\sigma_{3}^{2}-2 \sigma_{2} \sigma_{4}, \quad \Sigma_{4}=\sigma_{4}^{2} .
$$

This can be inverted by eliminating $\sigma_{1}$ and $\sigma_{3}$ between these equations, leading to a quartic equation in $\sigma_{2}$,

$$
\left(\sigma_{2}^{2}+2 \sqrt{\Sigma_{4}}-\Sigma_{2}\right)^{2}-4\left(\Sigma_{3}+2 \sigma_{2} \sqrt{\Sigma_{4}}\right)\left(\Sigma_{1}+2 \sigma_{2}\right)=0
$$

Now, since $\sigma_{1}(r)=2 \cos 2 \alpha$, the first equation in (B.5) implies that

$$
\sigma_{2}(r)=2-\frac{1}{2} \sum \frac{\rho_{m}^{2}}{W^{2}}
$$

The second equation in (B.7) then leads to

$$
2 \sigma_{3}(r) \cos 2 \alpha-\sigma_{4}(r)=2-3 \sin ^{4} 2 \alpha-\left(1-\frac{3}{2} \sin ^{2} 2 \alpha\right) \sum \frac{\rho_{m}^{2}}{W^{2}}+\frac{1}{8 W^{4}}\left(\sum \rho_{m}^{4}-2 \sum_{n>m} \rho_{n}^{2} \rho_{m}^{2}\right)
$$

Substituting back into (B.5), we obtain

$$
\sigma_{4}(r)=-\sin ^{4} 2 \alpha+\frac{1}{2} \sin ^{2} 2 \alpha \sum_{n} \frac{\rho_{n}{ }^{2}}{W^{2}}+\frac{1}{8 W^{4}}\left(\sum \rho_{m}{ }^{4}-2 \sum_{n>m} \rho_{n}{ }^{2} \rho_{m}{ }^{2}-\frac{\diamond}{\cos ^{2} 2 \alpha}\right) .
$$

Squaring both sides, we obtain a polynomial of degree two in $W^{2}$

$$
\begin{aligned}
& {\left[\diamond-\left(\sum \rho_{m}{ }^{4}-2 \sum_{n>m} \rho_{n}{ }^{2} \rho_{m}{ }^{2}\right) \cos ^{2} 2 \alpha\right]^{2}-8^{2} \cos ^{4} 2 \alpha \prod \rho_{m}{ }^{2}} \\
& -2 W^{2} \sin ^{2} 4 \alpha\left(\left(\sum \rho_{m}{ }^{2}\right) \diamond-\left(\sum \rho_{m}{ }^{6}-\sum_{n \neq m} \rho_{n}{ }^{4} \rho_{m}{ }^{2}+2 \sum_{m>n>p} \rho_{m}{ }^{2} \rho_{n}{ }^{2} \rho_{p}{ }^{2}\right) \cos ^{2} 2 \alpha\right) \\
& +4 W^{4} \diamond \sin ^{2} 2 \alpha \sin ^{2} 4 \alpha=0 \quad \text { B. } 1
\end{aligned}
$$


Substituting (B.11) inside the third equation in (B.7), we obtain a second polynomial, whose difference with the former gives a polynomial of degree two in $\cos ^{2} 2 \alpha$,

$$
\begin{aligned}
& {\left[\prod\left(1-\frac{\rho_{m}{ }^{2}}{W^{2}}\right)+\frac{\diamond}{2 W^{4}}\left(1-\frac{1}{4} \sum_{m} \frac{\rho_{m}{ }^{2}}{W^{2}}\right)\right] \cos ^{4} 2 \alpha} \\
& -\frac{\diamond}{8 W^{4}}\left(1-\frac{1}{2} \sum_{m} \frac{\rho_{m}{ }^{2}}{W^{2}}-\frac{1}{8} W^{4}\left(\sum_{m} \rho_{n}{ }^{4}-2 \sum_{n>m} \rho_{n}{ }^{2} \rho_{m}{ }^{2}\right)\right) \cos ^{2} 2 \alpha-\frac{3 \diamond^{2}}{16^{2} W^{8}}=0
\end{aligned}
$$

By eliminating $\cos 2 \alpha$ between the two equations, we obtain a polynomial equation of degree 6 in $W^{2}$, whose coefficients are symmetric polynomials in $\rho_{m}{ }^{2}$ and polynomial in $\diamond$. It is convenient to write it in terms of $\diamond$ and the elementary symmetric functions $\Sigma_{i}\left(\rho_{m}\right)$, since they can all be expressed in terms of the $\mathrm{SU}(8)$ invariant functions of the central charges $Z_{i j}$ using (2.14). Writing $\Sigma_{i} \equiv \Sigma_{i}\left(\rho_{m}\right)$ for brevity, we obtain

$$
\begin{aligned}
& 64 W^{12}\left[-\diamond^{3}+\left(3 \Sigma_{1}^{2}-8 \Sigma_{2}\right) \diamond^{2}+\left(-3 \Sigma_{1}^{4}+16 \Sigma_{2} \Sigma_{1}^{2}-16 \Sigma_{3} \Sigma_{1}-16 \Sigma_{2}^{2}+64 \Sigma_{4}\right) \diamond\right. \\
& \left.+\left(\Sigma_{1}^{3}-4 \Sigma_{2} \Sigma_{1}+8 \Sigma_{3}\right)^{2}\right] \\
& +16 W^{10}\left[3 \Sigma_{1} \diamond^{3}+\left(-9 \Sigma_{1}^{3}+20 \Sigma_{2} \Sigma_{1}+24 \Sigma_{3}\right) \diamond^{2}\right. \\
& +\left(9 \Sigma_{1}^{5}-40 \Sigma_{2} \Sigma_{1}^{3}+32 \Sigma_{3} \Sigma_{1}^{2}+16\left(\Sigma_{2}^{2}-20 \Sigma_{4}\right) \Sigma_{1}+128 \Sigma_{2} \Sigma_{3}\right) \diamond \\
& \left.-\left(\Sigma_{1}^{3}-4 \Sigma_{2} \Sigma_{1}+8 \Sigma_{3}\right)\left(3 \Sigma_{1}^{4}-8 \Sigma_{2} \Sigma_{1}^{2}+32 \Sigma_{3} \Sigma_{1}-16 \Sigma_{2}^{2}+64 \Sigma_{4}\right)\right] \\
& +W^{8}\left[-15 \Sigma_{1}^{8}+240 \Sigma_{2} \Sigma_{1}^{6}-128 \Sigma_{3} \Sigma_{1}^{5}+32\left(28 \Sigma_{4}-37 \Sigma_{2}^{2}\right) \Sigma_{1}^{4}+2048 \Sigma_{2} \Sigma_{3} \Sigma_{1}^{3}\right. \\
& +256\left(7 \Sigma_{2}^{3}-12 \Sigma_{2} \Sigma_{4}\right) \Sigma_{1}^{2}+2048 \Sigma_{3}\left(4 \Sigma_{4}-3 \Sigma_{2}^{2}\right) \Sigma_{1}-27 \diamond^{4}+96 \diamond^{3}\left(\Sigma_{1}^{2}-3 \Sigma_{2}\right) \\
& -2 \diamond^{2}\left(63 \Sigma_{1}^{4}-408 \Sigma_{2} \Sigma_{1}^{2}+384 \Sigma_{3} \Sigma_{1}+496 \Sigma_{2}^{2}-960 \Sigma_{4}\right) \\
& +256\left(\Sigma_{2}^{4}-8 \Sigma_{4} \Sigma_{2}^{2}+16 \Sigma_{3}^{2} \Sigma_{2}+16 \Sigma_{4}^{2}\right)+8 \diamond\left(9 \Sigma_{1}^{6}-96 \Sigma_{2} \Sigma_{1}^{4}+112 \Sigma_{3} \Sigma_{1}^{3}\right. \\
& \left.\left.+16\left(17 \Sigma_{2}^{2}+8 \Sigma_{4}\right) \Sigma_{1}^{2}-576 \Sigma_{2} \Sigma_{3} \Sigma_{1}-64\left(2 \Sigma_{2}^{3}-8 \Sigma_{4} \Sigma_{2}-3 \Sigma_{3}^{2}\right)\right)\right] \\
& +W^{6}\left[-\Sigma_{1}^{9}+32 \Sigma_{2} \Sigma_{1}^{7}-64 \Sigma_{3} \Sigma_{1}^{6}+32\left(4 \Sigma_{4}-9 \Sigma_{2}^{2}\right) \Sigma_{1}^{5}+640 \Sigma_{2} \Sigma_{3} \Sigma_{1}^{4}\right. \\
& +1024\left(\Sigma_{2}^{3}-2 \Sigma_{4} \Sigma_{2}-\Sigma_{3}^{2}\right) \Sigma_{1}^{3}+2048 \Sigma_{3}\left(\Sigma_{2}^{3}-\Sigma_{2}^{2} \Sigma_{1}^{2}-4 \Sigma_{4} \Sigma_{2}-2 \Sigma_{3}^{2}\right) \\
& -256\left(5 \Sigma_{2}^{4}-24 \Sigma_{4} \Sigma_{2}^{2}-16 \Sigma_{3}^{2} \Sigma_{2}+16 \Sigma_{4}^{2}\right) \Sigma_{1}+\diamond^{3}\left(\Sigma_{1}^{3}-36 \Sigma_{2} \Sigma_{1}+216 \Sigma_{3}\right) \\
& -\diamond^{2}\left(3 \Sigma_{1}^{5}-104 \Sigma_{2} \Sigma_{1}^{3}+336 \Sigma_{3} \Sigma_{1}^{2}+368 \Sigma_{2}^{2} \Sigma_{1}+960 \Sigma_{4} \Sigma_{1}-1728 \Sigma_{2} \Sigma_{3}\right) \\
& +\diamond\left(3 \Sigma_{1}^{7}-100 \Sigma_{2} \Sigma_{1}^{5}+184 \Sigma_{3} \Sigma_{1}^{4}+16\left(41 \Sigma_{2}^{2}-28 \Sigma_{4}\right) \Sigma_{1}^{3}-1728 \Sigma_{2} \Sigma_{3} \Sigma_{1}^{2}\right. \\
& \left.\left.+64\left(-19 \Sigma_{2}^{3}+12 \Sigma_{4} \Sigma_{2}+12 \Sigma_{3}^{2}\right) \Sigma_{1}+128 \Sigma_{3}\left(31 \Sigma_{2}^{2}-60 \Sigma_{4}\right)\right)\right]
\end{aligned}
$$




$$
\begin{gathered}
+W^{4}\left[\left(\Sigma_{2} \Sigma_{1}^{4}-36 \Sigma_{3} \Sigma_{1}^{3}-8\left(\Sigma_{2}^{2}-42 \Sigma_{4}\right) \Sigma_{1}^{2}+144 \Sigma_{2} \Sigma_{3} \Sigma_{1}+16\left(\Sigma_{2}^{3}-36 \Sigma_{4} \Sigma_{2}-27 \Sigma_{3}^{2}\right)\right) \diamond^{2}\right. \\
+2\left(3072 \Sigma_{4}^{2}-16\left(5 \Sigma_{1}^{4}-36 \Sigma_{2} \Sigma_{1}^{2}-24 \Sigma_{3} \Sigma_{1}+64 \Sigma_{2}^{2}\right) \Sigma_{4}\right. \\
\left.+\left(\Sigma_{1}^{2}-4 \Sigma_{2}\right)\left(-\Sigma_{2}\left(\Sigma_{1}^{2}-4 \Sigma_{2}\right)^{2}+26 \Sigma_{1} \Sigma_{3}\left(\Sigma_{1}^{2}-4 \Sigma_{2}\right)+288 \Sigma_{3}^{2}\right)\right) \diamond \\
+4096 \Sigma_{2} \Sigma_{4}^{2}+\left(\Sigma_{1}^{2}-4 \Sigma_{2}\right)^{2}\left(\Sigma_{2}\left(\Sigma_{1}^{2}-4 \Sigma_{2}\right)^{2}-16 \Sigma_{1} \Sigma_{3}\left(\Sigma_{1}^{2}-4 \Sigma_{2}\right)-128 \Sigma_{3}^{2}\right) \\
\left.+64\left(\left(\Sigma_{1}^{2}-2 \Sigma_{2}\right)\left(\Sigma_{1}^{2}-4 \Sigma_{2}\right)^{2}+32 \Sigma_{1} \Sigma_{3}\left(\Sigma_{1}^{2}-4 \Sigma_{2}\right)+192 \Sigma_{3}^{2}\right) \Sigma_{4}\right] \\
+W^{2}\left[\left(-\Sigma_{1}^{2}+\diamond+4 \Sigma_{2}\right) \Sigma_{3}\left(\Sigma_{1}^{2}-4 \Sigma_{2}\right)^{3}+8\left(\diamond\left(-5 \Sigma_{1}^{3}+20 \Sigma_{2} \Sigma_{1}-72 \Sigma_{3}\right)\right.\right. \\
\left.+2\left(\Sigma_{1}^{2}-4 \Sigma_{2}\right)\left(\Sigma_{1}^{3}-4 \Sigma_{2} \Sigma_{1}+16 \Sigma_{3}\right)\right) \Sigma_{4}\left(\Sigma_{1}^{2}-4 \Sigma_{2}\right) \\
+\left[\left(\Sigma_{1}^{2}-4 \Sigma_{2}\right)^{2}-64 \Sigma_{4}\right]^{2} \Sigma_{4}=0
\end{gathered}
$$

Although still complicated, this polynomial is much simpler than what could be expected on the basis of the resultant of two generic polynomial equations of the form (B.13) and (B.12) above, which would generically lead to a polynomial of degree 12 in $W^{2}$. In the next subsections, we shall see that the degree is further reduced at special loci in the parameter space of $\left(\varphi, \rho_{m}\right)$.

\section{B.2 Near $\varphi=\pi / 4$}

At the point $\varphi=\pi / 4$, it is straightforward if tedious to check that the polynomial (B.14) at $\varepsilon=0$ admits the six roots

$$
\begin{aligned}
& W_{0}^{2}=\frac{1}{4}\left(\rho_{0}+\rho_{1}+\rho_{2}+\rho_{3}\right)^{2}, \quad W_{1}^{2}=\frac{1}{4}\left(\rho_{0}+\rho_{1}-\rho_{2}-\rho_{3}\right)^{2}, \\
& W_{2}^{2}=\frac{1}{4}\left(\rho_{0}-\rho_{1}+\rho_{2}-\rho_{3}\right)^{2}, \quad W_{3}^{2}=\frac{1}{4}\left(\rho_{0}-\rho_{1}-\rho_{2}+\rho_{3}\right)^{2}, \\
& W_{ \pm}^{2}=-\frac{\rho_{0} \rho_{1} \rho_{2} \rho_{3} \oslash}{4\left(\rho_{0} \rho_{1}+\rho_{2} \rho_{3}\right)\left(\rho_{0} \rho_{2}+\rho_{1} \rho_{3}\right)\left(\rho_{0} \rho_{3}+\rho_{1} \rho_{2}\right)} .
\end{aligned}
$$

The physical root corresponds to $W_{0}$. Indeed, using (2.15), one easily checks that the polynomial (B.12) vanishes at $\alpha=0$ when $\varphi=\pi / 4$. Moreover, the quadratic equation (B.3) is solved by

$$
\mathrm{x}_{n}=1-\frac{\rho_{n}}{W}=1-\frac{2 \rho_{n}}{\rho_{0}+\rho_{1}+\rho_{2}+\rho_{3}},
$$

which satisfies $\mathrm{x}_{m}>0, \sum \mathrm{x}_{m}=2$ as it should. We conclude that at $\varphi=\pi / 4$, the fake superpotential reduces to

$$
W_{0}=\frac{1}{2}\left(\rho_{0}+\rho_{1}+\rho_{2}+\rho_{3}\right),
$$

in agreement with [13]. It is straightforward to compute deviations to (B.17) away from the locus $\varphi=\frac{\pi}{4}$, in Taylor series of $\varepsilon \equiv \cos ^{2} 2 \varphi$. 
For the physical solution $W_{0}$, we find

$$
W_{0}=\frac{1}{2} \sigma_{1}\left[1-\frac{2 \sigma_{4}}{\sigma_{1} \sigma_{3}-4 \sigma_{4}} \varepsilon+\frac{2 \sigma_{4}^{2}\left(\sigma_{1}^{4} \sigma_{4}-\sigma_{1}^{3} \sigma_{2} \sigma_{3}+4 \sigma_{1}^{2} \sigma_{3}^{2}-24 \sigma_{1} \sigma_{3} \sigma_{4}+48 \sigma_{4}^{2}\right)}{\left(\sigma_{1} \sigma_{3}-4 \sigma_{4}\right)^{4}} \varepsilon^{2}+\ldots\right],
$$

where $\sigma_{i} \equiv \sigma_{i}\left(\rho_{m}\right)$ are the elementary symmetric functions of $\rho_{n}$. More generally, the expansion of $W_{n}$ to any order involves polynomials in $\sigma_{i}$ multiplying increasing powers of of $\varepsilon \sigma_{4} /\left(\sigma_{1} \sigma_{3}-4 \sigma_{4}\right)^{3}$. In contrast, the perturbation of the degenerate branch $W_{ \pm}$involves a power series in $\sqrt{\varepsilon}$.

\section{B.3 At $\rho_{2}=\rho_{3}-S^{2} T$ model}

At the point $\rho_{2}=\rho_{3}$, irrespective of the value of $\varepsilon$, the discriminant factorizes into the square of a linear factor in $W^{2}$, with double root at

$$
W_{2,3}^{2}=\frac{\left(\rho_{o}^{2}-\rho_{1}^{2}\right)^{2}}{4\left(\rho_{0}^{2}+\rho_{1}^{2}+(2-4 \varepsilon) \rho_{0} \rho_{1}\right)},
$$

and a quartic polynomial in $W^{2}$,

$$
\begin{aligned}
\Sigma_{2}^{\prime}( & \left.\left(\Sigma_{1}^{\prime}-4 \rho_{2}^{2}\right)^{2}-4 \Sigma_{2}^{\prime}\right)^{2}+W^{2}\left[36 \diamond \Sigma_{2}^{\prime}\left(4 \rho_{2}^{2}-\Sigma_{1}^{\prime}\right)-\diamond\left(4 \rho_{2}^{2}-\Sigma_{1}^{\prime}\right)^{3}\right. \\
& \left.-\left(\left(\Sigma_{1}^{\prime}-4 \rho_{2}^{2}\right)^{2}-4 \Sigma_{2}^{\prime}\right)\left(4 \Sigma_{2}^{\prime}\left(8 \rho_{2}^{2}-5 \Sigma_{1}^{\prime}\right)+\Sigma_{1}^{\prime}\left(\Sigma_{1}^{\prime}-4 \rho_{2}^{2}\right)^{2}\right)\right] \\
+W^{4} & {\left[-27 \diamond^{2}+6 \diamond\left(16 \rho_{2}^{4}-32 \rho_{2}^{2} \Sigma_{1}^{\prime}+7 \Sigma_{1}^{\prime 2}-12 \Sigma_{2}^{\prime}\right)+8 \Sigma_{2}^{\prime}\left(16 \rho_{2}^{4}-40 \rho_{2}^{2} \Sigma_{1}^{\prime}+15 \Sigma_{1}^{\prime 2}\right)\right.} \\
& \left.+\left(\Sigma_{1}^{\prime}-4 \rho_{2}^{2}\right)^{2}\left(16 \rho_{2}^{4}+24 \rho_{2}^{2} \Sigma_{1}^{\prime}-15 \Sigma_{1}^{\prime 2}\right)+16 \Sigma_{2}^{\prime 2}\right] \\
+16 W^{6} & {\left[3 \diamond\left(2 \rho_{2}^{2}+\Sigma_{1}^{\prime}\right)-\left(2 \rho_{2}^{2}-\Sigma_{1}^{\prime}\right)\left(16 \rho_{2}^{4}-3 \Sigma_{1}^{\prime 2}-4 \Sigma_{2}^{\prime}\right)\right]-64 W^{8}\left[\diamond-\left(\Sigma_{1}^{\prime}-2 \rho_{2}^{2}\right)^{2}\right] }
\end{aligned}
$$

where we have denoted $\Sigma_{1}^{\prime}=\rho_{0}^{2}+\rho_{1}^{2}, \Sigma_{2}^{\prime}=\rho_{0}^{2} \rho_{1}^{2}$.

At $\varepsilon=0$, all roots become elementary and match on the roots in (B.16).

It should be noted that the degeneracy between $W_{2,3}$ is not lifted on the $\rho_{2}=\rho_{3}$ locus, where $W_{2,3}$ is given exactly by (B.19), and happens to be independent of $\rho_{2}$. As discussed in section 3 , the locus $\rho_{2}=\rho_{3}$ corresponds to the two-modulus model of $\mathcal{N}=2$ supergravity, with prepotential $F=-S T^{2}$. While the roots of the quartic polynomial (B.20) can be computed explicitely, their expression is unilluminating.

\section{B.4 At $\rho_{1}=\rho_{2}=\rho_{3}-S^{3}$ model}

At the point $\rho_{1}=\rho_{2}=\rho_{3}$, the quartic polynomial (B.20) further factorizes into a linear factor in $W^{2}$ vanishing at the same point (B.19), which now occurs with multiplicity 3 ,

$$
W_{1,2,3}^{2}=\frac{\left(\rho_{0}^{2}-\rho_{1}^{2}\right)^{2}}{4\left(\rho_{0}^{2}+\rho_{1}^{2}+(2-4 \varepsilon) \rho_{0} \rho_{1}\right)},
$$


times an irreducible cubic polynomial in $W^{2}$,

$$
\left(\rho_{0}^{3}-9 \rho_{0} \rho_{1}^{2}\right)^{2}-12\left(\rho_{0}^{4}+18(2 \varepsilon-1) \rho_{0} \rho_{1}^{3}-3 \rho_{0}^{2} \rho_{1}^{2}\right) W^{2}+48\left(\rho_{0}^{2}+3 \rho_{1}^{2}\right) W^{4}-64 W^{6}=0 .
$$

The roots of the cubic polynomial are given by

$$
\begin{aligned}
& W_{0}^{2}=\frac{\rho_{0}^{2}}{4}\left(3 x^{2}+1+3 D x+3 D^{-1}\left(x(x+1)^{2}-4 \varepsilon x^{2}\right)\right) \\
& W_{+}^{2}=\frac{\rho_{0}^{2}}{4}\left(3 x^{2}+1-3(-1)^{1 / 3} D x+3(-1)^{2 / 3} D^{-1}\left(x(x+1)^{2}-4 \varepsilon x^{2}\right)\right), \\
& W_{-}^{2}=\frac{\rho_{0}^{2}}{4}\left(3 x^{2}+1+3(-1)^{2 / 3} D x-3(-1)^{1 / 3} D^{-1}\left(x(x+1)^{2}-4 \varepsilon x^{2}\right)\right),
\end{aligned}
$$

where $x \equiv \rho_{1} / \rho_{0}$ and $D$ is the real quantity

$$
D \equiv\left((x+1)^{3}-2 \varepsilon\left(3 x^{2}+1\right)-2 \sqrt{\varepsilon(1-\varepsilon)\left(3 x^{4}+8 x^{3}(1-2 \varepsilon)+6 x^{2}-1\right)}\right)^{1 / 3} .
$$

At $\varepsilon=0, D=x+1$ so that the three roots become elementary,

$$
\begin{aligned}
W_{0}^{2} & =\frac{1}{4}\left(\rho_{0}+3 \rho_{1}\right)^{2}-\frac{\varepsilon \rho_{0} \rho_{1}\left(\rho_{0}+3 \rho_{1}\right)^{2}}{3\left(\rho_{0}+\rho_{1}\right)^{2}}+O\left(\varepsilon^{2}\right), \\
W_{1,2,3}^{2} & =\frac{1}{4}\left(\rho_{0}-\rho_{1}\right)^{2}+\frac{\varepsilon \rho_{0} \rho_{1}\left(\rho_{0}-\rho_{1}\right)^{2}}{\left(\rho_{0}+\rho_{1}\right)^{2}}+O(\varepsilon), \\
W_{ \pm}^{2} & =\frac{1}{4} \rho_{0}\left(\rho_{0}-3 \rho_{1}\right) \pm \frac{i \rho_{0} \rho_{1} \sqrt{\varepsilon}}{2} \sqrt{9-\frac{12 \rho_{0}}{\rho_{0}+\rho_{1}}}+O(\varepsilon) .
\end{aligned}
$$

As the notation suggests, the roots $W_{1,2,3}$ match on to (B.16) as $\varepsilon=0$, while the roots $W_{0,1,2,3}$ match onto the roots of the quartic polynomial (B.20) at $\rho_{1}=\rho_{2}=\rho_{3}$. Using

$$
\varepsilon=\frac{1}{2}+\frac{\operatorname{Pfaff}(Z)+\operatorname{Pfaff}(\bar{Z})}{4 \rho_{0} \rho_{1} \rho_{2} \rho_{3}}=\frac{1}{2}+\frac{Z_{0} \bar{Z}_{1}^{3}+\bar{Z}_{0} Z_{1}^{3}}{4\left|Z_{0} Z_{1}^{3}\right|}
$$

one may rewrite the physical root as

$$
W_{0}=\frac{1}{2} \sqrt{\left|Z_{0}\right|^{2}+3\left(\left|Z_{1}\right|^{2}+L_{+}+L_{-}\right)},
$$

where $L_{+}$and $L_{-} \equiv\left|Z_{0} Z_{1}\right| D$ are given by

$$
L_{ \pm}^{3}=\left|Z_{1}\right|^{4}\left(\left|Z_{1}\right|^{2}+3|Z|^{2}\right)-\frac{1}{2}\left(Z \bar{Z}_{1}^{3}+\bar{Z} Z_{1}^{3}\right)\left(|Z|^{2}+3\left|Z_{1}\right|^{2}\right) \pm \frac{\sqrt{-\oslash}}{2}\left|Z \bar{Z}_{1}^{3}-\bar{Z} Z_{1}^{3}\right|
$$

As discussed in section 3, this expression provides the fake superpotential for non-BPS extremal black holes in the one-modulus model of $\mathcal{N}=2$ supergravity, with prepotential $F=-S^{3}$. 


\section{B.5 Near $\varphi=0$}

At $\varphi=0$, i.e. $\varepsilon=1$, the degree 6 polynomial (B.14) again becomes solvable, with roots at

$$
\begin{aligned}
& W_{ \pm}^{2}=\frac{\rho_{0} \rho_{1} \rho_{2} \rho_{3} \triangleright}{4\left(\rho_{1} \rho_{2}-\rho_{0} \rho_{3}\right)\left(\rho_{1} \rho_{3}-\rho_{0} \rho_{2}\right)\left(\rho_{0} \rho_{1}-\rho_{2} \rho_{3}\right)} \\
& W_{0}^{2}=\frac{1}{4}\left(\rho_{0}-\rho_{1}-\rho_{2}-\rho_{3}\right)^{2}, \quad W_{1}^{2}=\frac{1}{4}\left(\rho_{0}-\rho_{1}+\rho_{2}+\rho_{3}\right)^{2}, \\
& W_{2}^{2}=\frac{1}{4}\left(\rho_{0}+\rho_{1}-\rho_{2}+\rho_{3}\right)^{2}, \quad W_{3}^{2}=\frac{1}{4}\left(\rho_{0}+\rho_{1}+\rho_{2}-\rho_{3}\right)^{2} .
\end{aligned}
$$

Using again (2.15) and (B.12), one checks that the corresponding value of $\alpha$ is $\alpha=0$, the same value encountered at $\varphi=\pi / 4$. The quadratic equation (B.3) gives again $\mathrm{x}_{n}=$ $1-\left(\rho_{n} / W\right)$ so that the physical condition $\mathrm{x}_{n}$ now selects amongst $W_{0,1,2,3}$ the root

$$
\varphi=0: \quad W=\frac{1}{2}\left(\rho_{0}+\rho_{1}+\rho_{2}+\rho_{3}\right)-\min \left(\varrho_{m}\right) .
$$

For example, in the case of the canonical $\mathcal{N}=8$ ordering $\rho_{0} \geq \rho_{1} \geq \rho_{2} \geq \rho_{3}$, the physical root at $\varphi=0$ is $W_{3}$, while in the case of the $S^{3}$ model the physical root at $\varphi=0$ is still $W_{0}$.

Restricting to the locus $\rho_{2}=\rho_{3}$, the expansion near $\varepsilon=1$ is given by

$$
\begin{aligned}
W_{0}^{2}= & \frac{1}{4}\left(\rho_{0}-\rho_{1}-2 \rho_{2}\right)^{2}-\frac{\rho_{0} \rho_{1} \rho_{2}\left(-\rho_{0}+\rho_{1}+2 \rho_{2}\right)^{2}}{\left(\rho_{0}-\rho_{1}\right)\left(\rho_{0}\left(2 \rho_{1}+\rho_{2}\right)-\rho_{2}\left(\rho_{1}+2 \rho_{2}\right)\right)}(\varepsilon-1)+\ldots, \\
W_{1}^{2}= & \frac{1}{4}\left(\rho_{0}-\rho_{1}+2 \rho_{2}\right)^{2}+\frac{\rho_{0} \rho_{1} \rho_{2}\left(\rho_{0}-\rho_{1}+2 \rho_{2}\right)^{2}}{\left(\rho_{0}-\rho_{1}\right)\left(\rho_{0}\left(2 \rho_{1}-\rho_{2}\right)+\rho_{2}\left(\rho_{1}-2 \rho_{2}\right)\right)}(\varepsilon-1)+\ldots, \\
W_{2,3}^{2}= & \frac{1}{4}\left(\rho_{0}+\rho_{1}\right)^{2}+\frac{\rho_{0} \rho_{1}\left(\rho_{0}+\rho_{1}\right)^{2}}{\left(\rho_{0}-\rho_{1}\right)^{2}}(\varepsilon-1)+\ldots, \\
W_{ \pm}^{2}= & \frac{\rho_{0} \rho_{1}\left(\left(\rho_{0}+\rho_{1}\right)^{2}-4 \rho_{2}^{2}\right)}{4 \rho_{0} \rho_{1}-4 \rho_{2}^{2}} \\
& \mp \frac{\rho_{0} \rho_{1} \rho_{2}}{2\left(\rho_{2}^{2}-\rho_{0} \rho_{1}\right)^{2}} \sqrt{(1-\varepsilon)\left(\left(\rho_{0}+\rho_{1}\right)^{2}-4 \rho_{2}^{2}\right)\left(4\left(\rho_{2}^{2}-\rho_{0} \rho_{1}\right)^{2}-\rho_{2}^{2}\left(\rho_{0}-\rho_{1}\right)^{2}\right)}+\ldots
\end{aligned}
$$

In order to identify the branches consistently with the previous labeling, we have taken advantage of the solutions (B.19), (B.23), which are valid at any $\varepsilon$.

\section{Explicit solutions}

\section{C.1 D0-D4, BPS}

A prototype of a BPS solution is given by the D0-D4 black hole

$$
\begin{aligned}
\phi & =-\frac{1}{2} \log \left(H_{0} H^{1} H^{2} H^{3} / 4\right), \\
S & =i \sqrt{\frac{H_{0} H^{1}}{H^{2} H^{3}}}, \quad T=i \sqrt{\frac{H_{0} H^{2}}{H^{3} H^{1}}}, \quad U=i \sqrt{\frac{H_{0} H^{3}}{H^{1} H^{2}}}, \\
\zeta^{0} & =\frac{\sqrt{2}}{H_{0}}, \quad \tilde{\zeta}_{i}=-\frac{\sqrt{2}}{H^{i}}, \quad H_{0}=\sqrt{2}+Q_{0} \rho, \quad H^{i}=\sqrt{2}+P^{I} \rho
\end{aligned}
$$


with $\tilde{\zeta}_{0}=\zeta^{i}=\sigma=0$.

The mass and BH entropy are given by

$$
2 G M=\frac{1}{2 \sqrt{2}}\left|Q_{0}+P^{1}+P^{2}+P^{3}\right|, \quad S_{\mathrm{BH}}=\sqrt{Q_{0} P^{1} P^{2} P^{3}}
$$

consistent with the BPS mass formula at $S=T=U=i$.

One can check that the Noether matrix is nilpotent of degree 3, with Jordan form $\left[3^{2}, 1^{2}\right]$. The $\mathrm{SO}(4,4)$ metric in the Jordan basis takes the form

$$
\left(\begin{array}{cccccccc}
-\frac{Q_{0}}{2 P^{1}} & 0 & 0 & 0 & 0 & 0 & 0 & 0 \\
0 & -\frac{P^{2}}{2 P^{3}} & 0 & 0 & 0 & 0 & 0 & 0 \\
0 & 0 & 0 & 0 & -\frac{P^{2} P^{3}}{2} & 0 & 0 & 0 \\
0 & 0 & 0 & \frac{P^{2} P^{3}}{2} & 0 & 0 & 0 & 0 \\
0 & 0 & -\frac{P^{2} P^{3}}{2} & 0 & 0 & 0 & 0 & 0 \\
0 & 0 & 0 & 0 & 0 & 0 & 0 & -\frac{P^{1} Q_{0}}{2} \\
0 & 0 & 0 & 0 & 0 & 0 & \frac{P^{1} Q_{0}}{2} & 0 \\
0 & 0 & 0 & 0 & 0 & -\frac{P^{1} Q_{0}}{2} & 0 & 0
\end{array}\right)
$$

Since $P^{i} Q_{0}>0$ for all $i$, the signature is $\left[(+-+)^{2}(-)^{2}\right]$, and so BPS black holes correspond to the nilpotent orbit $\left[(+-+)^{2}(-)^{2}\right]_{I}$.

\section{C.2 D0-D4, non-BPS $Z_{*}=0$}

A prototype of the non-BPS, $Z_{*}=0$ solution of type (b) is the $D 0-D 4$ solution

The simplest solution with these properties is

$$
\begin{aligned}
& \phi=-\frac{1}{2} \log \left(H_{0} H^{1} H^{2} H^{3} / 4\right), \\
& S=i \sqrt{\frac{H_{0} H^{1}}{H^{2} H^{3}}}, \quad T=i \sqrt{\frac{H_{0} H^{2}}{H^{3} H^{1}}}, \quad U=i \sqrt{\frac{H_{0} H^{3}}{H^{1} H^{2}}}, \\
& \zeta^{0}=\frac{\sqrt{2}}{H_{0}}, \quad \tilde{\zeta}_{1}=-\frac{\sqrt{2}}{H^{1}}, \quad \tilde{\zeta}_{2}=\frac{\sqrt{2}}{H^{2}}, \quad \tilde{\zeta}_{3}=\frac{\sqrt{2}}{H^{3}}, \\
& H_{0}=\sqrt{2}+Q_{0} \rho, H^{1}=\sqrt{2}+P^{1} \rho, H^{2}=\sqrt{2}-P^{2} \rho, \quad H^{3}=\sqrt{2}-P^{3} \rho
\end{aligned}
$$

with $\tilde{\zeta}_{0}=\zeta^{i}=\sigma=0$, and charges such that

$$
Q_{0}>0, \quad P^{1}>0, \quad P^{2}<0, \quad P_{3}<0 .
$$

The mass and entropy are given by

$$
2 G M=\frac{1}{\sqrt{2}}\left(P^{1}-P^{2}-P^{3}+Q_{0}\right), \quad S_{\mathrm{BH}}=2 \pi \sqrt{Q_{0} P^{1} P^{2} P^{3}}
$$

The Noether charge is still nilpotent of order 3 and with Jordan form $\left[3^{2}, 1^{2}\right]$. Moreover, in the Jordan basis the metric once again takes the form (C.5). Thus, it corresponds to the nilpotent orbit $\left[(+-+)^{2}(-)^{2}\right]_{I I}$. The other cases $(c, d)$ are obtained by permutations, and correspond to the nilpotent orbits $\left[(-+-)^{2}(+)^{2}\right]_{I, I I}$. 


\section{C.3 D0-D4, non-BPS $Z \neq 0$}

The simplest non-BPS, $Z \neq 0$ solution with these properties is $[12,16,17]$

$$
\begin{aligned}
& \phi=-\frac{1}{2} \log \left(-H_{0} H^{1} H^{2} H^{3} / 4\right), \\
& S=i \sqrt{-\frac{H_{0} H^{1}}{H^{2} H^{3}}}, \quad T=i \sqrt{-\frac{H_{0} H^{2}}{H^{3} H^{1}}}, \quad U=i \sqrt{-\frac{H_{0} H^{3}}{H^{1} H^{2}}}, \\
& \zeta^{0}=\frac{\sqrt{2}}{H_{0}}, \quad \tilde{\zeta}_{1}=-\frac{\sqrt{2}}{H^{1}}, \quad \tilde{\zeta}_{2}=-\frac{\sqrt{2}}{H^{2}}, \quad \tilde{\zeta}_{3}=-\frac{\sqrt{2}}{H^{3}}, \\
& H_{0}=-\sqrt{2}+Q_{0} \rho, H^{1}=\sqrt{2}+P^{1} \rho, \quad H^{2}=\sqrt{2}+P^{2} \rho, \quad H^{3}=\sqrt{2}+P^{3} \rho
\end{aligned}
$$

with $\tilde{\zeta}_{0}=\zeta^{i}=\sigma=0$, and for definiteness, we focus on case (e) above,

$$
Q_{0}<0, \quad P^{1}>0, \quad P^{2}>0, \quad P^{3}>0 .
$$

The mass and entropy are given by

$$
2 G M=\frac{1}{\sqrt{2}}\left(P^{1}+P^{2}+P^{3}-Q_{0}\right), \quad S_{\mathrm{BH}}=2 \pi \sqrt{-Q_{0} P^{1} P^{2} P^{3}}
$$

The scalar potential has two flat directions at the horizon, generated by the vectors $P_{i} \partial_{B^{i}}-$ $P_{j} \partial_{B^{j}}, i, j=1 \ldots 3$.

The Noether charge is still nilpotent of order 3 and with Jordan form $\left[3^{2}, 1^{2}\right]$. The $\mathrm{SO}(4,4)$ metric in the Jordan basis is still given by (C.5), but now has signature $[(+-$ $+),(-+-),+,-]$

Open Access. This article is distributed under the terms of the Creative Commons Attribution Noncommercial License which permits any noncommercial use, distribution, and reproduction in any medium, provided the original author(s) and source are credited.

\section{References}

[1] S. Ferrara, R. Kallosh and A. Strominger, $N=2$ extremal black holes, Phys. Rev. D 52 (1995) 5412 [hep-th/9508072] [SPIRES].

[2] S. Ferrara and R. Kallosh, Universality of supersymmetric attractors, Phys. Rev. D 54 (1996) 1525 [hep-th/9603090] [SPIRES].

[3] S. Ferrara, G.W. Gibbons and R. Kallosh, Black holes and critical points in moduli space, Nucl. Phys. B 500 (1997) 75 [hep-th/9702103] [SPIRES].

[4] A. Sen, Black hole entropy function and the attractor mechanism in higher derivative gravity, JHEP 09 (2005) 038 [hep-th/0506177] [SPIRES].

[5] K. Goldstein, N. Iizuka, R.P. Jena and S.P. Trivedi, Non-supersymmetric attractors, Phys. Rev. D 72 (2005) 124021 [hep-th/0507096] [SPIRES].

[6] A. Dabholkar, A. Sen and S.P. Trivedi, Black hole microstates and attractor without supersymmetry, JHEP 01 (2007) 096 [hep-th/0611143] [SPIRES].

[7] F. Denef, Supergravity flows and D-brane stability, JHEP 08 (2000) 050 [hep-th/0005049] [SPIRES]. 
[8] B. Bates and F. Denef, Exact solutions for supersymmetric stationary black hole composites, hep-th/0304094 [SPIRES].

[9] R.R. Khuri and T. Ortín, A non-supersymmetric dyonic extreme Reissner-Nordstrom black hole, Phys. Lett. B 373 (1996) 56 [hep-th/9512178] [SPIRES].

[10] T. Ortín, Extremality versus supersymmetry in stringy black holes, Phys. Lett. B 422 (1998) 93 [hep-th/9612142] [SPIRES].

[11] P.K. Tripathy and S.P. Trivedi, Non-supersymmetric attractors in string theory, JHEP 03 (2006) 022 [hep-th/0511117] [SPIRES].

[12] A. Ceresole and G. Dall'Agata, Flow equations for non-BPS extremal black holes, JHEP 03 (2007) 110 [hep-th/0702088] [SPIRES].

[13] L. Andrianopoli, R. D'Auria, E. Orazi and M. Trigiante, First order description of black holes in moduli space, JHEP 11 (2007) 032 [arXiv:0706.0712] [SPIRES].

[14] G. Lopes Cardoso, A. Ceresole, G. Dall'Agata, J.M. Oberreuter and J. Perz, First-order flow equations for extremal black holes in very special geometry, JHEP 10 (2007) 063 [arXiv: 0706.3373] [SPIRES].

[15] J. Perz, P. Smyth, T. Van Riet and B. Vercnocke, First-order flow equations for extremal and non-extremal black holes, JHEP 03 (2009) 150 [arXiv:0810.1528] [SPIRES].

[16] K. Hotta and T. Kubota, Exact solutions and the attractor mechanism in non-BPS black holes, Prog. Theor. Phys. 118 (2007) 969 [arXiv:0707.4554] [SPIRES].

[17] E.G. Gimon, F. Larsen and J. Simon, Black holes in supergravity: the non-BPS branch, JHEP 01 (2008) 040 [arXiv:0710.4967] [SPIRES].

[18] S. Bellucci, S. Ferrara, A. Marrani and A. Yeranyan, stu black holes unveiled, arXiv: 0807.3503 [SPIRES].

[19] E.G. Gimon, F. Larsen and J. Simon, Constituent model of extremal non-BPS black holes, JHEP 07 (2009) 052 [arXiv: 0903.0719] [SPIRES].

[20] D. Gaiotto, W.W. Li and M. Padi, Non-supersymmetric attractor flow in symmetric spaces, JHEP 12 (2007) 093 [arXiv:0710.1638] [SPIRES].

[21] M. Günaydin, A. Neitzke, B. Pioline and A. Waldron, BPS black holes, quantum attractor flows and automorphic forms, Phys. Rev. D 73 (2006) 084019 [hep-th/0512296] [SPIRES].

[22] M. Berkooz and B. Pioline, 5D black holes and non-linear $\sigma$-models, JHEP 05 (2008) 045 [arXiv:0802.1659] [SPIRES].

[23] Y. Michel, B. Pioline and C. Rousset, $\mathcal{N}=4$ BPS black holes and octonionic twistors, JHEP 11 (2008) 068 [arXiv: 0806.4563] [SPIRES].

[24] G. Bossard, H. Nicolai and K.S. Stelle, Universal BPS structure of stationary supergravity solutions, JHEP 07 (2009) 003 [arXiv: 0902.4438] [SPIRES].

[25] G. Bossard and H. Nicolai, Multi-black holes from nilpotent Lie algebra orbits, arXiv:0906.1987 [SPIRES].

[26] G. Bossard, The extremal black holes of $N=4$ supergravity from $\mathrm{SO}(8,2+n)$ nilpotent orbits, arXiv:0906.1988 [SPIRES].

[27] P. Breitenlohner, D. Maison and G.W. Gibbons, Four-dimensional black holes from Kaluza-Klein theories, Commun. Math. Phys. 120 (1988) 295 [SPIRES]. 
[28] G. Clement and D.V. Galtsov, Stationary BPS solutions to dilaton-axion gravity, Phys. Rev. D 54 (1996) 6136 [hep-th/9607043] [SPIRES].

[29] E. Bergshoeff, W. Chemissany, A. Ploegh, M. Trigiante and T. Van Riet, Generating geodesic flows and supergravity solutions, Nucl. Phys. B 812 (2009) 343 [arXiv:0806. 2310] [SPIRES].

[30] B. Pioline, Lectures on on black holes, topological strings and quantum attractors, Class. Quant. Grav. 23 (2006) S981 [hep-th/0607227] [SPIRES].

[31] S. Ferrara and S. Sabharwal, Quaternionic manifolds for type II superstring vacua of Calabi-Yau spaces, Nucl. Phys. B 332 (1990) 317 [SPIRES].

[32] G.W. Moore, Arithmetic and attractors, hep-th/9807087 [SPIRES].

[33] S. Bellucci, A. Marrani, E. Orazi and A. Shcherbakov, Attractors with vanishing central charge, Phys. Lett. B 655 (2007) 185 [arXiv:0707.2730] [SPIRES].

[34] L. Andrianopoli, R. D'Auria, E. Orazi and M. Trigiante, First order description of $D=4$ static black holes and the Hamilton-Jacobi equation, arXiv:0905.3938 [SPIRES].

[35] M. Günaydin, A. Neitzke, B. Pioline and A. Waldron, Quantum attractor flows, JHEP 09 (2007) 056 [arXiv: 0707.0267] [SPIRES].

[36] A. Neitzke, B. Pioline and S. Vandoren, Twistors and black holes, JHEP 04 (2007) 038 [hep-th/0701214] [SPIRES].

[37] M. Günaydin, A. Neitzke, O. Pavlyk and B. Pioline, Quasi-conformal actions, quaternionic discrete series and twistors: $\mathrm{SU}(2,1)$ and $G_{2}(2)$, Commun. Math. Phys. 283 (2008) 169 [arXiv:0707.1669] [SPIRES].

[38] S. Ferrara and M. Günaydin, Orbits of exceptional groups, duality and BPS states in string theory, Int. J. Mod. Phys. A 13 (1998) 2075 [hep-th/9708025] [SPIRES].

[39] S. Bellucci, S. Ferrara, M. Günaydin and A. Marrani, Charge orbits of symmetric special geometries and attractors, Int. J. Mod. Phys. A 21 (2006) 5043 [hep-th/0606209] [SPIRES].

[40] D. Collingwood and W. McGovern, Nilpotent orbits in semisimple Lie algebras, Van Nostrand Reinhold Mathematics Series, New York U.S.A. (1993).

[41] D.Ž. Đoković, Classification of nilpotent elements in simple exceptional real Lie algebras of inner type and description of their centralizers, J. of Algebra 112 (1988) 503.

[42] E. Cremmer and B. Julia, The SO(8) supergravity, Nucl. Phys. B 159 (1979) 141 [SPIRES].

[43] B. de Wit and H. Nicolai, $\mathcal{N}=8$ supergravity, Nucl. Phys. B 208 (1982) 323.

[44] D.Ž. Đoković, The closure diagram for nilpotent orbits of the split real form of $E_{8}, C E J M 4$ (2003) 573.

[45] R. Kallosh and B. Kol, $E_{7}$ symmetric area of the black hole horizon, Phys. Rev. D 53 (1996) 5344 [hep-th/9602014] [SPIRES].

[46] L. Andrianopoli, R. D'Auria, S. Ferrara, P. Fré and M. Trigiante, $E_{7}(7)$ duality, BPS black-hole evolution and fixed scalars, Nucl. Phys. B 509 (1998) 463 [hep-th/9707087] [SPIRES].

[47] S. Ferrara and R. Kallosh, On $N=8$ attractors, Phys. Rev. D 73 (2006) 125005 [hep-th/0603247] [SPIRES].

[48] S. Ferrara and A. Marrani, N=8 non-BPS attractors, fixed scalars and magic supergravities, Nucl. Phys. B 788 (2008) 63 [arXiv:0705.3866] [SPIRES]. 
[49] S. Ferrara and A. Marrani, On the moduli space of non-BPS attractors for $N=2$ symmetric manifolds, Phys. Lett. B 652 (2007) 111 [arXiv:0706.1667] [SPIRES].

[50] M. Günaydin, G. Sierra and P.K. Townsend, Exceptional supergravity theories and the MAGIC square, Phys. Lett. B 133 (1983) 72 [SPIRES].

[51] D.Ž. Đoković, The closure diagrams for nilpotent orbits of the real form E IX of $E_{8}$, Asian J. Math. 5 (2001) 561.

[52] D.Ž. Đoković, The closure diagrams for nilpotent orbits of the real forms E VI and E VII of $E_{7}$, Representation Theory 5 (2001) 17.

[53] D.Ž. Đoković, The closure diagrams for nilpotent orbits of real forms of $E_{6}, J$. Lie Theory 11 (2001) 381.

[54] D.Ž. Đoković, The closure diagrams for nilpotent orbits of real forms of $F_{4}$ and $G_{2}, J$. Lie Theory 10 (2000) 491.

[55] A. Ceresole, R. D'Auria and S. Ferrara, The symplectic structure of $N=2$ supergravity and its central extension, Nucl. Phys. Proc. Suppl. 46 (1996) 67 [hep-th/9509160] [SPIRES].

[56] D.Ž. Đoković, N. Lemire, J. Sekiguchi, The closure ordering of adjoint nilpotent orbits in $\mathfrak{s o}(p, q)$, Tohoku Mat. J. 53 (2001) 395.

[57] A. Ceresole, G. Dall'Agata, S. Ferrara and A. Yeranyan, First order flows for $N=2$ extremal black holes and duality invariants, Nucl. Phys. B 824 (2010) 239 [arXiv:0908.1110] [SPIRES]. 